\title{
Nonlocal wave turbulence in non-Abelian plasmas
}

\author{
Yacine Mehtar-Tani \\ Institute for Nuclear Theory, University of Washington, \\ Seattle, WA 98195-1550, USA
}

\begin{abstract}
We investigate driven wave turbulence in non-Abelian plasmas, in the framework of kinetic theory where both elastic and inelastic processes are considered in the small angle approximation. The gluon spectrum, that forms in the presence of a steady source, is shown to be controlled by nonlocal interactions in momentum space, in contrast to the universal Kolmogorov-Zakharov spectra. Assuming strongly nonlocal interactions, we show that inelastic processes are dominant in the IR and cause a thermal bath to form below the forcing scale, as a result of a detailed balance between radiation and absorption of soft gluons by the hard ones. Above the forcing scale, the inelastic collision term reduces to an inhomogeneous diffusion-like equation yielding a spectrum that spreads to the UV as $t^{1 / 2}$, similarly to elastic processes. Due to nonlocal interactions the non-universal turbulent spectrum is not steady and flattens when time goes on toward the thermal distribution. This analysis is complemented by numerical simulations, where we observe that in the explored time interval the spectral exponent of the nonlocal turbulent cascade is close to -2 in agreement with simulations of classical Yang-Mills equations.
\end{abstract}

Keywords: Perturbative QCD, Heavy Ion Collisions, Wave Turbulence, Thermalization

INT-PUB-16-040

\section{Introduction}

For more than a decade, the approach to thermal equilibrium of the quark gluon plasma in the early time dynamics of ultrarelativistic heavy 
ion collisions, has been thoroughly studied. While a lot of progress has been made in uncovering the relevant phenomena at play, such as plasma instabilities, turbulence, particle production, izotropization, etc, the exact mechanisms by which the system reaches an early hydrodynamical behavior observed in the data is still unclear and remains the objet of intense research (see Ref. [1, 2, 3, 4] for reviews).

In this context, it was recognized early that plasma instabilities, such as Chromo-Weibel instabilities [5, 6], may play a role in spurring the isotropization of the system in weakly coupled plasmas (see e.g. Refs. [7] and references therein). P. Arnold and G. D. Moore have shown in 2005 [8], in particular, by solving numerically the Vlasov equation coupled to the classical Yang-Mills equation, that energy carried by unstable magnetic modes, that develop due to the initial anisotropy of the momentum distribution of the relatively hard particles, grows exponentially in a first stage, similarly to Abelian plasmas, then linearly due to gauge field self-interaction. During this phase, higher modes are excited generating a direct turbulent energy cascade characterized by a fitted power spectrum $\omega^{-2}[8,9]$. They suggested, in a follow-up paper [10], that the observed quasi-steady spectrum may be due to diffusion of hard modes on the strong classical field related to the unstable modes. However, this heuristic parametric argument appears to be in contradiction with the fact that diffusion conserves particle number and not energy [9].

Indeed, A. H. Mueller and collaborators argue, in a paper that appeared shortly after, that in non-Abelian plasmas, interactions are nonlocal in momentum space forcing the system in contact with a steady source of energy to be in thermal equilibrium at late times [11], that is, the spectrum must scale as $\omega^{-1}$ which is also a fixed point of the diffusion equation, as we shall see.

Later, simulations of classical Yang-Mills equations of motion, for a system that undergoes longitudinal expansion, have shown no evidence for plasma instabilities [12], confirming the standard bottom-up thermalization scenario [13]. Nevertheless, the turbulent spectrum obtained by Arnold and Moore in the Hard-Loop approximation is of a fundamental interest and remains to be fully understood.

The present work is an attempt to clarify this issue in the framework of kinetic theory, where both elastic and inelastic processes are taken into account. A complete analytic treatment proves to be difficult, however, some insights can be gained by analyzing the kinetic equation under certain, well motivated, approximations. We find for instance that strongly nonlocal in- 
elastic interactions obey a diffusion equation with a source term that conserves energy, and hence, is different from standard diffusion in momentum space.

Besides the aforementioned works $[8,11]$, most studies on turbulence in non-Abelian plasma are numerical and focus on the so-called free (decaying) turbulence $[14,15,12,16]$, that sets the early stages of thermalization in heavy ion collisions. After a transient regime, the system evolves in a selfsimilar way until the occupation number drops below unity where quantum effects begin to play a role in the final stage of thermalization. On the other hand, in forced (steady) turbulence where the system is in contact with a steady source, a stationary state is expected to be reached.

Forced turbulence is a chaotic phenomenon that is characterized by the transport of conserved quantities such as energy, in open systems, with constant fluxes through a wide interval of scales, the so-called inertial range, connecting the scale where energy is injected into the system, the forcing scale $\omega_{f}$, and the scale where it is dissipated. In the seminal work by Kolmogorov and Obukhov, known as KO41 theory, the famous 5/3 power law was derived for the energy spectrum of a fluid in a turbulent state $[17,18]$ under the assumption of scale invariance. This approximation is valid only if the relevant interactions are local, that is, confined to eddies of similar sizes, such that the physics in the inertial range is not sensitive to the injection and dissipation scales.

The phenomenon described above for classical fluids, occurs in a wide range of physical systems, in particular, in non-linear wave systems, such as surface water waves, atmospheric waves, waves in plasmas, non-linear optics and Bose-Einstein condensates for instance. In the early 1960's, V. E. Zakharov laid the foundation of the theory of (weak) wave turbulence theory by discovering that the kinetic equation that describes weakly nonlinear waves admits non-thermal stationary solutions in the form of power spectra associated with a constant flux for each integral of motion of the system $[17,18]$. Later, it was shown that the Boltzmann equation also admits similar power law solutions [19].

In the high occupancy limit, non-abelian plasmas described by classical Yang-Mills equations fall into the class of non-linear wave systems whose statistics can be studied in the framework of weak turbulence theory. The quantity of interest is the wave occupation number, $n_{k} \equiv n(\boldsymbol{k}, t)$ which is related to the equal time two-point correlation function $\left\langle a_{\boldsymbol{k}}(t) a_{\boldsymbol{k}^{\prime}}^{*}(t)\right\rangle \equiv$ 
$n(k, t) \delta\left(\boldsymbol{k}-\boldsymbol{k}^{\prime}\right)^{1}$, where $a_{k}$ is the classical analog of the gluon creation operator and the angle brackets $\langle\ldots\rangle$ stands for ensemble average with respect to the statistics of the forcing in driven(forced) turbulence or the initial condition for decaying turbulence. In the regime of weak non-linearity, that is when the coupling constant $g \ll 1$ for Yang-Mills theory, the occupation number obeys a kinetic equation of the form ${ }^{2}$,

$$
\frac{\partial n_{k}}{\partial t}=I_{k}[n],
$$

where the collision integral scales as $I_{k}[n] \sim n^{N-1}$ for dominant $N$ particle interactions (for instance $N=3$ for elastic 2 to 2 elastic scatterings). In deriving the wave kinetic equation (1.1) one averages over random wave phases which implies a Gaussian statistics and allows to express higher order correlation functions as a product of two-point correlation functions, yielding a closed equation for $n_{k}$.

As alluded to above, the purpose of this work is to study wave turbulence in non-Abelian plasmas, with the aim of better understanding the dynamics underlying the turbulent cascade resulting from Chromo-Weibel instabilities [8]. Our setup is similar to that of Ref. [11]: We consider an open system of gluons in contact with an isotropic and spectrally narrow steady source in order to mimic the growth of the unstable modes. Although instabilities are generated in anisotropic system, we assume that in the inertial range away from the source the system is statistically isotropic. Furthermore, we shall proceed in the framework of the effective kinetic theory proposed in Refs. [13, 21], that accounts for 2 to 2 elastic scattering and collinear effective 1 to 2 gluon branchings induced by small angle multiple scattering, that are parametrically of the same order (see discussion below). There are a few caveats that are as follows:

1. First, we restrict our analysis to the purely gluonic case and we assume the medium to be homogeneous and isotropic. It follows that the gluon occupation number is only function of the modulus of the wave number, i.e., $|\boldsymbol{k}| \equiv k$,

$$
(2 \pi)^{3} \frac{\mathrm{d} N}{\mathrm{~d}^{3} \boldsymbol{x} \mathrm{d}^{3} \boldsymbol{k}} \equiv n(\boldsymbol{k}, \boldsymbol{x}) \equiv n(k),
$$

\footnotetext{
${ }^{1}$ Here, color and polarization of the gluon field is omitted.

${ }^{2}$ For a recent derivation of the kinetic equation (with only 2 to 2 elastic processes considered) see Ref. [20]
} 
where $N$ is the number of gluons of a given spin and color.

2. In addition, we assume that the gluon momentum is much larger than its thermal mass, i.e., $k \gg m$ in order to use the homogeneous dispersion relation

$$
\omega(\boldsymbol{k}) \equiv|\boldsymbol{k}|
$$

and apply the small angle scattering approximation to the kinetic equations. In effect, gluon-gluon Coulomb scattering is dominated by small momentum transfer of the order of the Debye mass $m_{D} \sim m \ll k$, where $[22,21]$

$$
m_{D}^{2}=2 m^{2} \equiv \frac{g^{2} N_{c}}{\pi^{2}} \int \mathrm{d} k k^{2} \frac{\partial n(k)}{\partial k} .
$$

for Yang-Mills plasma.

3. We shall work in the classical limit of weakly non-linear waves where a kinetic description applies, namely,

$$
1 \ll n(k) \ll \frac{1}{g^{2}},
$$

with $g \ll 1$, the Yang-Mills coupling constant in a perturbative regime.

4. Finally, we consider the standard setup in the study of developed turbulence consisting in an open system in contact with an external source that injects gluons at a forcing frequency $\omega_{f}$, and sinks, that dissipate energy in the ultraviolet and the infrared at $\omega_{+}$and $\omega_{-}$, respectively, with a wide scale separation: between the source and the sinks, $\omega_{-} \ll \omega_{f} \ll \omega_{+}$.

In general, at leading order in the weak coupling $g$, one expects the dominant process to be 2 to 2 elastic scattering (four wave interaction) whose matrix element squaredis of order $g^{4}$. The corresponding kinetic equation admits two integrals of motion: energy and particle number ${ }^{3}$. Therefore, two nonthermal stationary spectra might form in the presence of a steady source. A direct energy cascade, characterized by a constant energy flux density, $P$, and an inverse particle cascade carrying a particle flux density $Q$.

\footnotetext{
${ }^{3}$ In general, momentum is also conserved but in an isotropic system it averages to zero.
} 
Owing to the fact that the Yang-Mills coupling is dimensionless and the dispersion relation is linear, there is no other dimensionfull quantity besides the frequency of the measured gluon, $\omega$. Hence, for a scale invariant system the collision integral scales parametrically as

$$
\dot{n}(\omega) \equiv I^{2 \rightarrow 2}[n] \sim g^{4} \omega n^{3},
$$

with $n \equiv n(\omega)$. On the other hand, the power spectra that carry constant flux of energy and constant flux of particles, respectively, verify

$$
P \equiv \dot{E} \sim \omega^{4} \dot{n} \equiv \text { const. and } Q \equiv \dot{N} \sim \omega^{3} \dot{n} \equiv \text { const. }
$$

Now, using Eq. (1.6) in Eq. (1.7) and solving for $n(\omega)$ as a function of constant energy flux $P$, and similarly for constant particle flux $Q$, we readily obtain the Kolmogorov-Zakharov (KZ) spectra associated with the direct energy cascade and inverse particle cascade, respectively,

$$
n(\omega) \sim P^{1 / 3} \omega^{-5 / 3} \text { and } n(\omega) \sim Q^{1 / 3} \omega^{-4 / 3} .
$$

The direction of the fluxes can be determined by the following simple argument: in a steady state, the energy and particle number pumping rate at $\omega_{f}$ is exactly balanced by the rate at which they are removed at the sinks, located at $\omega_{+}$and $\omega_{-}$, therefore, we must have $\dot{E}_{f}=\omega_{f} \dot{N}_{f}=\omega_{-} \dot{N}_{-}+\omega_{+} \dot{N}_{+}$ and $\dot{N}_{f}=\dot{N}_{-}+\dot{N}_{+}$. Now, solving for $\dot{N}_{+}$and $\dot{N}_{-}$in the limit $\omega_{-} \ll \omega_{f} \ll \omega_{+}$ we find that, $\dot{N}_{-} \simeq \dot{N}_{f}$ and $\dot{E}_{+}=\omega_{+} \dot{N}_{+} \simeq \dot{E}_{f}$, that means that particle number must be dissipated at $\omega_{-}$and energy at $\omega_{+}$.

The realization of the KZ spectra (1.8) depends upon two conditions:

1. Locality of interactions in momentum space, that is, the dynamics in the inertial range does not depend on the physics at the forcing or damping, besides the fluxes of the conserved quantities. This allows to use scale invariance and seek for power law solutions.

2. Suppression of inelastic processes (or more generally higher order processes) by extra powers of $g$, compared to elastic 2 to 2 scatterings.

However, while these conditions are satisfied for $\phi^{4}$ scalar theory (for instance) as we shall see in Section 2, neither of them is fulfilled by Yang-Mills equations. At weak coupling, higher order processes involve collinear divergences that are cutoff by thermal masses $m$, yielding a $m^{-2}$ pre-factor in the collision integral that breaks scale invariance. Moreover, since the thermal mass squared is proportional to $g^{2} n$, the supposedly suppressed 2 to 3 
process (of order $g^{6} n^{4}$ ) is enhanced in the region where two of the outgoing (or incoming) gluons are collinear (see Appendix E for a detailed demonstration). In turn, the collision integral for five wave interactions (2 to 3 scattering) is parametrically of the order of the four wave interaction term, that is, $I^{2 \rightarrow 3}[n] \sim g^{6} \omega^{3} m^{-2} n^{4} \sim g^{4} \omega n^{3}$, and thus it is a leading order effect $[13,21,23]$. In the quasi-collinear splitting approximation, the 2 to 3 inelastic process reduces to an effective 1 to 2 collinear splitting triggered by the exchange of a low momentum gluon (of the order $m_{D}$ ) in the $t$-channel that can be extended to processes that involve multiple scatterings acting coherently during the collinear branching process. Analogous approaches have been considered to cope with nonlocal collision integrals, for instance, in describing kelvin waves in superfluids [24] or interactions of hard modes with a Bose-Einstein condensate $[25,26]$, that cause the breakdown of the 4 wave interaction into an effective three wave interaction of acoustic type [27].

Let us now summarize our results. In Section 2, as a warm up exercise, we discuss $\phi^{4}$ scalar theory that admits local KZ spectra as non-thermal stationary states, in order to illustrate and highlight the differences with Yang-Mills theories. We compute in particular the non-universal KZ constant factors. In the following two sections, we turn to classical Yang-Mills theory where elastic and inelastic collision integrals are first discussed separately. In Section 3, 2 to 2 elastic processes are first analyzed. Because the dominant process is the small angle Coulomb scattering, the collision integral can be reduced to a Fokker-Planck (FP) equation which is solved in the stationary case. In the inertial range to the left of the forcing scale (away from the source and sinks), the steady state power spectrum is of the form $Q_{f}^{1 / 3} \omega_{f}^{-1 / 3} \omega^{-1}$. It is related to an inverse particle cascade. The explicit dependence on the forcing scale $\omega_{f}$ reflects the non universality of the stationary state. In addition, we find that although energy is conserved, there is no finite steady state associate with the direct energy cascade. It follows that above the forcing scale, where the direct cascade is expected to form, the spectrum vanishes in the limit of infinitely large inertial range, i.e., when energy is dissipated at infinity. Numerical simulations of the FP equation are also performed to investigate quantitatively the approach toward the steady state obtained analytically.

In Section 4, we turn to inelastic processes that can be described by effective quasi-collinear 1 to 2 scattering. This process conserves energy only. We recover the two KZ spectra $\omega^{-2}$ and $\omega^{-7 / 4}$ that correspond to the BetheHeitler $(\mathrm{BH})$, single scattering, and Landau-Pomeranchuk-Migdal (LPM) 
multiple scattering regimes, respectively, separated by the scale $\omega_{\mathrm{BH}} \sim \hat{q} \ell_{\mathrm{mfp}}^{2}$, where $\hat{q}$ is the momentum space diffusion coefficient and $\ell_{\mathrm{mfp}}$ is the mean free path. Following Zakharov's approach, we show that the latter spectrum is nonlocal and hence can not be realized, whereas the former yields a logarithmically divergent energy flux and thus is marginally nonlocal. This means that up to a mild logarithmic factor the $\mathrm{KZ}$ spectrum $\omega^{-2}$ is physically relevant. If follows that at least in the LPM regime strongly asymmetric gluon splittings are expected to be the dominant processes at late times. In this limit, the collision integral reduces to a inhomogeneous diffusion-like equation.

As a result, the turbulent spectrum forms in the wake of a wave front that scales as $t^{1 / 2}$ in the ultraviolet with a diffusion coefficient of the order of the elastic diffusion coefficient $\hat{q}$. In this diffusion process energy is directly transferred from the source to the hard gluons via soft gluon absorption. On the other hand, radiation is suppressed due to the rapid fall off of the spectrum as long as the spectrum is steeper than $\omega^{-1}$.

Because of the inhomogeneity of both the elastic and inelastic collision integrals in the diffusion approximation the resulting spectrum is not steady in the wake of the UV wave front and flattens as time goes on. A numerical simulation of the effective kinetic equation in the LPM regime shows the flattening of the spectrum, however the observed power exponents are closer to -2 than -1 .

Below the forcing scale, deep in the infrared regime, the dominant nonlocal processes are radiation and absorption of soft gluons by the source that balance each other due to the singular behavior of the radiation rate, forcing the soft sector to be in thermal equilibrium although the system is constantly driven out of equilibrium. This phenomenon is analyzed in detail in a recent work on thermalization of isotropic plasmas [28].

In Section 5, the interplay of elastic and inelastic processes is discussed. Numerical simulations of the complete collision integral, including elastic and inelastic processes, are performed, and we observe that the late time behavior is similar to that of inelastic alone, namely, the existence of a thermal bath below the forcing scale and a quasi-steady power spectrum close to $\omega^{-2}$ develops. As in the case of the inelastic scatterings alone, we argue that this spectrum corresponds to a long lived transient regime and that asymptotically a flattening of the spectrum toward the thermal one may occur, with a small deviation that would account for finite energy flux.

Finally, in the last section we summarize and conclude. 


\section{KZ steady state spectra in $\phi^{4}$ theory}

Before we proceed with the analysis of wave turbulence in Yang-Mills theory, let us first address the somewhat academic example of $\phi^{4}$ scalar theory, to demonstrate how the power spectra derived from dimensional analysis in the introduction are indeed stationary solutions of the kinetic equation. The latter is of the form of the Boltzmann-Nordheim (BN) equation, which describes the transport properties of a gas of bosons that undergo elastic 2 to 2 scattering [29]. It is widely used in the context of Bose-Einstein condensation (see Ref. [30] for instance).

Given the dispersion relation $\omega_{k} \equiv \sqrt{\boldsymbol{k}^{2}+m^{2}}$, where $m$ is the mass of the bosonic particles, the leading order contribution to the collision integral at small coupling (weak nonlinearity) is the 2 to 2 scattering process, even if there was a cubic interaction term in the Lagrangian. Indeed, the 3-wave resonance condition, that is, $\omega_{1}+\omega_{2}=\omega_{3}$ for $\boldsymbol{k}_{1}+\boldsymbol{k}_{2}=\boldsymbol{k}_{3}$ for 1 to 2 particle decay (and the inverse process), is only fulfilled when all momenta are collinear and hence, is kinematically suppressed. Likewise, the 1 to 3 and 0 to 4 particles decay vanish and only the 2 to 2 matrix element contributes to the collision integral that takes the standard form

$$
I^{2 \rightarrow 2}[n]=\frac{1}{2 !} \int_{123} \frac{1}{2 \omega_{k}}\left|M_{23 \rightarrow 1 k}\right|^{2} F[n](2 \pi)^{4} \delta\left(k+k_{1}-k_{2}-k_{3}\right),
$$

where $\left|M_{23 \rightarrow 1 k}\right|^{2}=g^{4}$ is the (squared) matrix element for the elastic process $k_{2}+k_{3} \rightarrow k+k_{1}$ as illustrated in Figure 1 for the interaction Lagrangian density $g^{2} \phi^{4} / 4$ !, and

$$
F[n]=n_{2} n_{3}\left(1+n_{k}\right)\left(1+n_{1}\right)-n_{k} n_{1}\left(1+n_{2}\right)\left(1+n_{3}\right),
$$

where $n_{i} \equiv n\left(\boldsymbol{k}_{i}\right)$. Internal degrees of freedom like spin are averaged over for the measured boson $(k)$ and summed over for the integrated ones $(1,2,3)$. The symmetry factor $1 / 2$ !, in Eq. (2.1), accounts for the exchange of the incoming (outgoing) particles 1 and 2. Also, we have used the shorthand notation

$$
\int_{i} \equiv \int \frac{\mathrm{d}^{3} k_{i}}{2(2 \pi)^{3} \omega_{i}}
$$

for the invariant measure. For isotropic media the kinetic equation admits two integrals of motion, energy and particle number, respectively,

$$
E=\int \frac{\mathrm{d}^{3} k}{(2 \pi)^{3}} \omega n_{k} \quad \text { and } \quad N=\int \frac{\mathrm{d}^{3} k}{(2 \pi)^{3}} n_{k},
$$




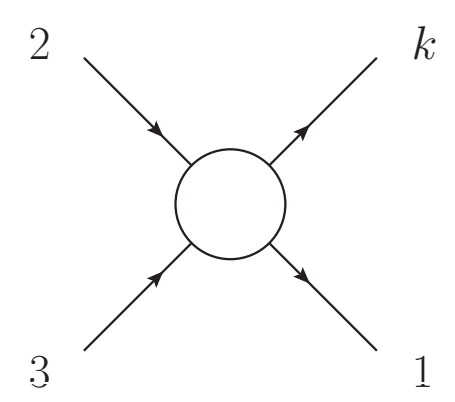

(a)

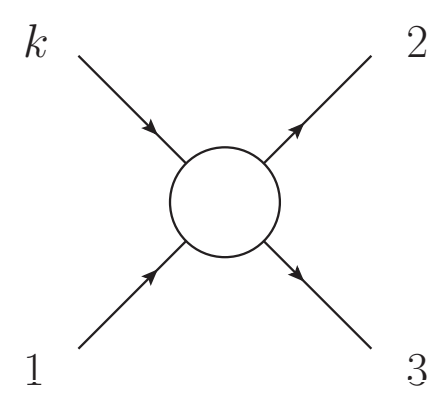

(b)

Figure 1: Illustration of the 2 to 2 scattering process. The two contributions of Eq. (2.2), corresponding to the gain (left) and loss (right) terms, are depicted.

where $\omega \equiv \omega_{k}$. Using the symmetries of the 2 to 2 matrix element under the exchange of bosons in the initial and final state, and under time reversal, $\left|M_{12 \rightarrow 3 k}\right|^{2}=\left|M_{21 \rightarrow 3 k}\right|^{2}=\left|M_{12 \rightarrow k 3}\right|^{2}=\left|M_{3 k \rightarrow 12}\right|^{2}$, and assuming that the integrant vanishes at infinite momenta such that there is no "leakage" of energy or particle number at infinity, it is easy to verify that $E$ and $N$ are conserved, that is,

$$
\dot{N}=\int \frac{\mathrm{d}^{3} k}{(2 \pi)^{3}} \frac{\partial n_{k}}{\partial t}=\int \frac{\mathrm{d}^{3} k}{(2 \pi)^{3}} I_{k}^{2 \rightarrow 2}[n]=0 .
$$

for the particle number, and

$$
\dot{E}=\int \frac{\mathrm{d}^{3} k}{(2 \pi)^{3}} \omega \frac{\partial n_{k}}{\partial t}=\int \frac{\mathrm{d}^{3} k}{(2 \pi)^{3}} \omega I_{k}^{2 \rightarrow 2}[n]=0
$$

for energy. Now, owing to the Boltzmann's $H$-theorem, an isolated system must evolve toward a state of maximum entropy, that corresponds to thermal equilibrium characterized by the Bose-Einstein (BE) distribution. At equilibrium, the collision integral vanishes $F[n]=0$ (as well as all fluxes), as a result of the detailed balance between the gain and loss terms in Eq. (2.2), independently of the form of the matrix element and in particular its degree of homogeneity, in contrast with the non-thermal fixed point. The occupation number is thus given by the BE distribution,

$$
n_{\mathrm{BE}}(k)=\frac{1}{\exp \left(\frac{\omega-\mu}{T}\right)-1},
$$


where $T$ is the equilibrium temperature and $\mu$ the chemical potential. In the regime in which $n \gg 1$ (classical wave regime), one can neglect the quadratic terms in $n$ and Eq. (2.2) becomes

$$
F[n] \simeq n_{2} n_{3} n_{k}+n_{2} n_{3} n_{1}-n_{1} n_{k} n_{2}-n_{1} n_{k} n_{3}
$$

In this case, the thermal fixed point reduces to the Rayleigh-Jeans distribution, that can be deduced from the BE distribution by taking the limit $\omega-\mu \ll T$,

$$
n_{\mathrm{RJ}}(k)=\frac{T}{\omega-\mu} .
$$

Let us turn now to the finite flux KZ solutions of Eq. (2.1) with $F[n]$ given by Eq. (2.8) ${ }^{4}$. For scale invariant media, besides the thermal fixed point (2.9), Eq. (2.1) admits non-thermal stationary power solutions, known as the Kolmogorov-Zakharov (KZ) spectra, associated with constant energy and particle fluxes. In general, KZ spectra are expected to form in open systems in contact with a source, which steadily injects energy into the systems, and sinks for dissipation.

For isotropic media $F[n]$ is independent of angles. Hence, one can integrate over the solid angle (see Appendix B for details) and the collision integral, Eq. (2.1), yields

$$
I^{2 \rightarrow 2}[n]=\frac{1}{\omega^{2}} \int \mathrm{d} \omega_{1} \mathrm{~d} \omega_{2} \mathrm{~d} \omega_{3} U\left(\omega, \omega_{1}, \omega_{2}, \omega_{3}\right) F[n] \delta\left(\omega+\omega_{1}-\omega_{2}-\omega_{3}\right),
$$

with

$$
U\left(\omega, \omega_{1}, \omega_{2}, \omega_{3}\right)=\frac{g^{4}}{2(4 \pi)^{3}} \min \left(\omega, \omega_{1}, \omega_{2}, \omega_{3}\right),
$$

where $\mathrm{d} \Omega_{i}(i=1,2,3)$ is the integration measure for the solid angle. Note that $U\left(\omega, \omega_{1}, \omega_{2}, \omega_{3}\right)$ possesses the same symmetries as $\left|M_{k 1 \rightarrow 23}\right|^{2}$, namely,

$$
U\left(\omega, \omega_{1}, \omega_{2}, \omega_{3}\right)=U\left(\omega_{1}, \omega, \omega_{2}, \omega_{3}\right)=U\left(\omega, \omega_{1}, \omega_{3}, \omega_{2}\right)=U\left(\omega_{2}, \omega_{3}, \omega, \omega_{1}\right) .
$$

\footnotetext{
${ }^{4}$ Note that scale invariant KZ spectra exist only for homogenous collision integrals.
} 
Integrating over $\omega_{1}$, Eq. (2.10) can be written as

$$
I^{2 \rightarrow 2}[n]=\frac{1}{\omega^{2}} \int_{\Delta} \mathrm{d} \omega_{2} \mathrm{~d} \omega_{3} U\left(\omega, \omega_{1}, \omega_{2}, \omega_{3}\right) F[n],
$$

where the integration domaine in the $\left(\omega_{2}, \omega_{3}\right)$ plane,

$$
\Delta \equiv\left\{\omega_{2}+\omega_{3}>\omega, \omega_{2}>0, \omega_{3}>0\right\},
$$

can be divided into four subregions (cf. Figure 2).

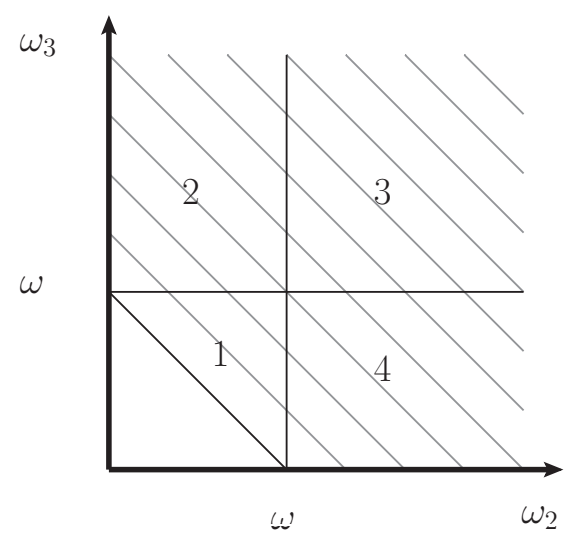

Figure 2: The integration regions for the integral given in Eq. (2.13) where the lower left triangle is excluded.

Now, let us look for power law solutions of the form

$$
n(\omega) \equiv A \omega^{x},
$$

where $A$ and $x$ are to be determined. As already discussed in the introduction, the spectra exponent is universal, and can be derived by dimensional analysis. The constant $A$, known as the $\mathrm{KZ}$ constant, depends however on the details of the collision integral. Using Eq. (2.15) in Eq. (2.13), the KZ spectra can be made manifest after performing the Zakharov conformal transformations (cf. Appendix A), that allow us to map all integration regions onto that where $\omega$ is the largest frequency of the quartet $\left(\omega_{1}, \omega_{2}, \omega_{3}, \omega\right)$. Thus, 
Eq. (2.10) yields

$$
\begin{aligned}
I^{2 \rightarrow 2}[n] & =\int_{0}^{\omega} \mathrm{d} \omega_{3} \int_{\omega-\omega_{2}}^{\omega} \mathrm{d} \omega_{2} U\left(\omega, \omega_{1}, \omega_{2}, \omega_{3}\right) \\
& {\left[1+\left(\frac{\omega_{1}}{\omega}\right)^{y}-\left(\frac{\omega_{2}}{\omega}\right)^{y}-\left(\frac{\omega_{3}}{\omega}\right)^{y}\right] F\left(\omega, \omega_{1}, \omega_{2}, \omega_{3}\right), }
\end{aligned}
$$

with $y=-3 x-5-\nu$, where $\nu=1$ is the degree of homogeneity of $U$ and

$$
F\left(\omega, \omega_{1}, \omega_{2}, \omega_{3}\right) \equiv A^{3} \omega^{x} \omega_{1}^{x} \omega_{2}^{x} \omega_{3}^{x}\left[\omega^{-x}+\omega_{1}^{-x}-\omega_{2}^{-x}-\omega_{3}^{-x}\right],
$$

We can readily extract the power exponent for which $I^{2 \rightarrow 2}[n]=0$. First, we recover the thermal fixed points that correspond to the two limits of the RJ distribution (2.9), $x=1$ and $x=0$. For $y=1$ or $y=0$, we obtain the $\mathrm{KZ}$ non-thermal fixed points, respectively,

$$
x=-\frac{5}{3} \quad \text { and } \quad x=-\frac{4}{3},
$$

that were infered by dimensional analysis in the introduction. We turn now to the determination of the non-universal constant $A$. Note that since $\min \left(\omega, \omega_{1}, \omega_{2}, \omega_{3}\right)=\omega_{1}$ in Eq. (2.16), we have $U \sim \omega_{1}$. Now, introducing the scaling variables: $z_{1}=\omega_{1} / \omega, z_{2}=\omega_{2} / \omega$ and $z_{3}=\omega_{3} / \omega$, Eq. (2.16) yields

$$
I^{2 \rightarrow 2}[n]=\frac{g^{4} A^{3}}{2(4 \pi)^{3}} f(x) \omega^{3 x+1},
$$

where the dimensionless function $f(x)$ is given by

$$
\begin{aligned}
f(x)= & \int_{0}^{1} \mathrm{~d} z_{2} \int_{1-z_{2}}^{1} \mathrm{~d} z_{3} z_{1}^{x+1} z_{2}^{x} z_{3}^{x} \\
& \times\left(1+z_{1}^{y}-z_{2}^{y}-z_{3}^{y}\right)\left(1+z_{1}^{-x}-z_{2}^{-x}-z_{3}^{-x}\right),
\end{aligned}
$$

with $z_{1}=z_{2}+z_{3}-1$ from energy conservation. It is straightforward to check that $f(x)$ converges on the KZ spectra (2.18) ensuring the locality of interactions for relativistic 2 to 2 scattering in $\phi^{4}$ theory. A more complete 
analysis would include the study of the stability of the KZ spectra under small perturbations, but this is beyond the scope of the present work.

Let us turn now to the fluxes associated with the KZ spectra. The energy and particle fluxes through a sphere of radius $\omega$ read, respectively,

$$
P(\omega) \equiv \int_{\omega_{0}<\omega} \frac{\mathrm{d}^{3} \boldsymbol{k}_{0}}{(2 \pi)^{3}} \omega_{0} \frac{\partial n\left(\omega_{0}\right)}{\partial t}=\frac{1}{2 \pi^{2}} \int_{0}^{\omega} \mathrm{d} \omega_{0} \omega_{0}^{3} I^{2 \rightarrow 2}[n]
$$

and

$$
Q(\omega) \equiv \int_{\omega_{0}<\omega} \frac{\mathrm{d}^{3} \boldsymbol{k}_{0}}{(2 \pi)^{3}} \frac{\partial n\left(\omega_{0}\right)}{\partial t}=\frac{1}{2 \pi^{2}} \int_{0}^{\omega} \mathrm{d} \omega_{0} \omega_{0}^{2} I^{2 \rightarrow 2}[n] .
$$

Inserting Eq. (2.19) in Eq. (2.21) and integrating over $\omega_{0}$ assuming $3 x+5>0$, one finds

$$
P(\omega)=-\frac{2 g^{4} A^{3}}{(4 \pi)^{5}} \frac{\omega^{3 x+5}}{3 x+5} f(x) .
$$

Evidently, the energy flux (2.23) diverges in the limit $x \rightarrow-5 / 3$, but at the same time $f(-5 / 3)=0$, yielding an undetermined form. Using the L'Hopital rule, we find that the limit is finite and reads,

$$
\begin{aligned}
& \lim _{x \rightarrow-5 / 3} \frac{f(x)}{3 x+5}=\frac{1}{3} \lim _{x \rightarrow-5 / 3} f^{\prime}(x)=\int_{0}^{1} \mathrm{~d} z_{2} \int_{1-z_{2}}^{1} \mathrm{~d} z_{3} z_{1}^{-2 / 3} z_{2}^{-5 / 3} z_{3}^{-5 / 3} \\
& \times\left(z_{1} \ln z_{1}-z_{2} \ln z_{2}-z_{3} \ln z_{3}\right)\left(1+z_{1}^{5 / 3}-z_{2}^{5 / 3}-z_{3}^{5 / 3}\right) \simeq 2.6
\end{aligned}
$$

It follows that the energy flux (2.23) is negative and independent of $\omega$. Therefore, it is directed toward increasing $\omega$ and thus, energy must dissipate in the ultraviolet. By solving Eq. (2.23) for the constant $A$ as a function of a constant flux of energy $P(\omega) \equiv-P_{f}<0$, we obtain the KZ constant associated with a direct energy cascade,

$$
n(\omega)=C_{\mathrm{KZ}} g^{-4 / 3} P_{f}^{1 / 3} \omega^{-5 / 3}, \quad \text { where } \quad C_{\mathrm{KZ}}=39.2
$$

Note that on this KZ spectrum, the particle flux vanishes, i.e., $Q(\omega)=0$ (cf. Eq. (2.26)). 
Similarly to the energy flux, from Eq. (2.19) together with Eq. (2.21), one finds

$$
Q(\omega)=-\frac{2 g^{4} A^{3}}{(4 \pi)^{5}} \frac{\omega^{3 x+4}}{3 x+4} f(x),
$$

which for $x \rightarrow-4 / 3$ yields a constant flux with

$$
\begin{aligned}
\lim _{x \rightarrow-4 / 3} \frac{f(x)}{3 x+4}=\quad & \int_{0}^{1} \mathrm{~d} z_{2} \int_{1-z_{2}}^{1} \mathrm{~d} z_{3} z_{1}^{-1 / 3} z_{2}^{-4 / 3} z_{3}^{-4 / 3} \\
& \times \ln \left(\frac{z_{1}}{z_{2} z_{3}}\right)\left(1+z_{1}^{4 / 3}-z_{2}^{4 / 3}-z_{3}^{4 / 3}\right) \simeq-0.9
\end{aligned}
$$

In agreement with the heuristic argument given in the introduction for the direction of the fluxes, we see that the $\mathrm{KZ}$ spectrum $x=-4 / 3$ is associated with a positive particle flux, $Q(\omega) \equiv Q_{f}>0$, namely, an inverse particle cascade. The corresponding $\mathrm{KZ}$ spectrum then reads

$$
n(\omega)=C_{\mathrm{KZ}} Q_{f}^{1 / 3} g^{-4 / 3} \omega^{-4 / 3}, \quad \text { where } \quad C_{\mathrm{KZ}}=55.8 .
$$

In this case, $P(\omega)=0$.

The convergence of the collision integral on the $\mathrm{KZ}$ spectra is a reflection of the local nature of the interactions in $\phi^{4}$ theory, namely, of the fact that the spectrum is determined by interactions of particles of comparable momenta. For wide enough inertial range, the locations of the source and sinks are then irrelevant and can be sent to 0 and infinity, respectively, for the energy cascade and conversely for the particle cascade.

In the next sections, we shall discuss the kinetic equations that describe a gas of gluons. We shall in particular see that interactions are strongly nonlocal and as a result the steady state spectra differ from the KZ spectra.

\section{Steady state spectra in YM theory: elastic processes}

Let us turn now to classical Yang-Mills (YM) theory. In this section, we shall first discuss in detail elastic processes alone.

At small coupling, $g \ll 1$, the collision integral can be expressed as a powers series in $g^{2}$. The leading order corresponds to elastic 2 to 2 gluon scattering process, whose (squared) matrix element reads

$$
\left|M_{23 \rightarrow 1 k}\right|^{2} \equiv 8 g^{4} N_{c}^{2}\left(3-\frac{t u}{s^{2}}-\frac{u s}{t^{2}}-\frac{s t}{u^{2}}\right) \text {. }
$$


where

$$
s=\left(k+k_{1}\right)^{2}, \quad u=\left(k-k_{3}\right)^{2}, \quad t=\left(k-k_{2}\right)^{2},
$$

are the Mandelstam variables and $k_{i} \equiv\left(\omega_{i}, \boldsymbol{k}_{i}\right)$ are 4 -vectors. Similarly to $\phi^{4}$ theory, the degree of homogeneity of the matrix element (3.1) is zero. Therefore, the latter is invariant under a uniform rescaling of all momenta. Hence, one could expect the power spectra (2.18) derived in the previous section to remain valid for elastic processes in Yang-Mills theories. However, as we shall shortly see, this is not the case since the dominant interactions are nonlocal in momentum space.

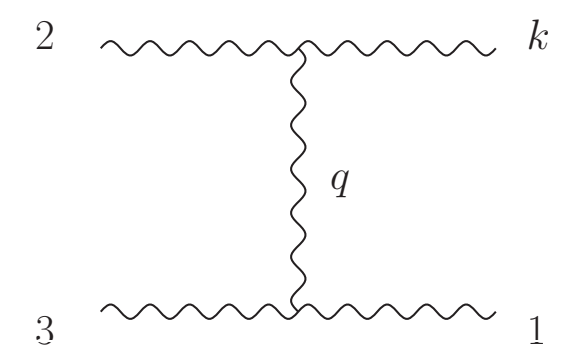

Figure 3: Diagrammatic representation of the dominant contribution to 2 to 2 gluon scattering in the small angle approximation where the momentum transfer $q \ll k_{i}(i=1,2,3)$.

In order to derive the actual turbulent spectrum, we first note that the matrix element (3.1) possesses an infrared singularity in the $t$ and $u$ channels inherent to Coulomb scattering. This implies that a typical scattering is characterized by a small momentum exchange, resulting in a small angle scattering as depicted in Figure 3. Keeping only the singular part and using the symmetry of the kinetic equation under the exchange $u \leftrightarrow t$, Eq. (3.1) yields

$$
\left|M_{23 \rightarrow 1 k}\right|^{2} \simeq 16 N_{c}^{2} g^{4} \frac{s^{2}}{t^{2}}
$$

This divergence should in fact be cutoff at the Debye mass, i.e., $t \rightarrow t+m_{D}^{2}$. Therefore, to apply the small angle approximation, we assume (and will verify it a posteriori) that the screening mass lies beyond the inertial range where 
wave turbulence develop, that is, $k \gg m_{D}$. We can thus perform a gradient expansion around the Coulomb singularity. This power-like divergence present in the gain and loss terms cancels out yielding a milder logarithmic divergence (cutoff at the Debye mass). The details of the calculation is presented in Appendix C. As a result, the 2 to 2 kinetic equation reduces to a Fokker-Planck (FP) equation (also known as the Landau equation) [31, 32]

$$
I^{2 \rightarrow 2}[n] \sim I_{\mathrm{el}}[n] \equiv 4 \pi^{3} \bar{\alpha}^{2} \lambda \boldsymbol{\nabla} \cdot\left[I_{a} \boldsymbol{\nabla} n(k)+\frac{\boldsymbol{k}}{|\boldsymbol{k}|} I_{b} n^{2}(k)\right],
$$

where $\lambda \simeq \ln k / m_{D}$ is the Coulomb logarithm, which we assume to be a constant in what follows, $\bar{\alpha} \equiv g^{2} N_{c} / 4 \pi^{2}$, and

$$
I_{a} \equiv \int \frac{\mathrm{d}^{3} \boldsymbol{p}}{(2 \pi)^{3}} n^{2}(\boldsymbol{p}) \quad \text { and } \quad I_{b} \equiv 2 \int \frac{\mathrm{d}^{3} \boldsymbol{p}}{(2 \pi)^{3}} \frac{n(\boldsymbol{p})}{|\boldsymbol{p}|} .
$$

The first term in Eq. (3.4) describes a process of diffusion in momentum space $k$ and the second term the drag.

Recalling that we focus on isotropic media, it follows that Eq. (3.4) reduces to a one dimensional continuity equation,

$$
I_{\mathrm{el}}[n] \equiv \frac{2 \pi^{2}}{\omega^{2}} \frac{\partial}{\partial \omega} Q(\omega)
$$

where

$$
Q(\omega) \equiv \frac{1}{4} \hat{q} \omega^{2}\left(\frac{\partial}{\partial \omega} n(\omega)+\frac{n^{2}(\omega)}{T_{*}}\right)
$$

stands for the particle flux. Here, we have introduced the diffusion coefficient

$$
\hat{q} \equiv 16 \pi^{3} \bar{\alpha}^{2} \lambda I_{a}
$$

also known as the jet-quenching parameter [33], and the effective temperature

$$
T_{*}=\frac{I_{a}}{I_{b}}
$$

that equals the temperature at thermal equilibrium.

Particle number conservation is straightforward owing to the fact that $\omega^{2} I_{\mathrm{el}}[n]$ is a total derivative. Hence, when the flux vanishes at 0 and $\infty$, we have

$$
\dot{N}=\int \frac{\mathrm{d}^{3} \boldsymbol{k}}{(2 \pi)^{3}} I_{\mathrm{el}}[n]=Q(\infty)-Q(0)=0
$$


Energy conservation relies, on the other hand, on the specific form of the integrals $I_{a}$ and $I_{b}$ that depend globally on the occupation number. We find

$$
\begin{aligned}
\dot{E} & =\int \frac{\mathrm{d}^{3} \boldsymbol{k}}{(2 \pi)^{3}} \omega I_{\mathrm{el}}[n]=\int_{0}^{\infty} \mathrm{d} \omega \omega \frac{\partial}{\partial \omega} Q(\omega)=-\int_{0}^{\infty} \mathrm{d} \omega Q(\omega) \\
& =-\frac{1}{4} \hat{q} \int_{0}^{\infty} \mathrm{d} \omega \omega^{2}\left(\frac{\partial}{\partial \omega} n(\omega)+\frac{n^{2}(\omega)}{T_{*}}\right) \\
& =\frac{1}{4 I_{a}} \hat{q}\left[2 I_{a} \int_{0}^{\infty} \mathrm{d} \omega \omega n(\omega)-I_{b} \int_{0}^{\infty} \mathrm{d} \omega \omega^{2} n^{2}(\omega)\right]
\end{aligned}
$$

where we have repeatedly integrated by part. In the last line, one recognizes the integrals $I_{b}$ and $I_{a}$ defined in Eq. (3.5), in the first and second terms, respectively, that is,

$$
2 \int_{0}^{\infty} \mathrm{d} \omega \omega n(\omega)=2 \pi^{2} I_{b} \quad \text { and } \quad \int_{0}^{\infty} \mathrm{d} \omega \omega^{2} n^{2}(\omega)=2 \pi^{2} I_{a} .
$$

Therefore, we have $\dot{E}=0$.

Eq. (3.6) was studied in the context of Bose Einstein condensation in an overpopulated system of gluons [32]. To accommodate the excess of gluons that do not fit the Bose Einstein distribution at equilibrium, a finite flux of particles builds up a $\omega=0$ to fill the condensate. In the next section, we shall see that an analogous phenomenon occurs in the presence of a steady source.

In the classical regime, for a closed system, the particle flux vanishes identically on the thermal fixed-point (2.9) with $T=T_{*}$. However, an initial distribution with compact support in $\omega$ space never relaxes to thermal equilibrium with a finite value of the temperature. Instead, it evolves in a self-similar way characterized by a spreading of the distribution toward higher frequencies and asymptotically we must have $T_{*}=0$. This is a general feature of decaying wave systems. This can be seen by solving for the temperature the implicit equation Eq. (3.9) where the occupation number $n$ is given by the thermal distribution Eq. (2.9). We find $T_{*}=T=0$.

Of course, when $n \sim 1$ the classical approximation ceases to be valid and the complete quantum statistic must be taken into account. The BoseEinstein distribution is then the true equilibrium distribution characterized by a finite temperature. 


\subsection{Stationary state solutions}

Recall that we are interested in the response of the system when energy is injected at the forcing scale $\omega_{f}$, at a constant rate $P_{f}=Q_{f} \omega_{f}$. If the interactions were local in momentum space one should expect the KZ spectra characterized by the exponents (2.18), to be realized in the inertial range. Obviously, those KZ spectra are not fixed points of Eq. (3.6). Moreover, the integrals $I_{a}$ and $I_{b}$ diverge for any power spectrum and in particular on the KZ spectra $\omega^{-5 / 3}$ and $\omega^{-4 / 3}$. Hence, these integrals strongly depends on boundary conditions, that is, on either the forcing or the dissipation scales. This demonstrates nonlocality of interactions in momentum space for the 2 to 2 matrix element (3.1). Note that the small angle approximation has nothing

to do with the loss of the KZ fixed-points. As a counter example, Kats et al have shown that the stationary solutions of the non-relativistic Landau equation in the low occupancy limit are indeed given by the corresponding local KZ spectra $\omega^{-5 / 4}$ and $\omega^{-3 / 4}[19]$.

In order to investigate the steady state spectra associated with a finite flux we add a spectrally narrow source term to the collision term (3.6),

$$
\frac{\partial n(\omega)}{\partial t}=I_{\mathrm{el}}[n]+S(\omega)
$$

where the source term

$$
S(\omega)=\frac{2 \pi^{2} Q_{f}}{\omega^{2}} \delta\left(\omega-\omega_{f}\right)
$$

is normalized to yield the particle flux as follows

$$
Q_{f}=\int \frac{\mathrm{d}^{3} \boldsymbol{k}}{(2 \pi)^{3}} S(\omega)
$$

In the presence of a source and sinks we expect the system to reach a stationary state after a transient regime. We find that the stationary solutions of Eq. (3.13) obey a Ricatti type differential equation

$$
Q=\frac{1}{8 \pi^{2}} \hat{q} \omega^{2}\left(\frac{\partial}{\partial \omega} n(\omega)+\frac{n^{2}(\omega)}{T}\right)+Q_{f} \Theta\left(\omega-\omega_{f}\right)=\text { const }
$$

which necessarily implies a constant flux of particles. Hence, there is no solution explicitly related to energy conservation which is accounted for globally 
through a implicit dependence of the integrals $I_{a}$ and $I_{b}$ on $n$. Curiously, as we shall shortly show, this fact implies the vanishing of the spectrum above the forcing scale where the energy cascade forms.

The exact solution of Eq. (3.16) is derived in Appendix D (cf. Eqs. (D.15) and (D.16)). Here, we only focus on the homogeneous (power like) solution, that can be inferred from the exact solution far from the source and sinks. For $\omega_{-} \ll \omega<\omega_{f}$, below the forcing scale, we obtain

$$
n(\omega)=\frac{A}{\omega}, \quad \text { with } \quad A=\frac{T_{*}}{2}\left(1+\sqrt{1+\frac{32 \pi^{2} Q}{\hat{q} T_{*}}}\right),
$$

and for $\omega_{f}<\omega \ll \omega_{+}$, above the forcing scale,

$$
n(\omega)=\frac{B}{\omega}, \quad \text { with } \quad B=\frac{T_{*}}{2}\left(1-\sqrt{1+\frac{32 \pi^{2}\left(Q-Q_{f}\right)}{\hat{q} T_{*}}}\right) .
$$

Note that $A>B$ and thus, the solution is discontinuous at the forcing scale, $\omega_{f}$. In the absence of an extra boundary condition to fixe the value of $Q$, we conclude that it should depend on initial conditions and the location of the sinks. Moreover, since particle number must flow toward the infrared, we expect $Q \simeq Q_{f}>0$. As a result, the steady state solution for large inertial range simply reads

$$
n(\omega)=\left\{\begin{array}{cc}
\frac{1}{2}\left(1+\sqrt{\left.1+\frac{32 \pi^{2} Q_{f}}{\hat{q} T_{*}}\right)} \frac{T_{*}}{\omega}\right. & \text { for } \omega<\omega_{f} . \\
0 & \text { for } \omega>\omega_{f} .
\end{array}\right.
$$

Of course, energy is conserved and carried by the modes at the ultraviolet boundary, $\omega_{+}$. The vanishing of the occupation number to the right of $\omega_{f}$ reflects the fact that it must depend on the location of the sink $\omega_{+}$where energy is dissipated. Note also that $T_{*}$ and $\hat{q}$ depend implicitly on $n$. We shall discuss these points, and in particular the approach to the steady spectrum (3.19), in more detail in what follows.

\subsection{Approach to the steady state}

Here, we shall perform numerical simulations (see Appendix $\mathrm{H}$ for details) of the Fokker-Planck equation (3.13) to study the approach toward the steady 
state (3.19). The results will be plotted as a function of the rescaled time variable

$$
\tau=4 \pi^{3} \bar{\alpha}^{2} \lambda t
$$

Furthermore, we set $\omega_{f}=1$ and $Q_{f}=1$ and the source is modeled by a narrow Gaussian

$$
S(\omega)=\frac{2 \pi^{2} Q_{f}}{\sqrt{2 \pi} \sigma \omega_{f}^{2}} \exp \left[\left(\omega-\omega_{f}\right)^{2} / 2 \sigma^{2}\right]
$$

with $\sigma^{2}=0.05$. The sinks are accounted for by removing particles outside the inertial range, that is, letting $n(\omega)=0$, for $\omega>\omega_{+}=500$ and $\omega<\omega_{-}=0.02$. Because the sources are not exactly vanishing at $\omega=0$, Eq. (3.21) yields the numerical values $Q_{f} \simeq 0.9$ and $P_{f} \simeq 1$ (see inset in Figure 4).

The late time evolution of the full spectrum is shown in Figure 4 for $\tau=$ 102, 1076 and 100057. We have checked that the spectrum to the left of the forcing scale $\left(\omega<\omega_{f}=1\right)$ agrees with Eq. (3.19) with $A \simeq 1.17$ where $\hat{q} \simeq 4.60, T_{*} \simeq 0.09$ and $Q=Q_{f} \simeq 0.9$ are computed dynamically, evaluated at $\tau=256504$ using the numerical solution for $n(\omega)$ in Eqs. (3.5), (3.8) and (3.9). To the right of the forcing scale, we see that the distribution decreases with time and thus, is expected to vanish asymptotically matching the stationary solution (3.19).

Moreover, we confirm that $Q \simeq Q_{f}-0.9$ and $P \simeq 0$, to the left of the forcing scale as shown in Figure 4 (inset), which corresponds to the inverse particle cascade. Conversely, to right of the forcing scale, we should have $Q \simeq 0$ and $P \simeq P_{f}$. To the right of the forcing scale, $Q \simeq 0$ and $P \simeq P_{f} \approx 1$, reflecting the energy transfer to the ultraviolet. Also, we have also analyzed the early times dynamics. In the infrared sector, i.e. $\omega<\omega_{f}$, the power spectrum $\omega^{-1}$ can contain a finite number of particles, thus, it is of finite capacity. A finite flux of particle fills the entire spectrum all the way down to $\omega=0$ (in the absence of damping) in a finite amount of time $t_{c}$, which corresponds to the time it takes for a finite flux of particle to develop at the origin. To see this, we have plotted the evolution of spectrum before the onset of condensation in Figure 5.

This phenomenon is akin to Bose-Einstein condensation and has been discussed in Ref. [23, 32] (see also [30] for a detailed discussion of BEC in the framework of the Boltzmann-Nordheim equation). In this case the steady state is not sensitive to position of the infrared sink $\omega_{-} \ll \omega_{f}$. 


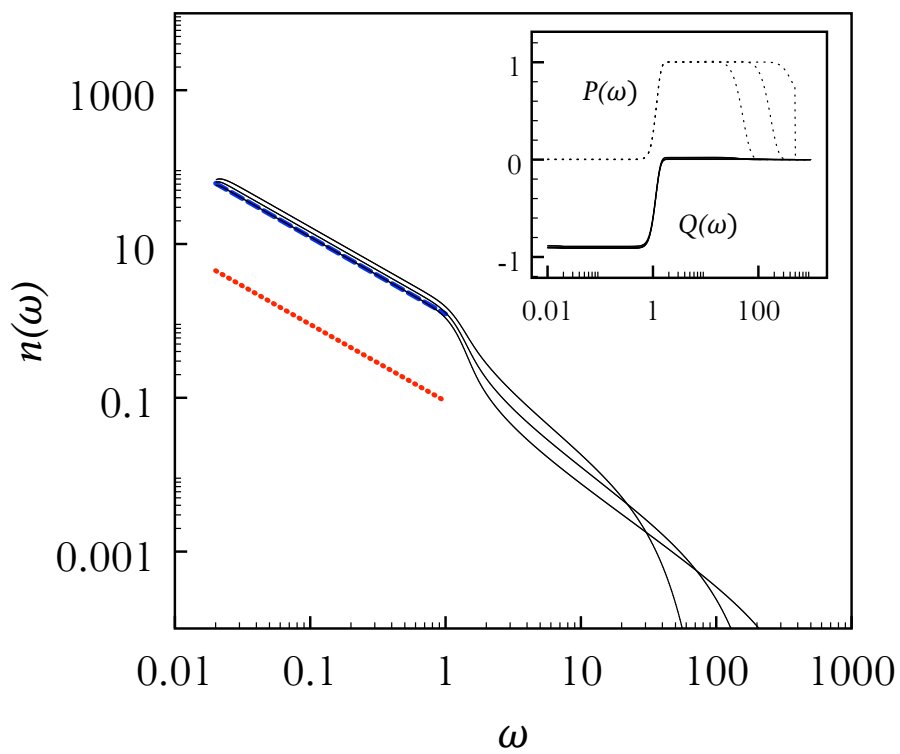

Figure 4: Numerical solution of the Fokker-Planck equation (3.13) in the presence of a source at $\omega_{f}=1$, pumping particles at a rate $Q_{f} \simeq 0.9$. The occupation number at late times is depicted by full (black) curves corresponding to $\tau=1018,16286$ and 256504, from top to bottom. The dashed (blue) curve is the asymptotic spectrum Eq. (3.19). The dotted (red) curve corresponds to the thermal spectrum $T_{*} / \omega$ with $T_{*} \simeq 0.09$ (at $\tau=256504$ ) and shown as a reference. In the inset, the corresponding particle flux (full curves) and energy flux (dotted curves) are plotted.

Because the occupation number is expected to drop to the right of the forcing scale at late times, it is reasonable to assume and verify it afterwards that the section $\omega>\omega_{f}$ does not contribute asymptotically to the integrals $I_{a}$ and $I_{b}$. Under this assumption we have

$$
I_{a} \sim \int_{0}^{\omega_{f}} \mathrm{~d} \omega \omega^{2} n^{2} \sim A^{2} \omega_{f} \quad \text { and } \quad I_{b} \sim \int_{0}^{\omega_{f}} \mathrm{~d} \omega \omega n \sim A \omega_{f}
$$

where we have used Eq. (3.17) in Eq. (3.12). Therefore, we have, parametrically, $A \sim T_{*}$. However, because there is a finite flux of particles, we must 


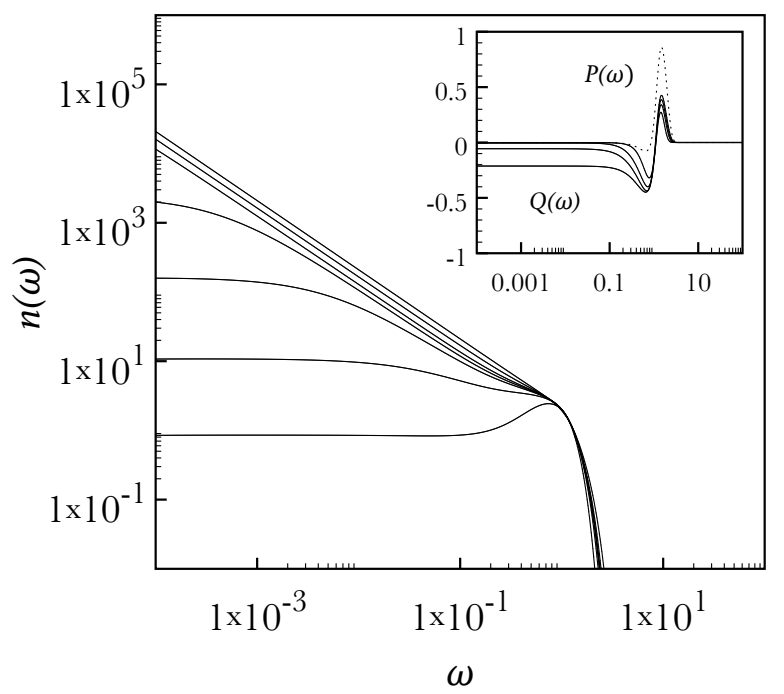

Figure 5: Time evolution of the occupation number before the onset of condensation. From bottom to top $\tau=1.00,1.20,1.26,1.28,1.30,1.35$ and 1.50. In the inset, the time evolution of the particle flux (full lines from top to bottom $\tau=1.00,1.20,1.28$ and 1.35) and energy flux (dashed line for $\tau=1.35$ ) (see also Figure 4 for late time behavior), is shown. The asymptotic $\omega^{-1}$ spectrum forms after the onset of condensation, at $\tau \gtrsim 1.3$, that is when the particle flux $Q \neq 0$ at $\omega=0$.

have $A>T$. Putting all the pieces together, Eqs. (3.17) and (3.22) yield

$$
n(\omega)=\frac{A}{\omega} \quad \text { with } \quad A \sim \frac{Q_{f}^{1 / 3}}{\bar{\alpha}^{2 / 3} \omega_{f}^{1 / 3}} .
$$

This spectrum corresponds to the nonlocal turbulent spectrum associated with the inverse particle cascade. The nonlocality is reflected in the explicit dependence of the occupation number on the forcing scale.

To right of the forcing scale the time evolution of the spectrum is controlled by a diffusion process toward to ultraviolet. As shown in Figure 4, a power spectrum forms in the wake of a wave front that increases with time as

$$
\omega_{*}(t) \sim \sqrt{\hat{q} t}
$$


with

$$
\hat{q} \sim \bar{\alpha}^{2} A^{2} \omega_{f} \sim \bar{\alpha}^{2 / 3} Q_{f}^{2 / 3} \omega_{f}^{1 / 3}
$$

It remains to analyze the evolution of the constant $B$ and verify that $B=0$ is indeed an attractor. Note that because the slope of the spectrum is larger that -4 , the total energy contained in the spectrum is dominated by the ultraviolet cutoff $\omega_{*}$. Hence, the total energy injected in the system at the time $t$ reads

$$
E \sim P_{f} t \sim \int_{0}^{\omega_{*}(t)} \mathrm{d} \omega \omega^{3} n^{2} \simeq \omega_{*}^{3}(t) B .
$$

Since $\omega_{*} \sim t^{1 / 2}$, it follows that the spectrum to the right of the forcing scale decreases with $t$ as $B \sim t^{-1 / 2}$. The wave front reaches the sink, $\omega_{*} \sim \omega_{+}$, at the time $t_{+} \sim \omega_{+}^{2} / \hat{q}$. So, letting $t=t_{+}$in Eq. (3.26) and solving for $B$, we obtain

$$
n(\omega)=\frac{B}{\omega} \quad \text { with } \quad B \sim \frac{Q_{f}^{1 / 3} \omega_{f}^{2 / 3}}{\bar{\alpha}^{2 / 3} \omega_{+}} .
$$

Therefore, the turbulent spectrum is suppressed to the right of the forcing scale in the limit $\omega_{+} \rightarrow \infty$. As a consistency check, it can be verified that the contribution of the section $\omega>\omega_{f}$ to the integrals $I_{a}$ and $I_{a}$ is suppressed in for the former and independent of $\omega_{+}$for the latter, as assumed in Eq. (3.22).

\section{Steady state spectrum in Yang-Mills: inelastic processes}

Higher order corrections to the collision integral involve inelastic processes in general, and hence do not conserve particle number. Naively, one expects these correction to be suppressed owing to the fact that an extra gluon radiation (absorption) costs a factor $g^{2} n \ll 1$. However, as alluded to in the introduction, inelastic processes are not suppressed by higher powers of the coupling constant and must be taken into account for a complete treatment. In effect, the 2 to 3 gluons matrix element possesses a collinear divergences associated with Coulomb scattering and gluon branching, which is be regulated by the Debye mass and the gluon thermal, respectively. This effect is non-perturbative, as it results from the resummation of hard loops in the gluon propagator. Contrary to the elastic scattering this power divergence 
does not reduce to a logarithmic one thanks to cancellations between the gain and loss terms. The angular (or transverse momentum) integration generates a factor $1 / m^{2}$ in the collision integral and because $m^{2} \sim g^{2} n$ (cf. Eq. (1.4)), inelastic 2 to 3 processes turn out to be parametrically as important as elastic 2 to 2 processes (cf. Appendix E).

Hence, in 2 to 3 processes, depicted in Figure 6 (a), one of the out going gluons tends to be collinear to one of the incoming ones, for instance gluons 2 and 4 . Conservation of the total 3-momentum implies that the three remaining gluons, 1,3 and $k$, are also nearly collinear. So effectively, keeping only the singular part this process can be regarded as a quasi-collinear 1 to 2 splitting (of gluon 1 into 3 and $k$ ) induced by the small angle scatterings off gluons (2) whose momentum is integrated out (a diagram representing this process is shown in Figure $6(\mathrm{~b}))$.

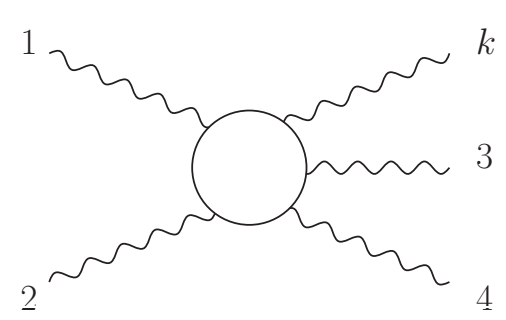

(a)

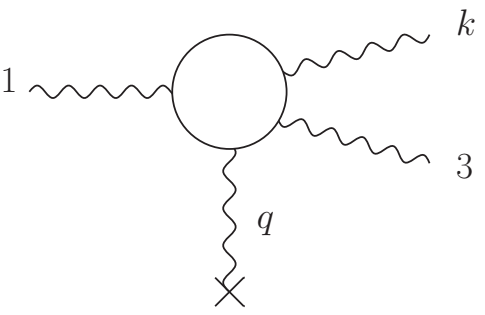

(b)

Figure 6: (a): Diagrammatic representation of the 2 to 3 matrix element. (b): The leading contribution to the 2 to 3 process corresponding to small momentum exchange $q \ll \omega_{i}(i=1,2,3,4, k)$. In this limit, gluons 1,3 and $k$ are quasi-collinear. The cross at the end of the vertical gluon line illustrates the fact that the collinear gluons 2 and 4 are integrated over so that their respective momenta do not affect the (upper) branching process.

This quasi-collinear splitting was largely discussed in the context of inmedium radiative energy loss (see Refs. $[34,35]$ for a recent review and references therein) developed in the mid 90's then applied to the problem of thermalization of the quark-gluon plasma where the effective theory used in this work was proposed $[13,21]$.

A standard approach that accounts for the small angle scattering by construction consists in computing the 1 to 2 splitting in the presence of a stochastic background field, $A_{0}^{\mu}(q)$ generated by the hard gluons, with $q \sim m$, 
whose two-point correlation function is given by

$$
\left\langle A_{0}^{\mu, a}(Q) A_{0}^{* \nu, b}\left(Q^{\prime}\right)\right\rangle \equiv(2 \pi)^{4} \delta^{(4)}\left(Q-Q^{\prime}\right) \frac{\delta^{a b}}{g^{2} N_{c}} v^{\mu} v^{\nu} \mathcal{C}\left(q_{\perp}\right),
$$

where for an isotropic plasma

$$
\mathcal{C}\left(q_{\perp}\right) \equiv \frac{g^{2} N_{c} T_{*} m_{D}^{2}}{q_{\perp}^{2}\left(q_{\perp}^{2}+m_{D}^{2}\right)},
$$

in the Hard-Loop approximation $[36,21]$. Here, the momentum transfer $q_{\perp}$ is transverse to the momenta of the collinear gluons in the 1 to 2 process. The $v^{\mu} v^{\nu}$ factor in the r.h.s. of Eq. (4.1), where $v^{\mu} \equiv k^{\mu} /|\boldsymbol{k}|$ is the gluon velocity, stems from the eikonal coupling (small angle scattering) of high energy gluons, with the soft collective modes of the background field $q \sim$ $m \ll k$.

Integrating the function $\mathcal{C}$ over $q_{\perp}$ yields the inverse elastic mean-freepath,

$$
\ell_{\mathrm{mfp}}^{-1} \sim \int_{\boldsymbol{q}_{\perp}} \mathcal{C}\left(q_{\perp}\right) \sim g^{2} T_{*}
$$

where we have used the shorthand notation $\int_{\boldsymbol{q}_{\perp}} \equiv \int \mathrm{d}^{2} \boldsymbol{q} /(2 \pi)^{2}$. The effective temperature $T_{*}$ and the Debye mass $m_{D}$ are given by Eq. (3.9) and Eq. (1.4), respectively.

From the uncertainty principle, the time it takes for a gluon $\omega$ to split into two gluons sharing fractions $z$ and $1-z$ of its energy reads

$$
t \sim \frac{z(1-z) \omega}{k_{\perp}^{2}}
$$

where $k_{\perp}$ is the transverse momentum generated in the branching process. When the branching process is triggered by many coherent scatterings the transverse momentum squared accumulated is typically $k_{\perp}^{2} \sim \hat{q} t$, which together with Eq. (4.4) yields the coherence time

$$
t=t_{\mathrm{coh}} \sim \sqrt{\frac{z(1-z) \omega}{\hat{q}}} .
$$

There exist two regimes that are characterized by the largest of either $\ell_{\mathrm{mfp}}$ or $t_{\mathrm{coh}}$, and which we summarize in the following: 
1. The Bethe-Heitler $(\mathrm{BH})$ regime: when $t_{\text {coh }} \ll \ell_{\text {mfp }}$, that is, $\omega \ll$ $\omega_{\mathrm{BH}} \sim \hat{q} \ell_{\mathrm{mfp}}^{2}$, the collinear branching involves a single scattering. Using Eqs. (3.8), (3.9) and (4.3), we find that $\omega_{\mathrm{BH}}$ depends only on the integrals $I_{a}$ and $I_{b}$ (cf. 3.5),

$$
\omega_{\mathrm{BH}} \sim \frac{I_{b}^{2}}{I_{a}} .
$$

In this regime, the matrix element squared can be approximated by the collinear limit of the 2 to 3 matrix element with proper treatment of screening effects (cf. Appendix F). The rate for the branching of a gluon of frequency $\omega_{1}=\omega / z$ into two offsprings with frequencies $\omega$ and $\omega_{1}=(1-z) \omega / z$ as shown in Figure $6(\mathrm{~b})$, scales as $\mathcal{K}_{\mathrm{BH}} \sim \bar{\alpha} / \ell_{\mathrm{mfp}}$ (see Eq. (4.11)).

2. The Landau-Pomeranchuk-Migdal (LPM) regime: when $t_{\text {coh }} \gg \ell_{\mathrm{mfp}}$ or $\omega \gg \omega_{\mathrm{BH}}$, multiple scatterings off the medium constituents occur during the branching process depleting the inelastic rate compared to the incoherent $\mathrm{BH}$ process (cf. Figure 7). In this case, the matrix element includes singular contributions from higher order processes of the form $N+1$ to $N+2$. In this coherent limit, the branching rate scales as $\mathcal{K}_{\mathrm{LPM}} \sim \bar{\alpha} / t_{\text {coh. }}$. In turns out that in this case the branching rate is only logarithmically sensitive to the screening mass.

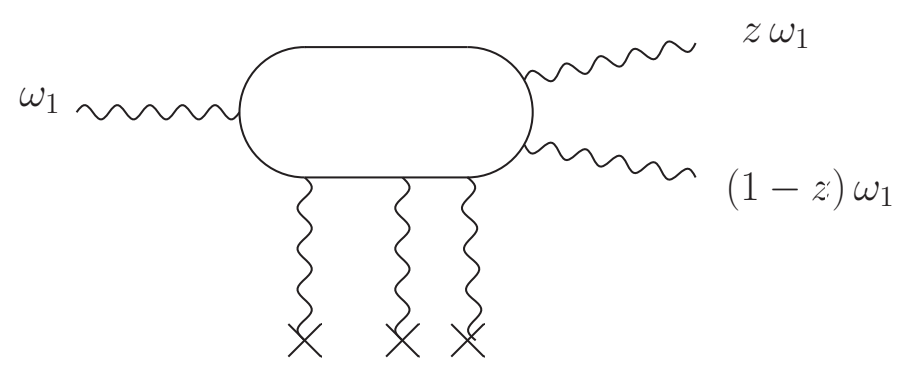

Figure 7: Similar to Figure 6(b) in the regime of multiple small momentum exchanges, that is the LPM regime. Here only three scatterings are depicted.

The branching rate, first derived by Baier et al [44] and Zakharov [45], 
can be easily recovered from the literature ${ }^{5}$. It reads, ${ }^{6}$

$$
\mathcal{K}(z) \equiv \frac{2 \alpha_{s}}{z^{2}(1-z)^{2} \omega_{1}^{2}} P(z) \operatorname{Re} \int_{0}^{\infty} \mathrm{d} \tau \int_{\boldsymbol{p}_{\perp}, \boldsymbol{q}_{\perp}}\left(\boldsymbol{p}_{\perp} \cdot \boldsymbol{q}_{\perp}\right) S\left(\boldsymbol{p}_{\perp}, \boldsymbol{q}_{\perp}, z, \tau\right),
$$

where,

$$
P(z)=2 N_{c}\left[\frac{1-z}{z}+\frac{z}{1-z}+z(1-z)\right],
$$

is the Altarelli-Parisi gluon-gluon splitting function. The Green's function $S$ obeys the two dimensional Schrödinger equation

$$
\begin{aligned}
& {\left[i \frac{\partial}{\partial \tau}-\frac{\boldsymbol{p}_{\perp}^{2}+m_{z}^{2}}{2 z(1-z) \omega_{1}}\right] S\left(\boldsymbol{p}_{\perp}, \boldsymbol{q}_{\perp}, z, \tau\right)=} \\
& +\frac{i}{2} \int_{\boldsymbol{l}_{\perp}}\left[\tilde{\mathcal{C}}\left(\boldsymbol{l}_{\perp} /(1-z)\right)+\tilde{\mathcal{C}}\left(\boldsymbol{l}_{\perp} / z\right)+\tilde{\mathcal{C}}\left(\boldsymbol{l}_{\perp}\right)\right] S\left(\boldsymbol{p}_{\perp}-\boldsymbol{l}_{\perp}, \boldsymbol{q}_{\perp}, z, \tau\right),
\end{aligned}
$$

with the initial condition $S\left(\boldsymbol{p}_{\perp}, \boldsymbol{q}_{\perp}, z, \tau\right)=(2 \pi)^{2} \delta^{(2)}\left(\boldsymbol{p}_{\perp}-\boldsymbol{q}_{\perp}\right)$. Here, $m_{z}^{2}=$ $\left(1-z+z^{2}\right) m^{2}$ where $m^{2}$ is the gluon thermal mass squared (cf. Eq. (1.4)) and

$$
\tilde{\mathcal{C}}\left(\boldsymbol{l}_{\perp}\right) \equiv \mathcal{C}\left(\boldsymbol{l}_{\perp}\right)-(2 \pi)^{2} \delta^{(2)}\left(\boldsymbol{l}_{\perp}\right) \int_{\boldsymbol{l}_{\perp}^{\prime}} \mathcal{C}\left(\boldsymbol{l}_{\perp}^{\prime}\right)
$$

In Eq. (4.7), $\tau$ stands for the time between the branchings in the amplitude and the complex conjugate amplitude where only the transverse momentum of gluon $z$ is measured and $q_{\perp}$ and $p_{\perp}$ are respectively its initial and final transverse momenta relative to the parent gluon. The Green's function (4.9) describes the in-medium propagation, characterized by the differential scattering rate $\mathcal{C}$ of the system made of the two offspring gluons (carrying the

\footnotetext{
${ }^{5}$ Different notations were adopted in the literature making it difficult to connect directly to the present work which is based on the derivation presented in [37]. The facilitate the task we refer the reader to Ref. [38] where a useful dictionary relating the various approaches is provided.

${ }^{6}$ The branching rate $\mathcal{K}(z) \equiv \mathcal{K}\left(z, \omega_{1}\right)$ is an implicit function of the energy of the parent gluon $\omega_{1}$.
} 
energy fractions $z$ and $1-z$ of the parent gluon) in the amplitude and the parent gluon in the complex conjugate amplitude (assuming that the branching in the amplitude occurs first).

Analytic solutions of Eq. (4.9) can only be obtained in the limiting cases aforementioned. This is worked out in Appendix F. In the Bethe-Heitler regime the branching kernel reads

$$
\mathcal{K}(z) \simeq \frac{\pi \bar{\alpha}^{2} C T_{*}}{N_{c}} P(z),
$$

where $C \simeq 1.83$ and in the $\mathrm{LPM}$ regime it reads

$$
\mathcal{K}(z)=\bar{\alpha} P(z) \sqrt{\frac{\hat{q}\left(1-z+z^{2}\right)}{z(1-z) \omega_{1}}} .
$$

Let us turn now to the effective kinetic equation corresponding to the quasi-collinear 1 to 2 process. In general, the 3-wave collision integral takes the form

$$
I_{\text {inel }}[n]=\frac{1}{\omega^{2}} \int\left(R_{12 \omega}+R_{1 \omega 2}-R_{\omega 12}\right) \mathrm{d} \omega_{1} \mathrm{~d} \omega_{2}
$$

where

$$
R_{12 \omega} \equiv V_{12 \omega} F_{12 \omega} \delta\left(\omega_{1}-\omega_{2}-\omega\right)
$$

with

$$
\begin{aligned}
F_{12 \omega} & =n_{1}\left(n_{2}+1\right)\left(n_{\omega}+1\right)-\left(n_{1}+1\right) n_{2} n_{\omega} \\
& \simeq n_{1} n_{2}+\left(n_{1}-n_{2}\right) n_{\omega},
\end{aligned}
$$

in the overoccupied case, i.e., $n \gg 1 .^{7}$. The prefactor $1 / \omega^{2}$ ensures energy conservation:

$$
\frac{\partial E}{\partial t} \equiv \frac{1}{2 \pi^{2}} \int_{0}^{\infty} \mathrm{d} \omega \omega^{3} I_{\text {inel }}[n]=0 .
$$

\footnotetext{
${ }^{7}$ The underoccupied case was discussed in Refs. [39] and [40], in the context of parton cascade evolution in a dense medium. There, it is shown that an inverse energy cascade forms, that is characterized by (local) democratic branchings with KZ exponent $-7 / 2$ ( or $-1 / 2$ for the distribution $D(\omega) \equiv \omega \mathrm{d} N / \mathrm{d} \omega)$.
} 
The matrix element $V$ relates to the branching rate $\mathcal{K}$ as follows,

$$
V_{12 \omega} \equiv \frac{1}{2} \omega_{1} \mathcal{K}\left(\frac{\omega}{\omega_{1}}\right)
$$

Using the symmetry in the exchange $1 \leftrightarrow 2$, we readily see that the first two terms in the r.h.s of Eq. (4.13) are equal, that is, $R_{12 \omega}=R_{1 \omega 2}$. In the last term, multiplying the integrant by $1 \equiv\left(\omega_{1}+\omega_{2}\right) / \omega$, then replacing the dummy variable $\omega_{2}$ by $\omega_{1}$ in the second term (owing to the aforementioned symmetry), amounts to the following replacement

$$
V_{\omega 12} \rightarrow \frac{2 \omega_{1}}{\omega} V_{\omega 12}
$$

Thereby, Eq. (4.13) can be rewritten as

$$
\begin{aligned}
I_{\text {inel }}[n]= & \frac{2}{\omega^{2}} \int\left[V_{12 \omega} F_{12 \omega} \delta\left(\omega_{1}-\omega_{2}-\omega\right)\right. \\
& \left.-\frac{\omega_{1}}{\omega} V_{\omega 12} F_{\omega 12} \delta\left(\omega-\omega_{1}-\omega_{2}\right)\right] \mathrm{d} \omega_{1} \mathrm{~d} \omega_{2}
\end{aligned}
$$

After integrating over $\omega_{1}$ using the delta functions and defining the kernel:

$$
K\left(\omega, \omega_{2}\right) \equiv \omega_{1} \omega \mathcal{K}\left(\frac{\omega}{\omega_{1}}\right)=2 \omega V_{12 \omega}
$$

one can express Eq. (4.19) as follows (with $q=\omega_{2}$ ),

$$
\begin{aligned}
& I_{\text {inel }}[n]=\frac{1}{\omega^{3}}\left[\int_{0}^{\infty} \mathrm{d} q K(\omega, q) F(\omega+q, \omega)\right. \\
&\left.\quad-\int_{0}^{\omega} \mathrm{d} q K(\omega-q, q) F(\omega, \omega-q)\right],
\end{aligned}
$$

Here, it is understood that

$$
F(\omega+q, \omega) \equiv F_{\omega+q, \omega, q}=n(\omega+q) n(\omega)+[n(\omega+q)-n(\omega)] n(q) .
$$

and similarly for $F(\omega, \omega-q)$. 
A few remarks are in order. Eq. (4.21) contains four contributions. The first term corresponds to rate for producing a gluon $\omega$ from a higher excitation $\omega+q$. It involves a gain term (positive part of Eq. (4.22)) that enhances the occupancy at frequency $\omega$ by branching of a gluon $\omega+q$ into two gluons $\omega$ and $q$, and a loss term that corresponds to the inverse process, that is, gluon the merging of gluon $\omega$ with a gluon $q$ to from a the harder gluon $\omega+q$. The latter contribution is related to the negative part of Eq. (4.22). The second term in Eq. (4.21) accounts for interactions between the $\omega$ bin with lower frequencies, it is related to the first term through the transformation $\omega \rightarrow \omega+q$. A depiction of these four components is shown in Figs. 8 and 9 .

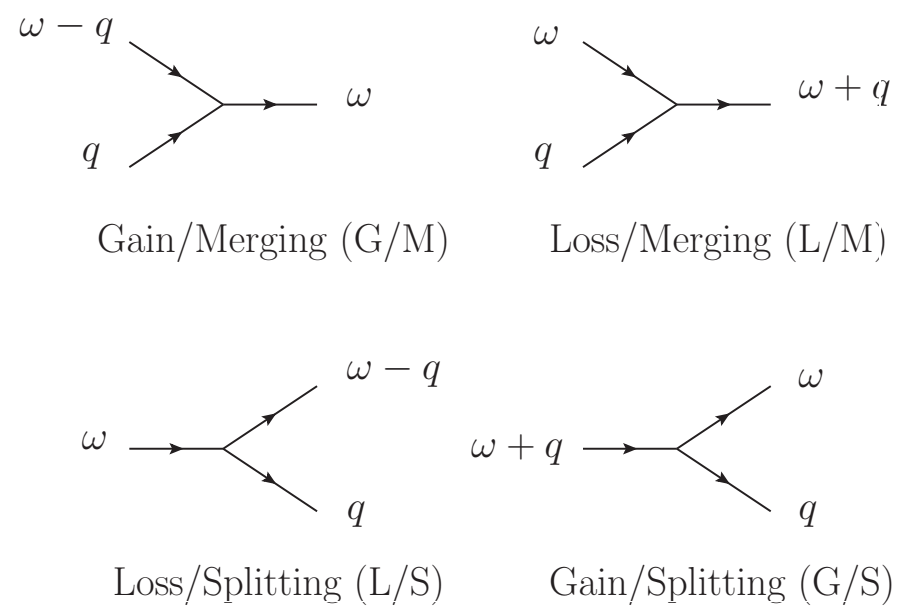

Figure 8: The four contributions to the three wave collision integral.

\subsection{Nonlocality of $K Z$ spectra for the effective 1 to 2 gluon process}

As we have discussed above the kernel $K$ is not scale invariant due to the existence of two regimes, the $\mathrm{BH}$ and LPM regimes that are separated by the frequency $\omega_{\mathrm{BH}}$. Nevertheless, assuming that either $\omega \ll \omega_{\mathrm{BH}}$ or $\omega \gg \omega_{\mathrm{BH}}$, the Kernel becomes a homogeneous function of the frequencies and one can look for $\mathrm{KZ}$ spectra in the respective regimes.

In order to generically study the fixed points of Eq. (4.21) it is useful to parametrize the Kernel such that it includes both cases. In addition, and without loss of generality we shall consider a simplified kernel that captures correctly the limit $z \rightarrow 0$ and $z \rightarrow 1$, by making the replacement $P(z) \rightarrow$ 


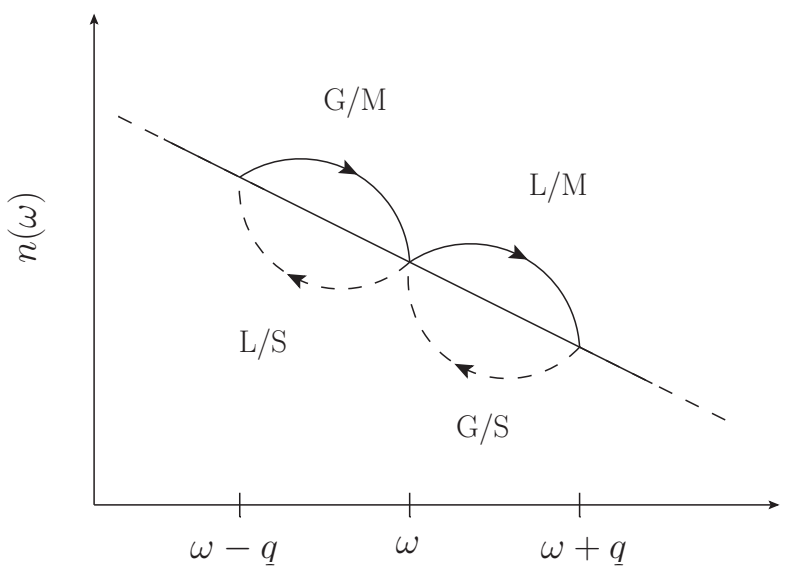

Figure 9: A sketch of the occupation number with an illustration of the four processes (cf. Figure 8) involved in its time evolution: gain and loss by merging and splitting are depicted, respectively, by $\mathrm{G} / \mathrm{M}$ and $\mathrm{L} / \mathrm{M}$ (full arrows), and by G/S, L/S (dashed arrows).

$2 N_{c} / z(1-z)$ and $1-z-z^{2} \rightarrow 1$. Hence, we define

$$
K(\omega, q) \equiv \kappa \frac{(\omega+q)^{3+\beta}}{\omega^{\beta} q^{1+\beta}} .
$$

From Eqs. (4.11) and (4.12), we read off $\beta=0$ and $\kappa \equiv 2 \pi \bar{\alpha}^{2} C T_{*}$ in the BH regime, and $\beta=1 / 2$ and $C=2 N_{c} \bar{\alpha} \sqrt{\hat{q}}$, in the LPM regime.

Because the quasi-collinear 1 to 2 process under consideration conserves energy only, one expects a single turbulent spectrum associated with a finite flux of energy throughout scales from the source $\omega_{f}$ to the sink. To derive the corresponding KZ spectrum, it is useful to return to the $z$ variable, which is equivalent to performing a Zakharov conformal transformation, as in Appendix A, for 3-wave interaction collision integral. Thus, let $z=\omega /(\omega+q)$ in the gain term and $z=(\omega-q) / \omega$ in the loss term. Under this change of variables the collision integral (4.21) becomes

$$
\frac{\kappa}{\omega^{\beta}}\left[\int_{0}^{1} \mathrm{~d} z \frac{1}{z^{4}(1-z)^{1+\beta}} F(\omega, \omega / z)-\int_{0}^{1} \mathrm{~d} z \frac{1}{z^{\beta}(1-z)^{1+\beta}} F(z \omega, \omega)\right] .
$$


Following Zakharov's method, we assume a power spectrum

$$
n(\omega)=A \omega^{x},
$$

and look for $x$ that cause the collision integral to vanish or, equivalently, that generates a constant flux of energy,

$$
P(\omega)=P=\text { const. }
$$

Using Eq. (4.24) in Eq. (2.21), one obtains

$$
P(\omega)=\kappa \int_{0}^{1} \mathrm{~d} z \frac{1}{z^{\beta}(1-z)^{1+\beta}} \int_{\omega}^{\omega / z} d \omega_{0} \omega_{0}^{3-\beta} F\left(z \omega_{0}, \omega_{0}\right),
$$

with

$$
F\left(z \omega_{0}, \omega_{0}\right)=A^{2} \omega_{0}^{2 x} z^{x}(1-z)^{x}\left[(1-z)^{-x}+z^{-x}-1\right] .
$$

Note first that $F$ vanishes identically (and hence the flux $P$ ) at the thermal fixed point $x=-1$. To obtain the non-thermal fixed point, one requires the energy flux to be independent of $\omega$. Therefore, the $\omega_{0}$ integral must yield

$$
\int_{\omega}^{\omega / z} \mathrm{~d} \omega_{0} \omega_{0}^{3-\beta+2 x}=\int_{\omega}^{\omega / z} \frac{\mathrm{d} \omega_{0}}{\omega_{0}}=\ln \frac{1}{z},
$$

which implies

$$
x=\frac{\beta-4}{2} .
$$

This corresponds to the KZ solutions

$$
n(\omega)= \begin{cases}\omega^{-2} & \text { for } \quad \beta=0 \quad(\text { BH regime }) \\ \omega^{-7 / 4} & \text { for } \quad \beta=1 / 2 \quad \text { (LPM regime) },\end{cases}
$$

which are associated with a constant and negative energy flux $P(\omega) \equiv-P_{f}<$ 0 ,

$$
P_{f}=A^{2} \kappa \int_{0}^{1} \mathrm{~d} z \frac{(1-z)^{-x}+z^{-x}-1}{z^{\beta-x}(1-z)^{1+\beta-x}} \ln \frac{1}{z},
$$

that is, with a direct energy cascade. 
The physical relevance of the KZ solution relies on the convergence of the $z$ integral on the KZ spectra. For $x<-1$, we see that this is only the case when $\beta-x-2<0$, i.e., $\beta<0$ and $x<-2$. This condition is therefore not fulfilled by the KZ spectra (4.31). Nevertheless, we observe that the energy flux for the $\mathrm{KZ}$ spectrum $\omega^{-2}$ in the $\mathrm{BH}$ regime diverges double logarithmically, as $P \sim \int_{0} \mathrm{~d} z / z \ln (1 / z)$. As a result, it is marginally nonlocal and thus, can be corrected, to yield a constant flux, by a mild logarithmic dependence on the forcing scale, that is, $n(\omega) \sim \omega^{-2} \ln ^{-1}\left(\omega / \omega_{f}\right)$ [41].

As a proof of principle, we have computed the $\mathrm{KZ}$ constant for the local KZ spectrum $x=-5 / 2$ associated with $\beta=-1$. Integrating Eq. (4.32) and solving for $A$ we find $A \equiv A_{\mathrm{KZ}}=1.1$. In Figure 10, we compare the time evolution of the occupation number, computed by solving the 3 -wave kinetic equation numerically, the analytic KZ spectrum shown by the blue (dashed) line.

Let us now briefly comment on the transition between the $\mathrm{BH}$ and the LPM regimes. The BH frequency (4.6) is invariant under a uniform rescaling of the occupation number, therefore, it should be independent of the energy flux, $P_{f}$. Moreover, when the occupation number is steeper than $\omega^{-2}$, then $\omega_{\mathrm{BH}} \sim \omega_{f}$, parametrically. Hence, for a large inertial range $\omega_{f} \ll \omega \ll \omega_{+}$, the turbulent spectrum lies in the LPM regime where interactions are strongly nonlocal. Remarkably, we will see that in the limit of strongly asymmetric interactions the 3-wave kinetic equation reduces to a diffusion equation whose stationary solution is $\omega^{-2}$ regardless of the regime in which the interactions occur. This limiting case yields a mild logarithmic dependence of $\omega_{\mathrm{BH}}$ on the ultraviolet sector (see Eq. (4.60) and discussion above). The fact that the flux diverges on the $\mathrm{KZ}$ spectrum (4.30) indicates that the dominant contributions to the collision term are given by strongly asymmetric merging or branching. Therefore, one expects that in the region above the forcing scale, $\omega \gg \omega_{f}$, where the direct energy cascade forms, the integral receives a major contribution from the region $q \sim \omega_{f} \ll \omega$, while, in the opposite limit, $\omega \ll \omega_{f}$, we expect to find $q \sim \omega_{f} \gg \omega$. In what follows, we shall analyze the kinetic equation in these two regimes.

\subsection{Thermalization of the soft sector $\left(\omega \ll \omega_{f}\right)$}

Consider first the soft sector defined by $\omega \ll \omega_{f}$. Since energy is carried by a direct cascade from the source, we expect the flux to vanish below the forcing scale at late times. This can only be achieved if the spectrum 


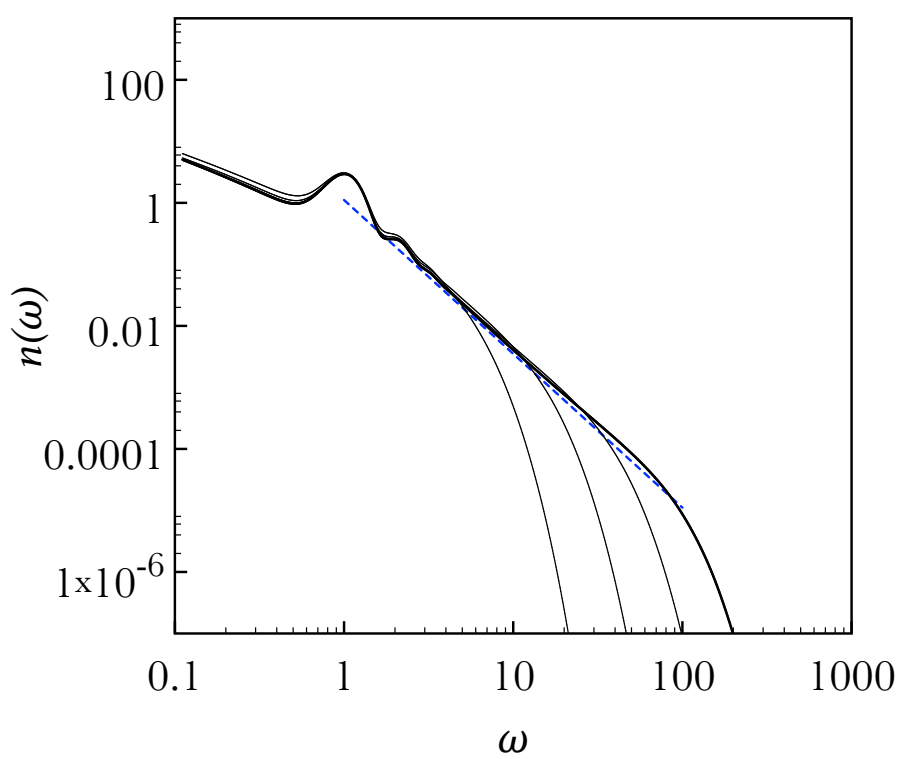

Figure 10: (Color online) Numerical simulation of the 3 -wave kinetic equation with $\beta=-1$ (characterized by local interactions). From left to right, the various curves correspond to the times $\tau=10,41,163,651$, where the rescaled time $\tau$ defined in Eq. (3.20). The dashed blue line corresponds to the local $\mathrm{KZ}$ spectrum $A_{\mathrm{KZ}} P^{1 / 2} \omega^{-5 / 2}$ with $A_{\mathrm{KZ}} \simeq 1.11$ computed using Eq. (4.32) letting $\kappa=1$ and $P_{f}=1$.

vanishes in this region or the soft sector is in thermal equilibrium, that is, $n(\omega) \sim T_{*} / \omega$. We will see that the latter is the physical solution.

Assuming the collision term to be regular in the limit $\omega \rightarrow 0$, one can neglect in Eq. (4.21) the second term, which corresponds to interactions of the mode $\omega$ with a lower mode $\omega-q$ (cf. Figure 9). Thus,

$$
I_{\text {inel }}[n] \simeq \frac{1}{\omega^{3}} \int_{0}^{\infty} \mathrm{d} q K(\omega+q, q) F(\omega+q, \omega),
$$

In the region $\omega \ll q$, the kernel (4.23) reduces to

$$
K(\omega+q, q) \simeq \kappa \frac{q^{2}}{\omega^{\beta}} .
$$


and the expansion of $F(\omega, \omega+q)$ for large $q$ yields

$$
\begin{aligned}
F(\omega+q, \omega) & \simeq n(\omega)[n(\omega+q)-n(q)]+n^{2}(q) . \\
& \simeq \omega n(\omega) \frac{\partial}{\partial q} n(q)+n^{2}(q) .
\end{aligned}
$$

Note that although the first term is proportional to $\omega$ it is enhanced by the potentially large occupation number, $n(\omega) \gg n(q)$, in the soft sector. Inserting Eqs. (4.34) and (4.35) in Eq. (4.33) yields

$$
\frac{\partial n(\omega)}{\partial t}=\frac{2 \pi^{2} \kappa I_{b}}{\omega^{2+\beta}}\left[\frac{T_{*}}{\omega}-n(\omega)\right],
$$

where we have integrated by part in the first term as follows (cf. Eq. (3.5))

$$
\int_{0}^{\infty} \mathrm{d} q q^{2} \frac{\partial}{\partial q} n(q)=-2 \int_{0}^{\infty} \mathrm{d} q q n(q)=-2 \pi^{2} I_{b},
$$

by neglecting the boundary terms and assuming that $q^{2} n(q) \rightarrow 0$ when $q \rightarrow 0$ and $q \rightarrow \infty$. Interestingly, we encounter again the effective temperature $T_{*}=I_{a} / I_{b}$ (cf. Eq. (3.9)) that appears in the Fokker-Planck equation for elastic scatterings (Eqs. (3.6) and (3.7)).

As argued above, the Bethe-Heitler scale is of the order of the forcing scale (or slightly higher). Therefore, to the left of the forcing scale the spectrum is in the BH regime and Eq. (4.36) is to be evaluated for $\beta=0$.

Now, Eq. (4.36) takes the form a Boltzmann equation in the relaxation time approximation

$$
\frac{\partial n(\omega)}{\partial t}=\frac{n_{\mathrm{eq}}(\omega)-n(\omega)}{\tau_{\mathrm{rel}}(\omega)},
$$

where the equilibrium distribution is given by the Rayleigh-Jeans distribution (2.9), with vanishing chemical potential $\mu=0$, and the relaxation time, given by

$$
\tau_{\mathrm{rel}}(\omega)=\frac{\omega^{2}}{4 \pi^{3} \bar{\alpha}^{2} C I_{a}} \sim \frac{\omega^{2}}{\hat{q}}
$$

is decreasing with $\omega$ and depends on time through $\hat{q} \equiv \hat{q}(t)$. It is of the order of the characteristic time scale for elastic processes described by the FP equation (3.4). This is a formal demonstration of the fact that elastic 
and inelastic processes are in effect comparable. This will be completed by a similar observation in the next section, which is devoted to the UV sector.

Recalling that $\tau_{\mathrm{el}}(\omega) \equiv \tau_{\mathrm{el}}(\omega, t)$ and $T_{*} \equiv T_{*}(t)$ are functions of time, the solution to Eq. (4.38) can be rewritten formally as,

$$
n(\omega)=\int_{0}^{t} \mathrm{~d} t^{\prime} \exp \left[-\int_{t^{\prime}}^{t} \frac{\mathrm{d} t^{\prime \prime}}{\tau_{\mathrm{rel}}\left(\omega, t^{\prime \prime}\right)}\right] \frac{n_{\mathrm{eq}}\left(\omega, t^{\prime}\right)}{\tau_{\mathrm{el}}\left(\omega, t^{\prime}\right)},
$$

where we have used the initial condition $n(\omega, t=0)=0$.

This solution exhibits two regimes: a radiation dominated regime at early times and a thermal regime at late times (or small $\omega$ ). In the former, the source deposits gluons at $\omega_{f}$ via radiation. Those gluons then rarely scatter or radiate so long as the occupation number is low. During this phase, the occupation number at the source grows linearly with time until nonlinear effects become important enough to saturate the distribution, this happens parametrically when $t \sim \tau_{\text {el }}\left(\omega_{f}\right)$. Hence, in this transient regime, $t \ll \tau_{\text {el }}\left(\omega_{f}\right)$, $n\left(\omega_{f}\right) \sim t$ and as a result, $\hat{q} \sim t^{2}$ and $T_{*} \sim t$. The rare scatterings induce a radiation spectrum:

$$
n(\omega) \simeq \frac{n_{\mathrm{eq}}(\omega)}{\tau_{\mathrm{rel}}(\omega)} t \sim \frac{\omega_{*}^{2}(t)}{\omega^{2}}
$$

so long as $t \ll \tau_{\text {rel }}(\omega)$, which implies $\omega_{f}>\omega \gg \omega_{*}(t) \equiv \sqrt{\hat{q} t}$. The distribution below $\omega_{*}(t)$ has already relaxed to the thermal distribution

$$
n_{\mathrm{eq}}(\omega) \equiv \frac{T_{*}}{\omega}
$$

as a result of an exact balance between the soft gluon radiation and absorption rates. To see this more clearly let us assume that $\hat{q}$ and $T_{*}$ are constants. Hence, Eq. (4.40) can be integrated to yield the simple form:

$$
n(\omega)=\left[1-\exp \left(-\frac{t}{\tau_{\mathrm{rel}}(\omega)}\right)\right] n_{\mathrm{eq}}(\omega)
$$

which clearly exhibits the two limiting cases (4.41) when $\omega \gg \omega_{*}(t)$ and (4.42) when $\omega \ll \omega_{*}(t)$. Note that the thermal distribution $n_{\text {eq }}(\omega)$ is instantaneously generated in the soft sector and propagates toward the ultraviolet with a wave front characterized by the (diffusion-like) scale $\omega_{*}(t)=\sqrt{\hat{q} t}$, that we have already encountered in the elastic case, confirmed by the numerical simulation of the kinetic equation (see Appendix $\mathrm{H}$ for details on the 
numerical procedure) with the collision integral (4.21), shown in Figure. 11. In this regime, a thermal bath forms in the region $\omega<\omega_{*}(t)$ due nonlocal interactions of the soft modes with the source. The thermalization of the soft modes have been recently thoroughly discussed in a related problem, that is, the thermalization of an isotropic plasma [28]. In this case, the hard modes present initially act effectively as a source for the soft gluons.

When $t \sim \tau_{\text {rel }}\left(\omega_{f}\right)$, the front reaches $\omega_{f}$ and the entire soft sector is in thermal equilibrium. At this point, energy starts flowing to the right of the forcing scale as a result of the excitation of higher modes. As a result, the effective temperature $T_{*}$ and the diffusion coefficient $\hat{q}$ vary slowly in time as they are mostly sensitive to the occupancy at the forcing scale, which saturates after the initial phase.

The thermalization of the soft sector in the free turbulence scenario proceeds in a similar fashion, where the initial spectrum acts as a reservoir of energy for the soft modes. The presence of elastic processes does not change this conclusion, however, the interplay between elastic and inelastic terms in the kinetic equation uniquely determines the first power correction to the thermal spectrum [28].

\subsubsection{Nonlocal turbulent spectrum for $\omega \gg \omega_{f}$}

In the previous section we have seen that after a time $t \sim \tau_{\text {el }}\left(\omega_{f}\right)$ a thermal bath forms to the left of the forcing scale. We turn now to the late time dynamics of the system, i.e., $t \gg \tau_{\text {el }}\left(\omega_{f}\right)$, in the region above $\omega_{f}$ and investigate stationary state solutions of the kinetic equation, associated with the steady transport of energy toward the ultraviolet.

As we have shown in Section 4.1 interactions on the KZ spectra are nonlocal and thus cannot be achieved. Therefore, to gain more insight on the nonlocal steady state we shall analyze the kinetic equation in the regime of strongly asymmetric gluon branching (merging). This amounts to assuming either $\omega \gg q$, or $\omega \ll q$ in the first term and $\omega \simeq q$ in the second term of (4.21).

Let us start by expanding Eq. (4.22) near $q=0$. To do so, we first 


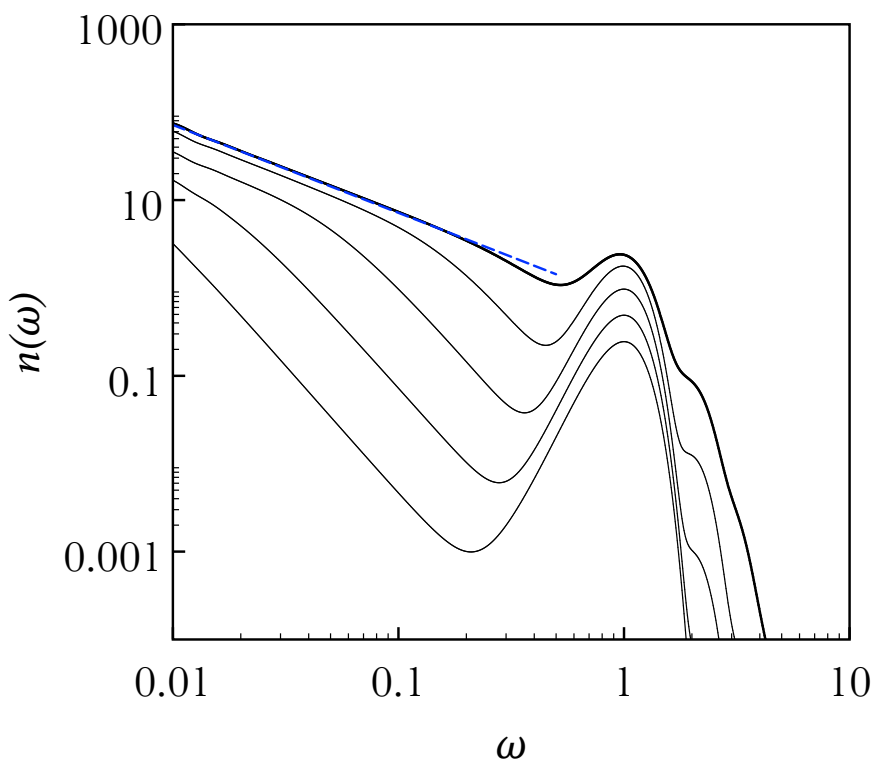

Figure 11: (Color online) Numerical solution of the kinetic equation with the inelastic collision integral given by Eq. (4.21), in the $\mathrm{BH}$ regime (for $\beta=0$ ), at early times. The occupation number is plotted at the times: $\tau=0.08$, 0.16, 0.32, 0.64 and 1.28, from bottom to top, with $\tau$ given by Eq. (3.20). The tangent (blue) dashed line corresponds to the thermal distribution $T_{*} / \omega$ with the effective $T_{*} \simeq 0.716$ evaluated at $\tau=1.28$.

multiply Eq. (4.21) by a test function $\omega^{3} g(\omega)$ and integrate over $\omega$ :

$$
\begin{aligned}
\int_{0}^{\infty} & \mathrm{d} \omega \omega^{3} g(\omega) I_{\text {inel }}[n]= \\
& -\int_{0}^{\infty} \mathrm{d} \omega \int_{0}^{\infty} \mathrm{d} q K(\omega, q) F(\omega, \omega+q)[g(\omega+q)-g(\omega)],
\end{aligned}
$$

where, we have made the substitution: $\omega \rightarrow \omega+q$, in the first term. This allows to make the following approximation,

$$
g(\omega+q)-g(\omega) \simeq q \frac{\partial}{\partial \omega} g(\omega)
$$


Integrating by part and noting that $g(\omega)$ is an arbitrary function of $\omega$, we obtain for contribution to the collision integral from the region $q \ll \omega$ (denoted by $I^{<}$),

$$
I_{\text {inel }}^{<}[n] \simeq \frac{1}{\omega^{3}} \frac{\partial}{\partial \omega} \int_{0}^{q_{\max }} \mathrm{d} q q K(\omega, q) F(\omega+q, q),
$$

where by consistency the $q$ integration must be cutoff at $q_{\max }<\omega$.

Assuming that the distribution $n(\omega)$ is steeper than $1 / \omega$ one can neglect the first term in $F(\omega, \omega+q)$ (cf. Eq. (4.22)), and thus, one can write

$$
F(\omega, \omega+q) \simeq[n(\omega+q)-n(\omega)] n(q) \simeq q n(q) \frac{\partial}{\partial \omega} n(\omega) .
$$

In this approximation, the kernel (4.23) reduces to

$$
K(\omega, q) \simeq \kappa \frac{\omega^{3}}{q^{\beta+1}}
$$

Inserting Eqs. (4.47) and (4.48) in the collision term (4.46), we obtain a the following diffusion equation

$$
I_{\text {inel }}^{<}[n]=\frac{1}{4 \omega^{3}} \frac{\partial}{\partial \omega} \omega^{3} \hat{q}_{\text {inel }} \frac{\partial}{\partial \omega} n(\omega),
$$

where the diffusion coefficient reads $\hat{q}_{\text {inel }} \equiv 4 \pi^{2} \kappa I_{c}$, with

$$
I_{c} \equiv \frac{1}{\pi^{2}} \int_{0}^{q_{\max }} \mathrm{d} q q^{1-\beta} n(q),
$$

that reduces to $I_{b}$ in the $\mathrm{BH}$ regime, i.e. when $\beta=0$.

Note that Eq. (4.49) has two fixed points $n(\omega)=$ const. and $n(\omega)=\omega^{-2}$, which are independent of $\beta$. The second solution is the one related to constant flux of energy $P$ carried by a direct energy cascade. For a power spectrum that is steeper than $q^{-2+\beta}, I_{c}$ is dominated by the lower bound, thus, in the LPM regime, where $\beta=1 / 2$ one can safely send $q_{\max }$ to infinity. On the other hand, in the $\mathrm{BH}$ regime, $I_{c}=I_{b}$ is only logarithmically dependent on $q_{\max }$ which should not affect any power like behavior and, similarly to the Coulomb logarithm in the elastic collision integral, may be ignored. More generally, we can write

$$
\hat{q}_{\text {inel }}=\frac{4 \kappa}{\omega^{3}} \int_{0}^{\infty} \mathrm{d} q q^{2} K(\omega, q) n(q) .
$$


Interestingly, Eq. (4.49) describes a diffusion process in four dimensions as a consequence of energy conservation. This is in contrast with three dimensional diffusion whose integral of motion is the total particle number.

Likewise, the contribution from $q \gg \omega$ in the first term of Eq. (4.22) yield,

$$
\frac{1}{\omega^{3}} \int_{q_{\min }}^{\infty} \mathrm{d} q q K(\omega, q) F(\omega+q, q) \simeq \frac{\kappa}{\omega^{2+\beta}} \int_{q_{\min }}^{\infty} \mathrm{d} q q^{2} \frac{\partial n(q)}{\partial q} n(\omega)
$$

where we have neglected the term $n^{2}(q)$ in $F(\omega+q, \omega)$ assuming that the spectrum is steeper than $\omega^{-1}$. Here, we recover the integral $I_{b}$ (cf. Eq. 3.5), which is dominated by the UV when the spectra exponent of the distribution $n(q)$ is larger than -2 the integral $I_{b}$, in which case, one can set the lower bound to 0 .

We consider now the region $q \simeq \omega$ in the second term and make the change of variables $q \rightarrow \omega-q$. We obtain

$$
\begin{aligned}
\frac{1}{\omega^{3}} \int_{0}^{\omega} \mathrm{d} q q K(\omega-q, q) F(\omega, q) & =\frac{1}{\omega^{3}} \int_{0}^{\omega} \mathrm{d} q q K(q, \omega-q) F(\omega, \omega-q) \\
& \simeq \frac{1}{\omega} \int_{0}^{q_{\max }} \mathrm{d} q q^{1-\beta} n(q) \frac{\partial n(\omega)}{\partial \omega} \\
& \simeq \frac{\hat{q}_{\text {inel }}}{4 \omega} \frac{\partial n(\omega)}{\partial \omega}
\end{aligned}
$$

where $\hat{q}_{\text {inel }}$ is related to the integral $I_{c}$ (cf. Eq. (4.50) and above) that we already encountered when analyzing the region $q \ll \omega$.

Putting all the pieces together and assuming $\hat{q}_{\text {inel }}$ to be independent of $\omega$, the inelastic collision integral reads

$$
\frac{\partial n(\omega)}{\partial t} \simeq \frac{\hat{q}_{\text {inel }}}{4 \omega^{3}} \frac{\partial}{\partial \omega} \omega^{3} \frac{\partial}{\partial \omega} n(\omega)-\frac{\hat{q}_{\text {inel }}}{4 \omega} \frac{\partial n(\omega)}{\partial \omega}+\frac{2 \pi^{2} \kappa}{\omega^{2+\beta}} I_{b} n(\omega)
$$

It is straightforward to verify that the first diffusive term and the last two terms conserve energy separately. Close to the ultraviolet cutoff the last term is suppressed since it accounts for interactions of the mode $\omega$ with very hard modes. In this case the first two terms combine to yield the diffusion 
equation

$$
\frac{\partial n(\omega)}{\partial t} \simeq \frac{\hat{q}_{\text {inel }}}{4 \omega^{2}} \frac{\partial}{\partial \omega} \omega^{2} \frac{\partial}{\partial \omega} n(\omega)
$$

It follows that a diffusive process is responsible for the energy transport toward to UV. Hence, we expect the UV cutoff to scale as $t^{1 / 2}$. This will be checked soon in numerical simulations of the complete inelastic collision term.

Because the collision term Eq. (4.54) is inhomogeneous in $\omega$ no steady power law solution is expected. Of course, close to the UV cutoff, one can neglect the third term, in which case, the power spectrum $\omega^{-1}$ becomes a steady state solution Eq. (4.54). But since this solution is related to the conservation of particle number through the diffusion term given by Eq. (4.55) it cannot be associated with the direct energy cascade.

In Figure 12, the numerical solution of Eq. (4.54) is plotted for various times. First, we note that the power exponent is close to -2 . However, the spectrum is not steady in the wave of the right moving front due to the presence of the last two terms in the r.h.s. of Eq. (4.54) that are responsible for the slow decrease of the spectrum as a function of time. The red(dotted) curve corresponds to the analytic solution of Eq. (4.54) where only the first diffusive term is kept (cf. Eq. (4.56)).

To gain more insight on the scaling of the turbulent spectrum in the inertial range let us consider a simplified equation. Let us only keep the first energy conserving term in Eq. (4.54). Away from the source, namely when $\omega \gg \omega_{f}$, the solution reads (see details in Appendix G)

$$
n(\omega) \simeq \frac{4 \pi^{2} P_{f}}{\hat{q}_{\text {inel }} \omega^{2}} \exp \left(-\frac{\omega^{2}}{\hat{q}_{\text {inel }} t}\right),
$$

which describes a quasi-steady spectrum $\omega^{-2}$ at late times, provided $\hat{q}_{\text {inel }}$ is a slowly varying function of time, which will be shortly verified. This spectrum forms in the wake of a diffusive front characterized by the scale $\omega_{*}(t)=\sqrt{\hat{q}_{\text {inel }} t}>\omega_{f}$. It is associated with a direct energy cascade that carries a constant energy flux toward growing $\omega$ 's.

By matching the solutions to the left and to the right of the forcing scale, parametrically, we have $n\left(\omega_{f}\right) \sim T_{*} / \omega_{f} \sim P_{f} / \hat{q}_{\text {inel }} \omega_{f}^{2}$. This implies that $T_{*} \sim P_{f} / \hat{q}_{\text {inel }} \omega_{f}$. Moreover, using the forms of the spectrum for $\omega<\omega_{f}$, 


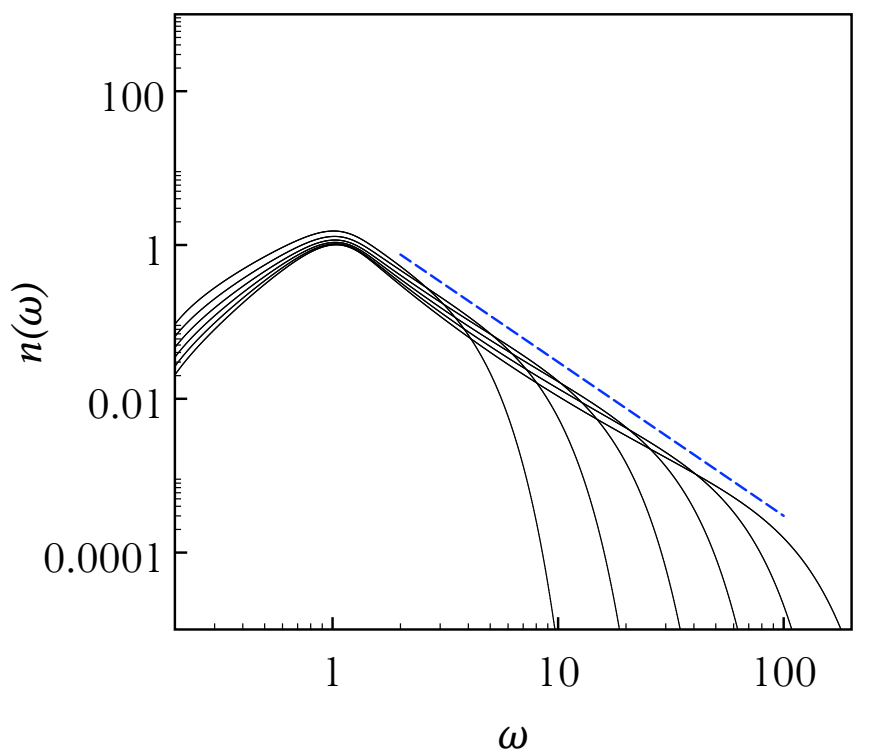

Figure 12: (Color online) Numerical solution of Eq. (4.54) for $\kappa=1$ at $t=1$, 4, 16, 64, 256 and 1000 (from left to right) in the LPM regimes, i.e. $\beta=1 / 2$. The (blue) dashed line corresponds to the power law $\omega^{-2}$ and is given as reference.

and $\omega>\omega_{f}$, namely, $\omega^{-1}$ and $\omega^{-2}$, respectively, we observe that the modes that contribute to the diffusion coefficient are of order $q \sim \omega_{f}$. Hence, the diffusion coefficient scales as $\hat{q} \sim \bar{\alpha}^{2} \omega_{f}^{3} n^{2}\left(\omega_{f}\right)$.

On the other hand, the inelastic diffusion coefficient is also dominated by the forcing scale, which is consistent with the nonlocal nature of the interactions justifying the gradient expansion. In the LPM regime we find

$$
\hat{q}_{\text {inel }} \simeq 4 \bar{\alpha} \sqrt{\hat{q}} \int \mathrm{d} q q^{1 / 2} n^{2}(q) \sim \bar{\alpha}^{2} \omega_{f}^{3} n^{2}\left(\omega_{f}\right) .
$$

Using $T_{*} \sim P_{f} / \hat{q}_{\text {inel }} \omega_{f}$ together with Eq. (4.57) yield

$$
\hat{q}_{\text {inel }} \sim \hat{q} \sim \bar{\alpha}^{2 / 3} P_{f}^{2 / 3} \omega_{f}^{-1 / 3}, \quad \text { and } \quad T_{*} \sim \bar{\alpha}^{-2 / 3} P_{f}^{1 / 3} \omega_{f}^{-2 / 3} .
$$

This parametric estimate was derived in the LPM regime, but it can be easily checked that it holds in the $\mathrm{BH}$ regime too. 
Finally, inserting Eq. (4.58) in Eq. (4.56) for $\omega_{f} \ll \omega \ll \omega_{*}(t)$, i.e., in the inertial range, we find that the steady state spectrum associated with a constant flux reads

$$
n(\omega) \sim \frac{A}{\omega^{2}}, \quad \text { with } \quad A \sim \frac{P_{f}^{1 / 3} \omega_{f}^{1 / 3}}{\bar{\alpha}^{2 / 3}} .
$$

Note that, as anticipated in the introduction, the steady state spectrum for quasi-collinear (inelastic) 1 to 2 processes, scales as $P_{f}^{1 / 3}$ similarly to 2 to 2 processes (cf. Eq. (3.23)). Moreover, there is an explicit dependence on the forcing scale $\omega_{f}$ demonstrating the nonlocality of inelastic interactions in a turbulent state.

In the above analysis, we have neglected the contributions of democratic splittings to the collision integral and concluded that energy is transport by diffusion toward the UV. To assess the validity of this approximation we have computed numerically the occupation number at late times in the $\mathrm{BH}$ and LPM regimes. This is shown in Figure 13. In the BH regime a clear stationary spectrum with spectral exponent -2 forms in the wake of the right moving front.

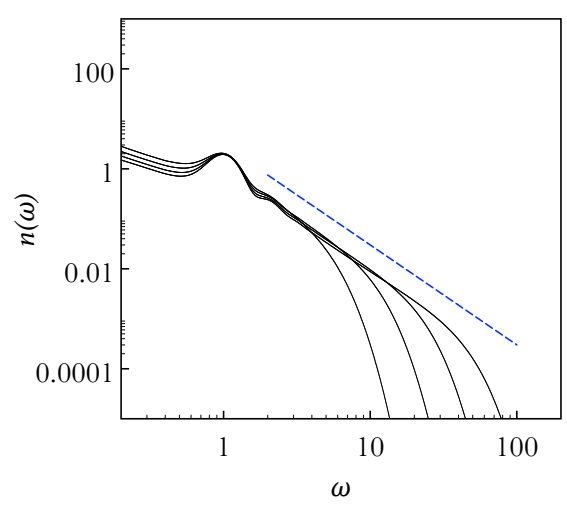

(a)

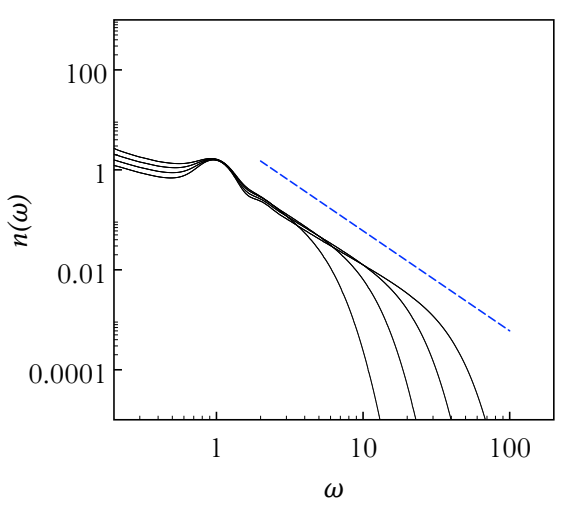

(b)

Figure 13: (Color online) The gluon spectrum in the pure inelastic case for $\kappa=1$ at $t=1,4,16$ and 64 (from left to right) in the $\mathrm{BH}$ (left panel) and the LPM regimes (right panel), respectively. The (blue) dashed line corresponds to the power law $\omega^{-2}$ and is given for reference. 


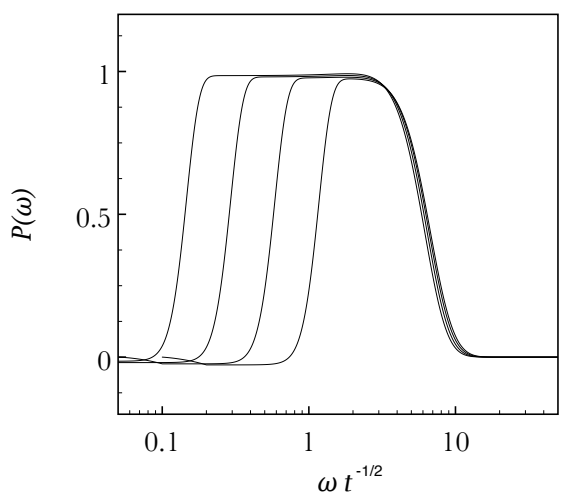

(a)

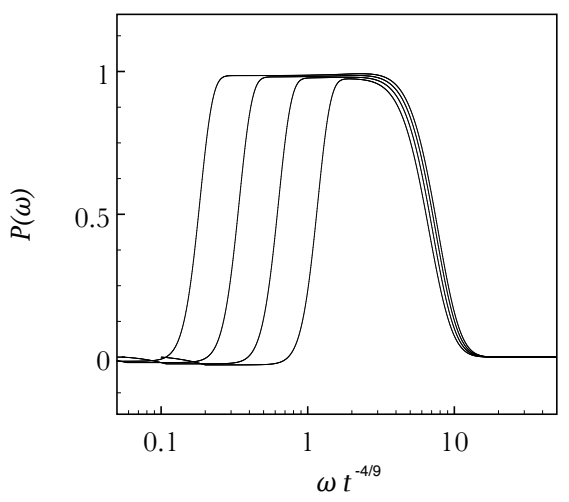

(b)

Figure 14: (Color online) The energy flux density as a function of the rescaled momentum $\omega t^{-1 / 2}$ (left panel) and $\omega t^{-4 / 9}$ (right panel) in the LPM regime. From left to right: $t=1,4,16$ and 64 .

Incidentally, the exponent -2 is also that of the KZ spectrum established in Section 4.1, which we argued was only marginally nonlocal and hence, could be realized with a mild logarithmic corrections. However, no evidence of this logarithmic dependence of the spectrum was found is the numerical study.

In Figure 13(b), we have plotted the spectrum at various times in the LPM regime. We observe that as the front moves to the right the spectrum slowly decreases in its wake, in particular near the source. This is the result of nonlocal interactions that flatten the spectrum as the time goes on.

If the LPM regime were dominated by local interactions one should expect the front to scale as $t^{9 / 4}$, as shown in the next section. However, Figure 14, where the energy flux is plotted, shows that the wave front scales as $t^{1 / 2}$, rather than the unphysical local scaling $t^{9 / 4}$. Indeed, the former scaling implies a faster wave propagation and therefore, nonlocal interactions are more efficient in transporting energy to the UV. Similarly, the wave front in the $\mathrm{BH}$ regime is also found to scale as $t^{1 / 2}$.

The above analysis is valid as long as nonlocal interactions are dominated by exchanges with the source, that is, when the integral $I_{c}$ is dominated by the IR. This assumption must be relaxed when the spectrum exponent is 
larger that $-2+\beta=-3 / 2$. In this case, the interactions will be dominated by exchanges with the UV end of the spectrum and source would playing a marginal role. I this case, according to the conclusion in the previous section, the spectrum must be in thermal equilibrium with the UV that plays now the role of the source.

For completeness, we shall evaluate the $\mathrm{BH}$ scale in order to estimate the relevance of either of the regimes in the inertial range and in the soft sector. The integral $I_{a}$ (cf. Eq. (3.5)) is dominated by momenta of the order of the forcing scale, $I_{a} \sim \int \mathrm{d} q q^{2} n^{2}(q) \sim A^{2} \omega_{f}$, whereas, the integral $I_{b}$ evaluated for the turbulent spectrum Eq. (4.56) yields a logarithmic dependence on the ultraviolet cutoff $\omega_{*}(t) \gg \omega_{f}, I_{b} \sim A \ln \omega_{*}(t) / \omega_{f}$. Using these estimates in Eq. (4.6), we find that

$$
\omega_{\mathrm{BH}} \sim \omega_{f} \ln ^{2} \frac{\omega_{*}(t)}{\omega_{f}} .
$$

Apart from the mild logarithmically increase, $\omega_{\mathrm{BH}}$ is of the order of the forcing scale $\omega_{f}$. Since hard modes in the inertial range interact mainly with modes near $\omega_{f}$, one may argue that the $\mathrm{BH}$ branching rate is a better approximation of the full kernel, which obeys Eq. (4.7).

Similarly, using Eq. (4.59) in Eq. (1.4), we find that the Debye mass scales parametrically as

$$
m_{D}^{2} \sim \bar{\alpha}^{1 / 3} P_{f}^{1 / 3} \omega_{f}^{1 / 3} \ln \frac{\omega_{*}(t)}{\omega_{f}},
$$

which must be much smaller than $\omega_{f}$ for this analysis, based on neglecting the gluon mass dependence in the dispersion relation and the small angle approximation, to be valid.

These estimates must be reevaluated when the spectrum flattens toward the thermal one at late times. In this situation, the integrals $I_{a}$ and $I_{b}$ will be dominated by the UV cutoff, and we obtain $\omega_{\mathrm{BH}} \simeq \omega_{*}(t)$, while the Debye mass reads $m_{D}^{2} \sim \bar{\alpha} T_{*} \omega_{*}(t)$ for $n \sim T_{*} / \omega$, and remains suppressed at small coupling.

\subsubsection{Analytic form of the front: $\omega \gg \omega_{*}(t)$}

The merging of gluons of comparable energies, which is not taken into account in the diffusion approximation, is the dominant process in the region of the wave front, namely when $\omega \gg \omega_{*}(t)$ and yields an exponential 
suppression rather than Gaussian, as shown in Eq. (4.56). To see this, note that this regime corresponds to the edge of the phase space, where a gluon $\omega$ is dominantly produced by interactions with lower bins (merging). This amounts in neglecting the first term in Eq. (4.13). Moreover, due to the stiffness of the spectrum, $n(\omega) \ll n(q) \sim n(\omega-q)$, and one can neglect terms promotional to $n(\omega)$. It follows that $F(\omega-q, \omega) \approx-n(\omega-q) n(q)$ and Eq. (4.13) reduces to

$$
\frac{\partial}{\partial t} n(\omega) \approx \frac{1}{\omega^{3}} \int_{0}^{\omega} \mathrm{d} q K(\omega, q) n(\omega-q) n(q) .
$$

Before going on solving Eq. (4.62), let us first determine the speed of the front by assuming a self-similar evolution of the occupation number,

$$
n(\omega) \equiv t^{a} f\left(\omega t^{b}\right)
$$

where $a$ and $b$ will be fixed uniquely by energy conservation and the homogeneity of the kinetic equation. Then, using the kernel form (4.23) where $\kappa$ is roughly constant as a function of $\hat{q}$, Eq. (4.62) yields

$$
\begin{aligned}
t^{a-1}\left[a f(x)+b x f^{\prime}(x)\right] & =\kappa t^{2 a+\beta b} x^{-\beta} \\
& \times \int_{0}^{1} \mathrm{~d} z z^{-1-\beta}(1-z)^{-\beta} f(z x) f((1-z) x),
\end{aligned}
$$

where $x=\omega t^{b}$. The homogeneity condition implies that

$$
a+\beta b+1=0
$$

Since most of the energy is carried by the hard sector a second equation can be obtained requiring energy conservation

$$
E=P_{f} t \sim \int \mathrm{d} \omega \omega^{3} n(\omega)=t^{a-4 b} \int \mathrm{d} x x^{3} f(x),
$$

from which we must have

$$
a-4 b-1=0 .
$$

Solving Eqs. (4.65) and (4.67) for $a$ and $b$, one finds

$$
a=-\frac{4-\beta}{4+\beta}, \quad b=-\frac{2}{4+\beta},
$$


In the $\mathrm{BH}$ regime, $\beta=0$, we have

$$
a=-1, \quad b=-\frac{1}{2} .
$$

This result is indistinguishable with that of the previous section, where we have found that in the diffusion approximation the position of the front propagates as $t^{1 / 2}$. However, in the LPM regime, where $\beta=1 / 2$, one obtains a slower propagation of the front,

$$
a=-\frac{7}{9} \approx-0.78 \quad b=-\frac{4}{9} \approx-0.44 .
$$

One may argue that the mismatch between the time evolution of characteristic scales in the diffusion regime, $t^{1 / 2}$, and in the wave front, $t^{9 / 4}$, would generate an accumulation of particles in the wake of the wave front, which may cause the slope to be smaller than the predicted power -2 , as observed in the numerical simulation given in Figure 13.

Owing to the form of the r.h.s. of Eq. (4.64), we assume the following ansatz for the wave front scaling function

$$
f(x)=A x^{\gamma} \mathrm{e}^{-B x} .
$$

Injecting (4.71) in Eq. (4.64) we obtain

$$
(a+b \gamma) x^{\gamma}-B b x^{\gamma+1}=\kappa A x^{2 \gamma-\beta} \int_{0}^{1} \mathrm{~d} z z^{\gamma-1-\beta}(1-z)^{\gamma-\beta} .
$$

For the $z$ integral to converge one must have $\gamma>\beta$, and because $\beta>0$, when $x \gg 1$, one can neglect the first term in the l.h.s. of Eq. (4.72). By matching the exponents of $x$ in the second term with the r.h.s. we find that $\gamma=1+\beta$. As a result, the $z$ integral is independent of $\beta$ and yields $\int_{0}^{1} \mathrm{~d} z(1-z)=1 / 2$. Finally, solving for $A$, one gets $A=2 B /[(4+\beta) \kappa]$, and the solution for the wave front takes the form

$$
n(\omega) \simeq \frac{2 B t^{-\frac{4-\beta}{4+\beta}}}{(4+\beta) \kappa}\left(\frac{\omega}{\omega_{\beta}(t)}\right)^{1+\beta} \exp \left[-B\left(\frac{\omega}{\omega_{\beta}(t)}\right)\right] .
$$

where $\omega_{\beta}(t) \sim t^{\frac{2}{4+\beta}}$.

As shown in the previous section, numerical simulations of the kinetic equation in the LPM regime, where $\beta=1 / 2$, show that the wave front propagates faster than $t^{4 / 9}$ and is more compatible with $\omega_{*}(t) \sim t^{1 / 2}$, which characterizes the nonlocal solution (4.56). 


\section{Turbulent spectrum in the presence of elastic and inelastic pro- cesses}

Up to now, we have discussed elastic and inelastic processes separately and shown that they result in different power spectra, however, in both cases the energy cascade is found to be governed by a diffusion like transport toward the ultraviolet.

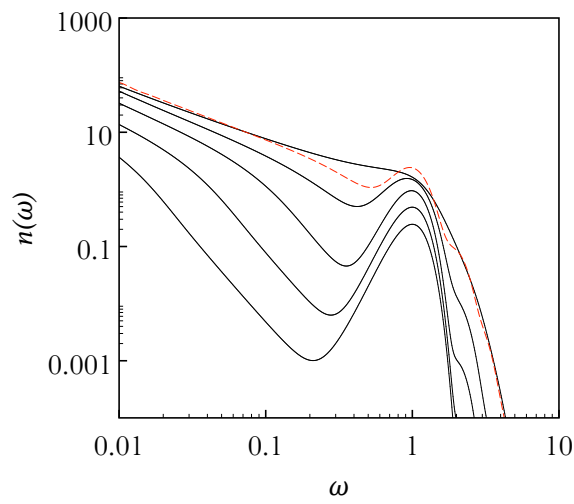

(a)

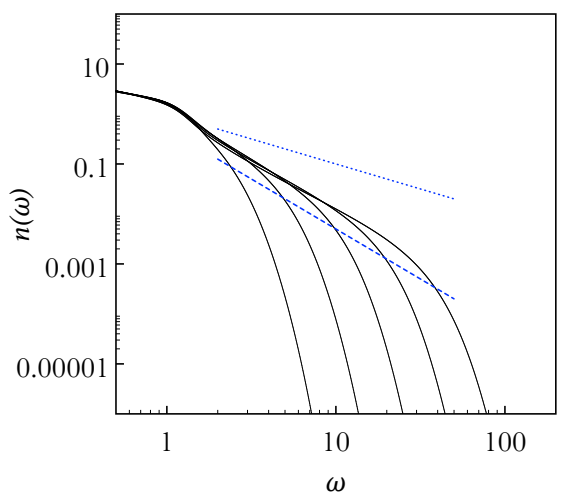

(b)

Figure 15: (Color online) The numerical solution of the full kinetic equation, with elastic and inelastic processes taken into account. Left: black curves are for $\tau=0.08,0.16,0.32,0.64$ and 1.28, from bottom to top. The dashed (red) curve shows the result of inelastic processes alone in the $\mathrm{BH}$ regime plotted at $\tau=1.28$ (to be compared to the corresponding black curve). Right: Same as in the left panel for $\tau=2,8,32,128,512$ (left to right). The dashed and dotted (blue) curves stand for the power spectra $\omega^{-2}$ and $\omega^{-1}$ and are shown as reference.

Before we turn to the direct energy cascade, let us first analyze the soft sector (to the left of the forcing scale). While in the presence of elastic processes alone we have seen that the steady state corresponds to a nonthermal distribution $A / \omega$, with $A>T_{*}$, associated with a constant flux of particles, we expect the inelastic term to prevent such a spectrum due to the singular behavior of the radiation rate in the infrared that is tamed only when the soft sector is in thermal equilibrium, regardless of the presence of the elastic term. In Figure 15 (a), we see that at early times gluons are 


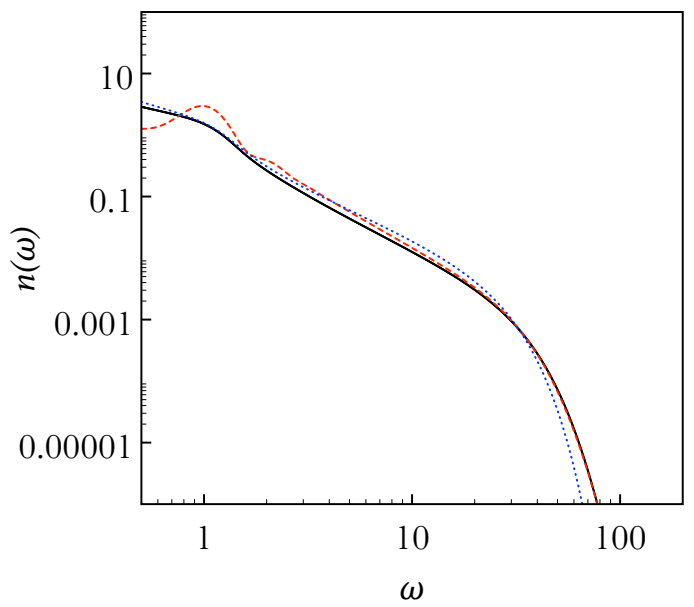

Figure 16: (Color online) The full spectrum at $\tau=512$ (full line) compared to the those in the purely elastic and inelastic cases, which are depicted by dotted (blue) and dashed (red) curves, respectively.

injected into the soft sector via radiation then a thermal spectrum develops from the left to the right with temperature $T_{*}$. The similarity with the inelastic alone is highlighted by comparing the largest time $\tau=1.28$ (full thick curve) with the result obtained with only the inelastic term taken into account (dashed red curve) and we can see that both are in semi-quantitative agreement. Hence, we conclude that inelastic processes dominate over elastic ones.

A more detailed analysis of the interplay of elastic and inelastic processes in given in Ref. [28], where a universal power correction to the thermal spectrum is derived,

$$
n(\omega) \simeq \frac{T_{*}}{\omega}+A \omega^{\alpha}, \quad \text { with } \quad \alpha=\frac{-3+\sqrt{1+4 C}}{2},
$$

where for $C \simeq 1.83$ (cf. Eq. (4.11)) we have $\alpha \simeq 0.4$, which qualitatively accounts for the deviation of the complete kinetic equation from the pure inelastic case as shown in Figure 15 (a).

This feature is not obvious since one could expect, if inelastic processes were negligible, particle number to be approximately conserved causing a finite flux of particle to develop in the infrared and thus, the formation of a 
transient Bose-Einstein condensate.

Let us turn now to the ultraviolet sector where the direct energy cascade develops. Due to nonlocal interactions there is no steady power spectrum. However, since the thermal-like spectrum $\omega^{-1}$ is a fixed point of the full kinetic equation, one may expect at very late times, the system to be driven toward the thermal fixed point with a small correction that would account for finite energy flux as argued in Ref. [11]. This is known as "warm turbulence" in the literature. When the spectral exponent becomes larger than $-3 / 2$, nonlocal interactions that were initially dominated by interactions with the source are now dominated by interactions with modes located at the UV cutoff $\omega_{*}(t)$. This is reflected by the fact that the global integrals $I_{a}, I_{b}$ and $I_{c}$ cease to be dominated by the source scale, $\omega_{f}$. In this case, the UV modes play the role of the source with which softer modes must be in thermal equilibrium.

The result of the numerical simulation for relatively late times is shown in Figure 15 (b) where the slope of the spectrum appears to be more compatible with the power -2 than the thermal one. The simulations for the purely elastic and inelastic cases exhibits a similar behavior as shown in Figure 16. However, in the region of the front wave the result for inelastic scatterings alone is closer to the full result than the purely elastic case. This may indicate that inelastic processes are dominant in transporting energy toward the UV.

\section{Summary}

In this paper, we have investigated analytically and numerically, in the framework of kinetic theory, the spectrum of gluons that forms at late times in the presence of a steady and spectrally narrow source of energy. Both elastic and inelastic processes are taken into account in the small angle approximation allowing for a semi-analytical treatment. Owing to the different nature of the two contributions, we have first discussed them separately. Elastic processes are described by a Fokker-Planck equation that conserves energy and particle number, for which non-thermal stationary solutions are derived. Below the forcing scale a inverse particle cascade characterized by the spectrum $\omega^{-1}$ develops, no finite steady spectrum is associated with the direct energy cascade. This results strongly differs from scaler $\phi^{4}$ theory, where KZ turbulent spectra are expected to form, because of the strong nonlocal nature of interactions in momentum space in Yang-Mills theory as argued 
in ??. Deep in the infrared regime, below the forcing scale, the dynamics is governed by scattering of the soft modes off gluons at the forcing scale.

We have shown that the inelastic processes are also strongly nonlocal, hence, the corresponding KZ spectra, are not physically relevant except in the $\mathrm{BH}$ regime where the $\mathrm{KZ}$ spectra $\omega^{-2}$ yields a logarithmic divergent flux and therefore, is marginally nonlocal. Below the forcing scale at late times, radiation and absorption of soft gluons by gluons at the forcing scales are exactly balanced. As a result the soft sector is in thermal equilibrium at late times. To the right (above) the forcing scale, assuming strongly nonlocal (asymmetric) interactions the kinetic equation describing the effective 1 to 2 scattering processes reduces to a diffusion-like equation that conserves energy. As a result, energy is transported to the UV by diffusion and therefore, the spectrum spreads as $t^{1 / 2}$.

Numerical simulations of the inelastic collision integral in the LPM regime agree qualitatively with the results obtained in the nonlocal approximation. In particular, the wave front is shown to scale as $t^{1 / 2}$ which is consistent with the aforementioned diffusion-like energy transport that characterizes nonlocal inelastic processes. In the inertial range, there is no steady power law spectrum due to the sensitivity of the spectrum to the UV cutoff.

Combining elastic and inelastic processes, which are qualitatively similar, one expects the spectrum to evolve toward the thermal fixed point. However, in the numerical simulations of the kinetic equation the power spectrum appears to be close to $\omega^{-2}$ which is most likely a transient power spectrum. This result, based on kinetic theory, agrees with the turbulent spectrum observed in the context of Chromo-Weibel Instabilities [8].

Finally, the above analysis breaks down when quantum effects can no longer be neglected, namely, when $n\left(\omega_{*}\right) \sim 1$, forcing the spectrum to evolve toward the Bose-Einstein distribution.

In summary, nonlocal interactions are dominant in non-Abelian gauge plasma. Thus, the resulting turbulent spectrum is not universal and depends strongly on the physics at the forcing and dissipation scales. We have shown that the spectrum in the presence of elastic and inelastic processes flattens when the time goes on and hence is not steady. However, further investigation are necessary in order to better understand the late time dynamics of the turbulent spectrum in non-Abelian theories.

\section{Acknowledgements}

We would like the thank J.-P. Blaizot, D. B. Kaplan, J. Liao, A. Kurkela, A. H. Mueller, M. Strickland and K. Tywoniuk for many discussions, and 
in particular J.-P. Blaizot and K. Tywoniuk for the careful reading of the manuscript. We would also like to thank J. Liao and J.-P. Blaizot for fruitful discussions and collaboration on issues related to this work. This research is supported by the U.S. Department of Energy under Contract No. DE-FG0200ER41132.

\section{Appendix A. Zakharov conformal transformations}

In this appendix we derive the Zakharov transformation that make the power-like non-thermal fixed points of the collision integral explicit. After integrating over $\omega_{1}$ with the help of the delta function in Eq. (2.10), the remaining double integration over $\omega_{2}$ and $\omega_{3}$ is to be performed over the region $\Delta$ defined by $\omega_{2}>0, \omega_{3}>0$ and $\omega_{2}+\omega_{3}>\omega$, with $\omega_{1}+\omega=\omega_{2}+\omega_{3}$ from energy conservation. It is useful to divide $\Delta$ into four subregions of integration as shown in Figure (2). Each integration region corresponds to the various possible orderings of $\omega$ and the other three frequencies. In Region 1 , defined by

$$
\Delta_{1} \equiv\left\{\omega>\omega_{2}>0, \omega>\omega_{3}>\omega-\omega_{2}\right\},
$$

where the largest frequency of the quartet is $\omega$. Likewise, for region 2, 3 and 4 , we have respectively

$$
\begin{aligned}
& \Delta_{2} \equiv\left\{\omega>\omega_{2}>0, \omega_{3}>\omega\right\}, \text { with } \max \left(\omega, \omega_{1}, \omega_{2}, \omega_{3}\right)=\omega_{3},(A), \text { with } \max \left(\omega, \omega_{1}, \omega_{2}, \omega_{3}\right)=\omega_{1}, \\
& \Delta_{3} \equiv\left\{\omega_{2}>\omega, \omega_{3}>\omega\right\}, \quad \text { with } \max \left(\omega, \omega_{1}, \omega_{2}, \omega_{3}\right)=\omega_{2} . \\
& \Delta_{4} \equiv\left\{\omega>\omega_{2}>0, \omega_{3}>\omega\right\},
\end{aligned}
$$

Now, we look for a conformal transformation for each region that will transform the largest frequency of the quartet into $\omega$. Consider for instance Region 2. We seek a transformation $\left(\omega, \omega_{1}, \omega_{2}, \omega_{3}\right) \rightarrow\left(\omega^{\prime}, \omega_{1}^{\prime}, \omega_{2}^{\prime}, \omega_{3}^{\prime}\right)$, that preserves the constraint $\omega+\omega_{1}-\omega_{2}-\omega_{3}=\omega+\omega_{1}^{\prime}-\omega_{2}^{\prime}-\omega_{3}^{\prime}=0$, such that

$$
\omega_{2}<\omega\left(\omega_{1}\right)<\omega_{3} \rightarrow \omega_{1}^{\prime}<\omega_{3}^{\prime}\left(\omega_{2}^{\prime}\right)<\omega .
$$

To do so, we multiply the quartet by a scale factor $\lambda$ such that ${ }^{8}$

$$
\left(\lambda \omega, \lambda \omega_{1}, \lambda \omega_{2}, \lambda \omega_{3}\right)=\left(\omega_{3}^{\prime}, \omega_{2}^{\prime}, \omega_{1}^{\prime}, \omega^{\prime}\right) .
$$

\footnotetext{
${ }^{8}$ There exists two possible transformations owing to the symmetry in the exchange of $\omega_{2}$ and $\omega_{3}$.
} 
Of course, since $\omega$ is the only non-dummy variable we require $\omega^{\prime}=\omega$. Eqs. (A.5) and (A.6) imply that $\lambda=\omega / \omega_{3}=\omega_{3}^{\prime} / \omega$, thereby, we can readily identify

$$
\omega_{1}^{\prime}=\frac{\omega \omega_{2}}{\omega_{3}}, \quad \omega_{2}^{\prime}=\frac{\omega \omega_{1}}{\omega_{3}}, \quad \omega_{3}^{\prime}=\frac{\omega^{2}}{\omega_{3}} .
$$

Let us now perform the transformation (A.7) on the corresponding term in the collision integral. First, the integration measure transforms as follows

$$
\mathrm{d} \omega_{3} \mathrm{~d} \omega_{2}=\mathrm{d} \omega_{3}^{\prime} \mathrm{d} \omega_{2}^{\prime}\left(\frac{\omega^{\prime}}{\omega_{3}^{\prime}}\right)^{3}
$$

The homogeneous function $U$ (cf. Eq. (2.11)), yields

$$
U\left(\omega, \omega_{1}, \omega_{2}, \omega_{3}\right)=\lambda^{-\nu} U\left(\lambda \omega, \lambda \omega_{1}, \lambda \omega_{2}, \lambda \omega_{3}\right)=\left(\frac{\omega}{\omega_{3}^{\prime}}\right)^{\nu} U\left(\omega_{3}^{\prime}, \omega_{2}^{\prime}, \omega_{1}^{\prime}, \omega\right)
$$

Similarly, for the factor $F[n] \equiv F\left(\omega, \omega_{1}, \omega_{2}, \omega_{3}\right)$, containing the occupation numbers, we obtain

$$
\begin{aligned}
F\left(\omega, \omega_{1}, \omega_{2}, \omega_{3}\right) & =\left(\frac{\omega_{3}^{\prime}}{\omega}\right)^{-3 x} F\left(\omega_{3}^{\prime}, \omega_{2}^{\prime}, \omega_{1}^{\prime}, \omega\right) \\
& =-\left(\frac{\omega_{3}^{\prime}}{\omega}\right)^{-3 x} F\left(\omega, \omega_{1}^{\prime}, \omega_{2}^{\prime}, \omega_{3}^{\prime}\right)
\end{aligned}
$$

where in last equality we have used the symmetry properties of $F[n]$, namely,

$$
\begin{aligned}
F\left(\omega, \omega_{1}, \omega_{2}, \omega_{3}\right) & =F\left(\omega_{1}, \omega, \omega_{2}, \omega_{3}\right)=F\left(\omega, \omega_{1}, \omega_{3}, \omega_{2}\right) \\
& =-F\left(\omega, \omega_{1}, \omega_{2}, \omega_{3}\right) .
\end{aligned}
$$

Finally, the result of Zakharov transformation on Region 2 reads

$$
-\frac{g^{2}}{2(4 \pi)^{3}} \int_{\Delta_{1}} \mathrm{~d} \omega_{2} \mathrm{~d} \omega_{3} \frac{\omega_{1}}{\omega^{2}}\left(\frac{\omega_{3}}{\omega}\right)^{-3 x-4} F\left(\omega, \omega_{1}, \omega_{2}, \omega_{3}\right) .
$$

where we have relabelled the dummy variables $\omega_{2}^{\prime}$ and $\omega_{3}^{\prime}$ as $\omega_{2}$ and $\omega_{3}$. 
Following the same reasoning regions 3 and 4 are mapped into region 1 , using the conformal transformations,

$$
\omega_{1}^{\prime}=\frac{\omega^{2}}{\omega_{1}}, \quad \omega_{2}^{\prime}=\frac{\omega \omega_{2}}{\omega_{1}}, \quad \omega_{3}^{\prime}=\frac{\omega \omega_{3}}{\omega_{1}},
$$

for Region 3 and

$$
\omega_{1}^{\prime}=\frac{\omega \omega_{3}}{\omega_{2}}, \quad \omega_{3}^{\prime}=\frac{\omega \omega_{1}}{\omega_{2}}, \quad \omega_{2}^{\prime}=\frac{\omega^{2}}{\omega_{2}} .
$$

for Region 4.

\section{Appendix B. Angular integration in Eq. (2.1)}

In this appendix, we perform the angular integrations in Eq. (2.1) that yield Eq. (2.11). We have

$$
\begin{aligned}
U\left(\omega, \omega_{1}, \omega_{2}, \omega_{3}\right) & \equiv \frac{g^{4}}{32} \omega \omega_{1} \omega_{2} \omega_{3} \\
& \times \int \frac{\mathrm{d} \Omega_{1}}{(2 \pi)^{3}} \int \frac{\mathrm{d} \Omega_{2}}{(2 \pi)^{3}} \int \frac{\mathrm{d} \Omega_{3}}{(2 \pi)^{3}}(2 \pi)^{3} \delta\left(\boldsymbol{k}+\boldsymbol{k}_{1}-\boldsymbol{k}_{2}-\boldsymbol{k}_{3}\right) .
\end{aligned}
$$

Recall that $U$ shares the same symmetries as the matrix element as shown in Eq. (2.12) and that it is a homogeneous function of gluon frequencies whose degree of homogeneity is $\nu=-1$ (cf. Eq. (A.9)). Using the following integral representation of the delta distribution

$$
(2 \pi)^{3} \delta\left(\boldsymbol{k}+\boldsymbol{k}_{1}-\boldsymbol{k}_{2}-\boldsymbol{k}_{3}\right)=\mathrm{e}^{i \boldsymbol{x} \cdot\left(\boldsymbol{k}+\boldsymbol{k}_{1}-\boldsymbol{k}_{2}-\boldsymbol{k}_{3}\right)},
$$

one can integration over the solid angles separately,

$$
\int \frac{\mathrm{d} \Omega_{1}}{(2 \pi)^{3}} \mathrm{e}^{i \boldsymbol{x} \cdot \boldsymbol{k}_{1}}=\frac{1}{4 \pi^{2}} \int_{-1}^{1} \mathrm{~d} \cos \theta \mathrm{e}^{i|\boldsymbol{x}| \omega_{1} \cos \theta}=\frac{1}{2 \pi^{2}} \frac{1}{|\boldsymbol{x}| \omega_{1}} \sin \left(|\boldsymbol{x}| \omega_{1}\right),
$$

and one obtains

$$
\begin{aligned}
& U\left(\omega, \omega_{1}, \omega_{2}, \omega_{3}\right) \\
& =\frac{\lambda^{2}}{2(2 \pi)^{4}} \frac{1}{\omega^{2}} \int_{0}^{\infty} \frac{\mathrm{d}|\boldsymbol{x}|}{|\boldsymbol{x}|^{2}} \sin (|\boldsymbol{x}| \omega) \sin \left(|\boldsymbol{x}| \omega_{1}\right) \sin \left(|\boldsymbol{x}| \omega_{2}\right) \sin \left(|\boldsymbol{x}| \omega_{2}\right) .
\end{aligned}
$$


Then using the following result

$$
\int_{0}^{\infty} \frac{\mathrm{d}|\boldsymbol{x}|}{|\boldsymbol{x}|^{2}} \sin (|\boldsymbol{x}| \omega) \sin \left(|\boldsymbol{x}| \omega_{1}\right) \sin \left(|\boldsymbol{x}| \omega_{2}\right) \sin \left(|\boldsymbol{x}| \omega_{3}\right)=\frac{\pi}{4} \min \left(\omega, \omega_{1}, \omega_{2}, \omega_{3}\right)
$$

we readily find

$$
U\left(\omega, \omega_{1}, \omega_{2}, \omega_{3}\right)=\frac{g^{4}}{2(4 \pi)^{3}} \min \left(\omega, \omega_{1}, \omega_{2}, \omega_{3}\right)
$$

\section{Appendix C. Boltzmann-Nordheim equation in the small angle approximation}

Due to the Coulomb singularity, the 2 to 2 gluon scattering matrix element (3.1) diverges when $t \rightarrow 0$ (and $s \simeq-u$ ) in the third term and $u \rightarrow 0$ (and $s \simeq t$ ) in the fourth. In this limit, we have

$$
\left|M_{23 \rightarrow 1 k}\right|^{2} \equiv 8 N_{c}^{2} g^{4}\left(-\frac{u s}{t^{2}}-\frac{s t}{u^{2}}\right) \simeq 16 N_{c}^{2} g^{4} \frac{s^{2}}{t^{2}} \equiv 16 N_{c}^{2} g^{4} \frac{\left(k-k_{1}\right)^{4}}{\left(k-k_{2}\right)^{4}} .
$$

Introducing the momentum transfer $\boldsymbol{q}=\boldsymbol{k}-\boldsymbol{k}_{2}$ and by using the conservation of momenta, we can rewrite the incoming momenta $\boldsymbol{k}_{2}$ and $\boldsymbol{k}_{3}$ as a function of the outgoing momenta $\boldsymbol{k}$ and $\boldsymbol{k}_{1}$, and the new variable $\boldsymbol{q}$,

$$
\boldsymbol{k}_{2}=\boldsymbol{k}+\boldsymbol{q}, \quad \boldsymbol{k}_{3}=\boldsymbol{k}_{1}-\boldsymbol{q},
$$

Now, integrating over $\boldsymbol{k}_{2}$, the collision integral (2.1) reads

$$
I[n] \equiv \int_{1 \boldsymbol{q}} w(\boldsymbol{k} 1 \boldsymbol{q}) F[n]
$$

with $F[n]$ given by Eq. (2.2) and

$$
\left.w\left(\boldsymbol{k} \boldsymbol{k}_{1} \boldsymbol{q}\right) \equiv W\left(k k_{1} k_{2} k_{3}\right)(2 \pi) \delta\left(\omega+\omega_{1}-\omega_{2}-\omega_{3}\right)\right|_{\boldsymbol{k}_{3}=\boldsymbol{k}+\boldsymbol{k}_{1}-\boldsymbol{k}_{2}},
$$

where

$$
W\left(k k_{1} k_{2} k_{3}\right)=\frac{1}{32 \omega \omega_{1} \omega_{2} \omega_{3}}\left|M_{23 \rightarrow 1 k}\right|^{2}
$$


which is symmetric under any permutation of the four momenta. This symmetry translates in $w\left(\boldsymbol{k} \boldsymbol{k}_{1} \boldsymbol{q}\right)$ by

$$
w\left(\boldsymbol{k}_{1} \boldsymbol{q}\right)=w\left(\boldsymbol{k}_{1} \boldsymbol{k q}\right)=w\left(\boldsymbol{k}_{1} \boldsymbol{k},-\boldsymbol{q}\right)=w\left(\boldsymbol{k}_{1}-\boldsymbol{q}, \boldsymbol{k}+\boldsymbol{q}, \boldsymbol{q}\right) .
$$

In the limit where $\boldsymbol{q} \ll \boldsymbol{k}_{i}$ one can perform a gradient expansion of the collision integral. To do so, it is useful to multiply the Eq. (C.3) by a test function $g_{\boldsymbol{k}}$ and integrate over $\boldsymbol{k}$,

$$
\begin{aligned}
\int_{\boldsymbol{k}} g_{\boldsymbol{k}} I[n] & \equiv \int_{\boldsymbol{k} \boldsymbol{k}_{1} \boldsymbol{q}} g_{\boldsymbol{k}} w\left(\boldsymbol{k} \boldsymbol{k}_{1} \boldsymbol{q}\right), F[n] \\
& \equiv \frac{1}{2} \int_{\boldsymbol{k} \boldsymbol{k}_{1} \boldsymbol{q}}\left(g_{\boldsymbol{k}}-g_{\boldsymbol{k}+\boldsymbol{q}}\right) w\left(\boldsymbol{k} \boldsymbol{k}_{1} \boldsymbol{q}\right)\left(n_{\boldsymbol{k}_{1}}+1\right)\left(n_{\boldsymbol{k}}+1\right) n_{\boldsymbol{k}+\boldsymbol{q}} n_{\boldsymbol{k}_{1}-\boldsymbol{q}} \\
& +\frac{1}{2} \int_{\boldsymbol{k} \boldsymbol{k}_{1} \boldsymbol{q}}\left(g_{\boldsymbol{k}-\boldsymbol{q}}-g_{\boldsymbol{k}}\right) w\left(\boldsymbol{k}_{1} \boldsymbol{q}\right) n_{\boldsymbol{k}_{1}} n_{\boldsymbol{k}}\left(n_{\boldsymbol{k}-\boldsymbol{q}}+1\right)\left(n_{\boldsymbol{k}_{1}+\boldsymbol{q}}+1\right),
\end{aligned}
$$

where we have made an extensive use of Eq. (C.6). The first non-vanishing term in the expansion in powers of $\boldsymbol{q}$ is quadratic: the zeroth order vanishes identically, and owing to the fact that

$$
\int_{\boldsymbol{q}} q^{i} w\left(\boldsymbol{k} \boldsymbol{k}_{1} \boldsymbol{q}\right)=0,
$$

the linear term vanishes too. Thus, we obtain

$$
\begin{aligned}
& \int_{\boldsymbol{k}} g_{\boldsymbol{k}} I[n] \simeq \frac{1}{2} \int_{\boldsymbol{k} \boldsymbol{k}_{1} \boldsymbol{q}} q^{i} q^{j}\left(\nabla_{\boldsymbol{k}}^{i} g_{\boldsymbol{k}}\right) w\left(\boldsymbol{k} \boldsymbol{k}_{1} \boldsymbol{q}\right)\left[\left(n_{\boldsymbol{k}_{1}}+1\right)\left(n_{\boldsymbol{k}}+1\right)\right. \\
& \left.\times\left(n_{\boldsymbol{k}} \nabla_{1}^{i} n_{\boldsymbol{k}_{1}}-n_{\boldsymbol{k}_{1}} \nabla_{\boldsymbol{k}}^{i} n_{\boldsymbol{k}}\right)+n_{\boldsymbol{k}_{1}} n_{\boldsymbol{k}}\left(\left(n_{\boldsymbol{k}_{1}}+1\right) \nabla_{\boldsymbol{k}}^{i} n_{\boldsymbol{k}}-\left(n_{\boldsymbol{k}}+1\right) \nabla_{1}^{i} n_{\boldsymbol{k}_{1}}\right)\right] \\
& =\frac{1}{2} \int_{\boldsymbol{k} \boldsymbol{k}_{1} \boldsymbol{q}} q^{i} q^{j}\left(\nabla_{\boldsymbol{k}}^{i} g_{\boldsymbol{k}}\right) w\left(\boldsymbol{k}_{1} \boldsymbol{q}\right)\left[n_{\boldsymbol{k}}\left(n_{\boldsymbol{k}}+1\right) \nabla_{1}^{i} n_{\boldsymbol{k}_{1}}-n_{\boldsymbol{k}_{1}}\left(n_{\boldsymbol{k}_{1}}+1\right) \nabla_{\boldsymbol{k}}^{i} n_{\boldsymbol{k}}\right] \\
& =\frac{1}{2} \int_{\boldsymbol{k}} g_{\boldsymbol{k}} \nabla_{\boldsymbol{k}}^{i} \int_{\boldsymbol{k}_{1} \boldsymbol{q}} q^{i} q^{j} w\left(\boldsymbol{k}_{\boldsymbol{k}_{1}} \boldsymbol{q}\right)\left[n_{\boldsymbol{k}}\left(n_{\boldsymbol{k}}+1\right) \nabla_{1}^{i} n_{\boldsymbol{k}_{1}}-n_{\boldsymbol{k}_{1}}\left(n_{\boldsymbol{k}_{1}}+1\right) \nabla_{\boldsymbol{k}}^{i} n_{\boldsymbol{k}}\right]
\end{aligned}
$$

In the last step we have performed an integration by part where the surface term at infinity is assumed to vanish. This allows us, since the test function 
is arbitrary, to identify the integrant in the l.h.s to the one in the r.h.s., and as a result we obtain the Landau equation

$$
I[n] \equiv \nabla_{\boldsymbol{k}}^{i} \int_{\boldsymbol{k}_{1}} B^{i j}\left(\boldsymbol{k}_{\boldsymbol{k}_{1}}\right)\left[n_{\boldsymbol{k}_{1}}\left(n_{\boldsymbol{k}_{1}}+1\right) \nabla_{\boldsymbol{k}}^{i} n_{\boldsymbol{k}}-n_{\boldsymbol{k}}\left(n_{\boldsymbol{k}}+1\right) \nabla_{1}^{i} n_{\boldsymbol{k}_{1}}\right]
$$

where

$$
B^{i j}\left(\boldsymbol{k}_{1}\right)=\frac{1}{2} \int_{\boldsymbol{q}} q^{i} q^{j} w\left(\boldsymbol{k}_{1} \boldsymbol{q}\right)
$$

In a relativistic on-shell particle of momentum $k$, we have $k^{2}=0$, which yields the dispersion relation $\omega(\boldsymbol{k})=|\boldsymbol{k}|$. If follows that

$$
\left(k+k_{1}\right)^{4}=\left(2 k_{1} \cdot k\right)^{2}=4 \omega^{2} \omega_{1}^{2}\left(1-\boldsymbol{v} \cdot \boldsymbol{v}_{1}\right)^{2},
$$

where $\boldsymbol{v} \equiv \boldsymbol{k} /|\boldsymbol{k}|$ and $\boldsymbol{v}_{1} \equiv \boldsymbol{k}_{1} /\left|\boldsymbol{k}_{1}\right|$ stand for the velocity vectors. Similarly, using $\omega_{2}=\left|\boldsymbol{k}_{2}\right| \simeq|\boldsymbol{k}|+\boldsymbol{q} \cdot \boldsymbol{v}$ when $\boldsymbol{q} \ll \boldsymbol{k}$, we have

$$
\left(k-k_{2}\right)^{4}=\left[\left(\omega-\omega_{2}\right)^{2}-\boldsymbol{q}^{2}\right]^{2} \simeq\left[(\boldsymbol{v} \cdot \boldsymbol{q})^{2}-\boldsymbol{q}^{2}\right]^{2} .
$$

Finally, in this approximation the argument of the delta function in Eq. (C.4) reads

$$
\omega(\boldsymbol{k})+\omega\left(\boldsymbol{k}_{1}\right)-\omega\left(\boldsymbol{k}_{2}\right)-\omega\left(\boldsymbol{k}_{3}\right) \simeq \boldsymbol{v} \cdot \boldsymbol{q}-\boldsymbol{v}_{1} \cdot \boldsymbol{q} .
$$

As a result, we find in the small angle approximation, i.e. $\boldsymbol{q} \ll \boldsymbol{k}, \boldsymbol{k}_{1}$,

$$
w\left(\boldsymbol{k} \boldsymbol{k}_{1} \boldsymbol{q}\right) \simeq 4 \pi N_{c}^{2} g^{4} \frac{\left(1-\boldsymbol{v} \cdot \boldsymbol{v}_{1}\right)^{2}}{\left[(\boldsymbol{v} \cdot \boldsymbol{q})^{2}-\boldsymbol{q}^{2}\right]^{2}} \delta\left(\boldsymbol{v} \cdot \boldsymbol{q}-\boldsymbol{v}_{1} \cdot \boldsymbol{q}\right)
$$

Hence, (dropping for the moment the constant factor $4 \pi N_{c}^{2} g^{4}$ )

$$
B^{i j}\left(\boldsymbol{k}_{1}\right)=\frac{1}{2} \int_{\boldsymbol{q}} q^{i} q^{j} \frac{\left(1-\boldsymbol{v} \cdot \boldsymbol{v}_{1}\right)^{2}}{\left[(\boldsymbol{v} \cdot \boldsymbol{q})^{2}-\boldsymbol{q}^{2}\right]^{2}} \delta\left(\boldsymbol{v} \cdot \boldsymbol{q}-\boldsymbol{v}_{1} \cdot \boldsymbol{q}\right) .
$$

Now, it is useful to make the following change of variables:

$$
\boldsymbol{w} \equiv \boldsymbol{v}-\boldsymbol{v}_{1} \quad \text { and } \quad \boldsymbol{u} \equiv \boldsymbol{v}+\boldsymbol{v}_{1}
$$

Using the fact that $\boldsymbol{v}^{2}=\boldsymbol{v}_{1}^{2}=0$ and that the vectors $\boldsymbol{u}$ and $\boldsymbol{w}$ are orthogonal $\boldsymbol{w} \cdot \boldsymbol{u}=0 \mathrm{Eq}$. C.16 can be decomposed as follows

$$
B^{i j}\left(\boldsymbol{k}_{1}\right)=B_{1}\left(\delta^{i j}-\frac{w^{i} w^{j}}{\boldsymbol{w}^{2}}\right)+B_{2} \frac{u^{i} u^{j}}{\boldsymbol{u}^{2}}
$$


where

$$
\boldsymbol{u} \cdot \boldsymbol{B}_{1} \boldsymbol{u}=\left(B_{1}+B_{2}\right) \boldsymbol{u}^{2}, \quad \text { and } \quad \operatorname{Tr} \boldsymbol{B}=2 B_{1}+B_{2} .
$$

Inverting Eq. (C.19) yields

$$
B_{1}=\operatorname{Tr} \boldsymbol{B}-\frac{\boldsymbol{u} \cdot \boldsymbol{B} \boldsymbol{u}}{\boldsymbol{u}^{2}}, \quad \text { and } \quad B_{2}=-\operatorname{Tr} \boldsymbol{B}+2 \frac{\boldsymbol{u} \cdot \boldsymbol{B} \cdot \boldsymbol{u}}{\boldsymbol{u}^{2}} .
$$

Note also the following identities

$$
1-\boldsymbol{v} \cdot \boldsymbol{v}_{1}=\frac{1}{2} \boldsymbol{w}^{2}, \quad 1+\boldsymbol{v} \cdot \boldsymbol{v}_{1}=\frac{1}{2} \boldsymbol{u}^{2}, \quad \text { and } \quad \boldsymbol{w}^{2}+\boldsymbol{u}^{2}=4
$$

Thereby, we have

$$
\operatorname{Tr} \boldsymbol{B} \equiv \int_{\boldsymbol{q}} \frac{\boldsymbol{q}^{2} \boldsymbol{w}^{2}}{\left[(\boldsymbol{u} \cdot \boldsymbol{q})^{2}-4 \boldsymbol{q}^{2}\right]^{2}} \delta(\boldsymbol{w} \cdot \boldsymbol{q})
$$

and

$$
\frac{\boldsymbol{u} \cdot \boldsymbol{B} \cdot \boldsymbol{u}}{\boldsymbol{u}^{2}} . \equiv \int_{\boldsymbol{q}} \frac{(\boldsymbol{u} \cdot \boldsymbol{q})^{2}}{\boldsymbol{u}^{2}\left[(\boldsymbol{u} \cdot \boldsymbol{q})^{2}-4 \boldsymbol{q}^{2}\right]^{2}} \delta(\boldsymbol{w} \cdot \boldsymbol{q})
$$

In order to perform the $\boldsymbol{q}$ integration we chose the reference frame where $\boldsymbol{w}$ is along the $z$ axis and thus $\boldsymbol{u}$ is in the $x y$ plan. In spherical coordinates, we have $\boldsymbol{w} \cdot \boldsymbol{q}=|\boldsymbol{w}||\boldsymbol{q}| \cos \theta$ and $\boldsymbol{u} \cdot \boldsymbol{q}=|\boldsymbol{w} \| \boldsymbol{q}| \sin \theta \sin \phi$. Hence,

$$
\begin{aligned}
B_{1} & =\frac{2}{(2 \pi)^{3}} \int \mathrm{d}|\boldsymbol{q} \| \boldsymbol{q}|^{2} \int_{0}^{2 \pi} \mathrm{d} \phi \int_{0}^{2 \pi} \mathrm{d} \cos \phi \\
& \times \frac{(\boldsymbol{u} \cdot \boldsymbol{q})^{2}}{\boldsymbol{u}^{2}\left[(\boldsymbol{u} \cdot \boldsymbol{q})^{2}-4 \boldsymbol{q}^{2}\right]^{2}} \delta(|\boldsymbol{w}||\boldsymbol{q}| \cos \phi) \\
& =\frac{2|\boldsymbol{w}|^{3}}{(2 \pi)^{3}} \int \frac{\mathrm{d}|\boldsymbol{q}|}{|\boldsymbol{q}|} \int_{0}^{2 \pi} \mathrm{d} \phi \frac{1-\sin ^{2} \phi}{\boldsymbol{u}^{2} \sin ^{2} \phi-4} .
\end{aligned}
$$

The integration over $\boldsymbol{q}$ is logarithmically divergent and yields the Coulomb $\operatorname{logarithm} \lambda$. The remaining integral over $\phi$ yields

$$
\int_{0}^{2 \pi} \mathrm{d} \phi \frac{1-\sin ^{2} \phi}{\boldsymbol{u}^{2} \sin ^{2} \phi-4}=\frac{\pi}{8 \sqrt{4-\boldsymbol{u}^{2}}}=\frac{\pi}{8|\boldsymbol{w}|} .
$$


We finally obtain,

$$
B_{1}=\frac{\lambda}{8(2 \pi)^{2}}|\boldsymbol{w}|^{2}, \quad B_{2}=\frac{\lambda}{8(2 \pi)^{2}},
$$

where, for $B_{2}$, we have used

$$
\int_{0}^{2 \pi} \mathrm{d} \phi \frac{2 \sin ^{2} \phi-1}{\boldsymbol{u}^{2} \sin ^{2} \phi-4}=\frac{\pi \boldsymbol{u}^{2}}{8\left(4-\boldsymbol{u}^{2}\right)^{3 / 2}}=\frac{\pi \boldsymbol{u}^{2}}{8|\boldsymbol{w}|^{3 / 2}} .
$$

Finally, $B^{i j}$ reads

$$
B^{i j}=\frac{\lambda}{8(2 \pi)^{2}}\left[\left(\delta^{i j}-\frac{w^{i} w^{j}}{|\boldsymbol{w}|^{2}}\right)|\boldsymbol{w}|^{2}+u^{i} u^{j}\right] .
$$

which implies that $w^{i} B^{i j}=0$. For an isotropic medium, the Fokker-Planck equation simplifies further. We first rewrite the collision term as

$$
I[n] \equiv \nabla_{\boldsymbol{k}}^{i}\left[D_{\boldsymbol{k}}^{i j} \nabla_{\boldsymbol{k}}^{j} n_{\boldsymbol{k}}-C_{\boldsymbol{k}}^{i} n_{\boldsymbol{k}}\left(n_{\boldsymbol{k}}+1\right)\right]
$$

with

$$
D_{\boldsymbol{k}}^{i j} \equiv \int_{1} B^{i j}\left(\boldsymbol{k}_{1}\right) n_{\boldsymbol{k}_{1}}\left(n_{\boldsymbol{k}_{1}}+1\right), \quad \text { and } \quad C_{\boldsymbol{k}}^{i} \equiv \int_{1} B^{i j}\left(\boldsymbol{k} \boldsymbol{k}_{1}\right) \nabla_{1}^{j} n_{\boldsymbol{k}_{1}}
$$

Now, in the isotropic case, $C_{\boldsymbol{k}}^{i} \propto v^{i}$ and using the fact that $w^{i} B^{i j}=0$, we find

$$
C_{\boldsymbol{k}}^{i} \equiv v^{i} \int_{1} \boldsymbol{v} \cdot \boldsymbol{B}\left(\boldsymbol{k} \boldsymbol{k}_{1}\right) \cdot \nabla_{1} n_{\boldsymbol{k}_{1}}=\frac{1}{2} v^{i} \int_{1} \boldsymbol{u} \cdot \boldsymbol{B}\left(\boldsymbol{k} \boldsymbol{k}_{1}\right) \cdot \nabla_{1} n_{\boldsymbol{k}_{1}}
$$

Using Eq. (C.28) and recalling that $\boldsymbol{u}=\boldsymbol{v}_{1}-\boldsymbol{v}$, the term proportional to $\boldsymbol{v}$ is a total divergence and therefore vanishes assuming that $n_{\boldsymbol{k}}$ vanishes at $\boldsymbol{k} \rightarrow \infty$. Hence, integrating by part using $\boldsymbol{\nabla}_{1} \boldsymbol{v}_{1}=1 /\left|\boldsymbol{k}_{1}\right|$, we obtain

$$
C_{\boldsymbol{k}}^{i}=\frac{\lambda}{8(2 \pi)^{2}} v^{i} \int_{1} \frac{n_{\boldsymbol{k}_{1}}}{\left|\boldsymbol{k}_{1}\right|} \equiv \frac{\lambda}{16(2 \pi)^{2}} v^{i} I_{b}
$$

where $I_{b}$ is given in Eq. (3.5). Let us turn now to $D^{i j}$. It takes to form $D^{i j}=D_{1} \delta^{i j}+D_{2} v^{i} v^{j}$ where,

$$
D_{1} \equiv \frac{1}{2}(\operatorname{Tr} \boldsymbol{D}-\boldsymbol{v} \cdot \boldsymbol{D} \cdot \boldsymbol{v}) \quad \text { and } \quad D_{2} \equiv-\frac{1}{3} \operatorname{Tr} \boldsymbol{D}+\boldsymbol{v} \cdot \boldsymbol{D} \cdot \boldsymbol{v}
$$


From Eq. (C.28) and using Eq. (C.21), it follows that

$$
\begin{aligned}
\operatorname{Tr} \boldsymbol{D} & =\frac{\lambda}{8(2 \pi)^{2}} \int_{1}\left(6+2 \boldsymbol{v}_{1} \cdot \boldsymbol{v}\right) n_{1}\left(n_{1}+1\right) \\
& =\frac{\lambda}{8(2 \pi)^{2}} 6 \int_{1} n_{1}\left(n_{1}+1\right) \\
& =\frac{\lambda}{8(2 \pi)^{2}} 6 I_{a},
\end{aligned}
$$

where the second term in the r.h.s. of the first vanishes due to the symmetry under the parity transformation $\boldsymbol{v}_{1} \rightarrow-\boldsymbol{v}_{1}$. The integral $I_{a}$ is introduced in Eq. (3.5). Similarly, we obtain

$$
\boldsymbol{v} \cdot \boldsymbol{D} \cdot \boldsymbol{v}=\frac{\lambda}{8(2 \pi)^{2}} 2 I_{a} .
$$

Now, using Eqs. (C.34) and (C.35) in (C.33), we find

$$
D_{1} \equiv \frac{\lambda}{4(2 \pi)^{2}} I_{a} \quad \text { and } \quad D_{2}=0,
$$

or

$$
D_{\boldsymbol{k}}^{i j} \equiv \frac{\lambda}{4(2 \pi)^{2}} I_{a} \delta^{i j} .
$$

The Fokker-Planck equation can be then rewritten in the form (putting back the prefactor $\left.4 \pi N_{c}^{2} g^{4}\right)$

$$
I[n] \equiv 4 \pi N_{c}^{2} g^{4} \frac{\lambda}{16(2 \pi)^{2}} \boldsymbol{\nabla}_{\boldsymbol{k}} \cdot\left[I_{a} \boldsymbol{\nabla}_{\boldsymbol{k}} n_{\boldsymbol{k}}+I_{b} \frac{\boldsymbol{k}}{|\boldsymbol{k}|} n_{\boldsymbol{k}}\left(n_{\boldsymbol{k}}+1\right)\right]
$$

\section{Appendix D. Steady state solution of the Fokker-Planck equation}

In this appendix we will derive the stationary solution to the FokkerPlanck equation (3.13), in the presence of a steady source that inject particles at a forcing scale $\omega_{f}$ with a constant rate $Q_{f}$. We recall that

$$
Q \equiv \frac{1}{4} \hat{q} \omega^{2}\left[\frac{\partial}{\partial \omega} n(\omega)+\frac{n^{2}(\omega)}{T_{*}}\right]+Q_{f} \theta\left(\omega-\omega_{f}\right)=\text { const. }
$$


Eq. (D.1) is a first order non-linear differential equation where $Q$ is a constant to be fixed by boundary conditions. It is a Ricatti type equation that can be reduced to a first order linear ordinary differential equation. Let us look for a solution of the form

$$
n(\omega)=T_{*}\left[\frac{C}{\omega}+\frac{1}{y(\omega)}\right]
$$

$C$ being a constant. In terms of the new function $y(\omega)$, Eq. (3.16) reads

$$
-\frac{C}{\omega^{2}}-\frac{y^{\prime}}{y^{2}}+\frac{C^{2}}{\omega^{2}}+\frac{2 C}{\omega y}+\frac{1}{\omega^{2}}-\frac{D}{\omega^{2}}=0,
$$

with

$$
D \equiv D(\omega)=\frac{4\left(Q-Q_{f}\right)}{\hat{q} T_{*}} \theta\left(\omega-\omega_{f}\right)
$$

Now, $C$ can be chosen to cancel the terms that are proportional to $\omega^{-2}$. As a result, we are left with a quadratic equation to solve for $C$,

$$
C^{2}-C-D=0
$$

and a first order differential equation for $y$,

$$
-y^{\prime}+\frac{2 C}{\omega} y+1=0 .
$$

Eq. (D.5) admits two solutions,

$$
C_{ \pm}=\frac{1}{2}(1 \pm \sqrt{1+4 D}), \quad \text { for } \quad D>-\frac{1}{4},
$$

and Eq. (D.6) admits the particular solution

$$
y_{p}=\frac{\omega}{1-2 C}=\mp \frac{\omega}{\sqrt{1+4 D}}
$$

which is to be added to the general solution of the homogenous equation

$$
-y^{\prime}+\frac{2 C}{\omega} y=0
$$


that is

$$
y_{h}=A \omega^{2 C} .
$$

Finally the general solution of Eq. (D.1), that is symmetric in the exchange of $C_{+}$and $C_{-}$, reads

$$
n_{\mathrm{st}}(\omega)=\frac{T_{*}}{2 \omega}\left(1+\beta+\frac{2 \beta}{A k^{\beta}-1}\right),
$$

where $\beta(\omega)=\sqrt{1+4 D(\omega)}$.

Let us now specify the boundary conditions. We require the presence of sinks to remove particles below the infrared scale $\omega_{-} \ll \omega_{f}$ and above the UV scale $\omega_{+} \gg \omega_{f}$. The sinks are accounted for by imposing the following Dirichlet boundary conditions:

$$
n\left(\omega_{-}\right)=n\left(\omega_{+}\right)=0 .
$$

These boundary conditions (D.12) applied to the general solution (D.11),

$$
1+\beta+\frac{2 \beta}{A \omega_{-}^{\beta}-1}=0,
$$

to the left of the forcing scale, allow us to determine the constant $C$,

$$
A=\frac{1-\beta}{1+\beta} \omega_{-}^{-\beta} \text {. }
$$

Thereby, for $\omega<\omega_{f}$, the steady state solution reads

$$
n_{\mathrm{st}}(\omega)=\frac{T_{*}(1+\beta)}{2 \omega}\left(1+\frac{2 \beta}{(1-\beta)\left(\omega / \omega_{-}\right)^{\beta}-1-\beta}\right),
$$

with $\beta=\sqrt{1+\frac{16 Q}{\hat{q} T_{*}}}$, and similarly, to right of the forcing scale, $\omega>\omega_{f}$, we obtain

$$
n_{\mathrm{st}}(\omega)=\frac{T_{*}(1+\beta)}{2 \omega}\left(1+\frac{2 \beta}{(1-\beta)\left(\omega / \omega_{+}\right)^{\beta}-1-\beta}\right),
$$

with $\beta=\sqrt{1+\frac{16\left(Q-Q_{f}\right)}{\hat{q} T_{*}}}$. It is instructive to analyze the solution away from the sinks. For $\omega_{-} \ll \omega<\omega_{f}$, we have

$$
n_{\mathrm{st}}(\omega) \simeq\left(1+\sqrt{1+\frac{16 Q}{\hat{q} T_{*}}}\right) \frac{T_{*}}{2 \omega},
$$


and for $\omega_{f}<\omega \ll \omega_{+}$, we have

$$
n_{\mathrm{st}}(\omega) \simeq\left(1-\sqrt{1+\frac{16\left(Q-Q_{f}\right)}{\hat{q} T_{*}}}\right) \frac{T_{*}}{2 \omega},
$$

We note a discontinuity of the solution at the forcing scale which is confirmed by explicit numerical solutions. In the case where the sinks and sources are well separated we expect $Q \simeq Q_{f}$, which implies that $n_{\text {st }}(\omega)=0$ to the right of the forcing scale.

\section{Appendix E. Collinear divergence in 2 to 3 processes}

In this appendix we analyze the next to leading order contribution to the collision integral that corresponds to inelastic 2 to 3 (and 3 to 2) processes depicted in Figure. E.17 [42]. In particular, we show that the corresponding matrix element is singular in the collinear limit and see how this singularity is cured, in the Hard-Loop approximation, by accounting for collective effects in the gluon self energy (See also Ref. [43] for a similar discussion).

Consider the 2 to 3 scattering process, $k_{1}+k_{2} \rightarrow k_{3}+k_{4}+k$, where $k$ and $k_{i}$, with $(i=1,2,3,4)$, stand for the measured gluon in the final state and the remaining unmeasured ones, respectively. The collision integral reads

$$
\begin{aligned}
I_{2 \rightarrow 3}[n] & \equiv \frac{1}{2 ! 2 !(2 \omega)} \int_{1,2,3,4}\left|M_{12 \rightarrow 34 k}\right|^{2} \\
& \times F_{2 \rightarrow 3}[n](2 \pi)^{4} \delta^{(4)}\left(k_{1}+k_{2}-k_{3}-k_{4}-k\right),
\end{aligned}
$$

where

$$
F_{2 \rightarrow 3}[n] \equiv n_{1} n_{2}\left(n_{3}+1\right)\left(n_{4}+1\right)\left(n_{k}+1\right)-\left(n_{1}+1\right)\left(n_{2}+1\right) n_{3} n_{4} n_{k},
$$

Similarly, for the 3 to 2 process, $k_{1}+k_{2}+k_{3} \rightarrow k_{4}+k$, we have

$$
\begin{aligned}
I_{3 \rightarrow 2}[n] & \equiv \frac{1}{3 !(2 \omega)} \int_{1,2,3,4}\left|M_{123 \rightarrow 4 k}\right|^{2} \\
& \times F_{3 \rightarrow 2}[n](2 \pi)^{4} \delta^{(4)}\left(k_{1}+k_{2}+k_{3}-k_{4}-k\right),
\end{aligned}
$$

where

$$
F_{3 \rightarrow 2}[n] \equiv n_{1} n_{2} n_{3}\left(n_{4}+1\right)\left(n_{k}+1\right)-\left(n_{1}+1\right)\left(n_{2}+1\right)\left(n_{3}+1\right) n_{4} n_{k} .
$$


The factors $1 / 2 ! 2$ ! and $1 / 3$ ! are symmetry factors.

Here, the invariant matrix element squared is given by [42]

$$
\begin{aligned}
& \left|M_{12 \rightarrow 345}\right|^{2}=\frac{1}{2} g^{6} \frac{N_{c}^{3}}{N_{c}^{2}-1} \frac{N}{D}[(12345)+(1254)+(12435)+(12453) \\
& +(12534)+(12543)+(13245)+(13254)+(13425)+(13524) \\
& +(14235)+(14325)]
\end{aligned}
$$

with

$$
\begin{aligned}
N & =(12)^{4}+(13)^{4}+(14)^{4}+(15)^{4}+(23)^{4} \\
& +(24)^{4}+(25)^{4}+(34)^{4}+(35)^{4}+(45)^{4}
\end{aligned}
$$

and

$$
D=(12)(13)(14)(15)(23)(24)(25)(34)(35)(45)
$$

where $(i j) \equiv k_{i} \cdot k_{j}$ and $(i j k l m) \equiv(i j)(j k)(k l)(l m)(m j)$.

If the perturbation expansion were well behaved, this contribution should be sub-leading. However, as we shall see in this appendix, the 2 to 3 matrix element possesses a collinear divergence that invalidate the naive perturbative expansion.

As alluded to above, this matrix element possesses a collinear divergence that invalidate the naive perturbative expansion. To see this, let us consider Eq. (E.5) with $k_{5} \equiv k$, in the limit where gluons 2 and 4 are collinear. The result will then be multiplied by $2 ! 2$ ! to account for the remaining symmetric contributions. In this case, we have $t^{\prime} \equiv q^{2}=\left(k_{4}-k_{2}\right)^{2}=-2 k_{2} \cdot k_{4} \rightarrow 0$ and as a consequence of momentum conservation, it follows that the outgoing gluons 3 and $k$ are collinear to gluon 1 , that is, $t \equiv\left(k_{3}-k_{1}\right)^{2}=-2 k_{1} \cdot k_{3} \rightarrow 0$ and $t \equiv\left(k-k_{1}\right)^{2}=-2 k_{1} \cdot k \approx \omega \omega_{1} \theta_{1 k}^{2} \rightarrow 0$, that effectively corresponds to a small angle scattering (or small momentum transfer $q$ ) of gluon 2 into 4 . This process is depicted in Figure E.18.

For reasons that will later be clear, it is convenient to choose a frame where $\boldsymbol{k}_{1}$ is along the $z$ axis and adopt the light-cone variables, i.e., $k_{1} \equiv$ $\left(k_{1}^{+}, 0^{-}, 0_{\perp}\right)$. Keeping only the leading contributions in the collinear limit, $q \rightarrow 0$, and defining the momentum fraction $z=k^{+} / k_{1}^{+} \approx \omega / \omega_{1}$, we find 


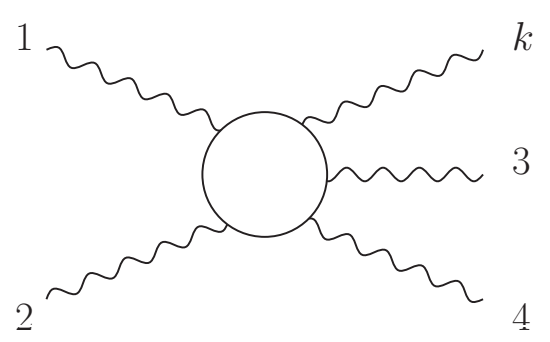

(a)

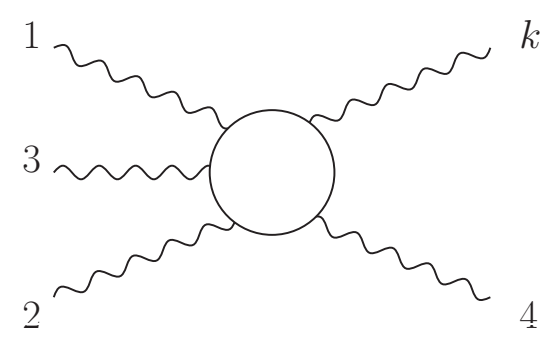

(b)

Figure E.17: Depiction of the 2 to 3 (a) and 3 to 2 (b) inelastic processes. Only the gain contributions to the collision integral are shown (with the gluon $k$ is measured in the final state).

that $2\left(k_{2} \cdot k_{4}\right) \equiv q^{2} \simeq-\boldsymbol{q}_{\perp}^{2}$ and

$$
\begin{array}{rlrl}
\left(k_{1} \cdot k_{2}\right) & \simeq\left(k_{1} \cdot k_{4}\right) \simeq \frac{s}{2}, & \left(k_{2} \cdot k\right) & \simeq\left(k_{4} \cdot k\right) \simeq \frac{s}{2} z, \\
\left(k_{2} \cdot k_{3}\right) & \simeq\left(k_{3} \cdot k_{4}\right) \simeq \frac{s}{2}(1-z), & \left(k_{1} \cdot k\right) \simeq \frac{\boldsymbol{k}_{\perp}^{2}}{2 z} \\
\left(k_{1} \cdot k_{3}\right) & \simeq \frac{(\boldsymbol{q}-\boldsymbol{k})_{\perp}^{2}}{2(1-z)}, & \left(k_{3} \cdot k\right) & \simeq \frac{(\boldsymbol{k}-z \boldsymbol{q})_{\perp}^{2}}{2 z(1-z)} .
\end{array}
$$

In the numerator $N$ (cf. Eq. (E.5)) yields

$$
\begin{aligned}
& (12)^{4}+(13)^{4}+(14)^{4}+(15)^{4}+(23)^{4}+(24)^{4}+(25)^{4} \\
& +(34)^{4}+(35)^{4}+(45)^{4} \\
& \simeq(12)^{4}+(14)^{4}+(15)^{4}+(23)^{4}+(25)^{4}+(34)^{4}+(45)^{4} \\
& \simeq \frac{s^{4}}{8}\left[1+z^{4}+(1-z)^{4}\right]=\frac{s^{4} z(1-z)}{8 N_{c}} P(z),
\end{aligned}
$$

Similarly, the sum of the terms $(i j k l m)$ in Eq. (E.5) reads

$$
\frac{s^{4}}{2^{4}}\left[(1-z)^{2} \boldsymbol{k}_{\perp}{ }^{2}+z^{2}(\boldsymbol{k}-\boldsymbol{q})_{\perp}^{2}+(\boldsymbol{k}-z \boldsymbol{q})_{\perp}^{2}\right],
$$

and the denominator $D$ yields

$$
\begin{aligned}
(12)(13)(14) & (15)(23)(24)(25)(34)(35)(45) \\
& \simeq \frac{s^{6}}{2^{10}} \boldsymbol{q}_{\perp}^{2} \boldsymbol{k}_{\perp}^{2}(\boldsymbol{k}-\boldsymbol{q})_{\perp}^{2}(\boldsymbol{k}-z \boldsymbol{q})_{\perp}^{2} .
\end{aligned}
$$




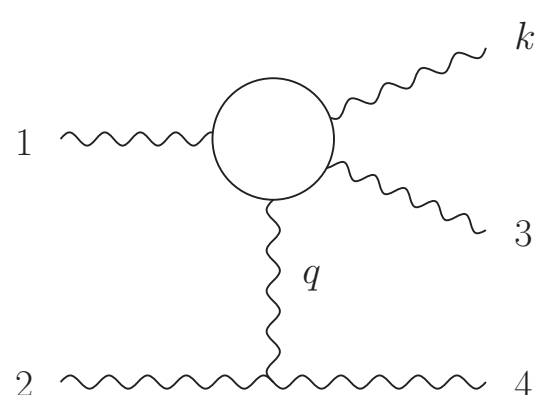

(a)

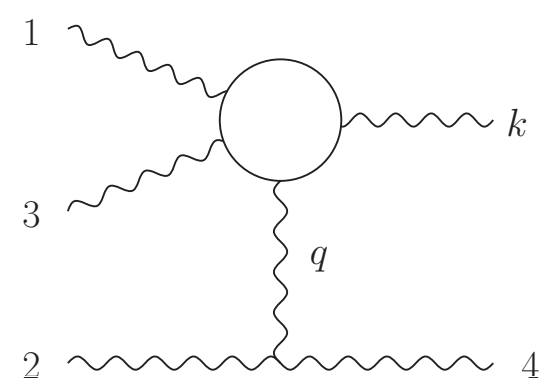

(b)

Figure E.18: Diagrammatic representation of the dominant contribution to the 2 to 3 (a) and 3 to 2 (b) processes shown in Figure E.17, that corresponds to the quasi-collinear 1 to 2 branching (merging) induced by a small momentum transfer $q$.

Inserting Eqs. (E.9), (E.10) and (E.11) in Eq. (E.5), we finally obtain

$$
\begin{aligned}
& \left|M_{12 \rightarrow 34 k}\right|^{2} \simeq \frac{4 g^{6} N_{c}^{2} s^{2}}{N_{c}^{2}-1} z(1-z) P(z) \\
& \times \frac{(1-z)^{2} \boldsymbol{k}_{\perp}{ }^{2}+z^{2}(\boldsymbol{k}-\boldsymbol{q})_{\perp}^{2}+(\boldsymbol{k}-z \boldsymbol{q})_{\perp}^{2}}{\boldsymbol{q}_{\perp}^{2} \boldsymbol{k}_{\perp}^{2}(\boldsymbol{k}-\boldsymbol{q})_{\perp}^{2}(\boldsymbol{k}-z \boldsymbol{q})_{\perp}^{2}} .
\end{aligned}
$$

Note that in the limit $\boldsymbol{q}_{\perp} \rightarrow 0$, the matrix element, Eq. (E.12), diverges as $\boldsymbol{q}^{-4}$. Likewise, when $\boldsymbol{k}_{\perp} \rightarrow 0$, the matrix element diverges as $\boldsymbol{k}^{-4}$. In the next section we shall see that this divergence is cut-off by screening effects, but for the time being, we assume that Eq. (E.12) is a well defined quantity to derive the general form the collision integral in the small angle approximation.

Now, the integration over $k_{3}$ (for instance) is trivial with the help of the 3-momentum delta function, $\boldsymbol{k}_{3}=\boldsymbol{k}_{1}-\boldsymbol{k}+\boldsymbol{q}$. In terms of the new variables, the integration measure transforms to

$$
\begin{aligned}
\frac{1}{4 \omega_{3} \omega} \int_{1,2,4} & \equiv \frac{1}{4 \omega_{3} \omega} \int \frac{\mathrm{d}^{3} k_{1}}{(2 \pi)^{3} 2 \omega_{1}} \int \frac{\mathrm{d}^{3} k_{2}}{(2 \pi)^{3} 2 \omega_{2}} \int \frac{\mathrm{d}^{3} k_{4}}{(2 \pi)^{3} 2 \omega_{4}} \\
& =\frac{1}{8(2 \pi)} \int \frac{\mathrm{d}^{3} k_{2}}{(2 \pi)^{3}} \int \frac{\mathrm{d} z}{z^{4}(1-z)} \int \frac{\mathrm{d}^{2} \boldsymbol{k}_{\perp}}{(2 \pi)^{2}} \int \frac{\mathrm{d} q_{z} \mathrm{~d}^{2} \boldsymbol{q}_{\perp}}{(2 \pi)^{3}} \frac{1}{\omega_{1}^{2} \omega_{2}^{2}}
\end{aligned}
$$


where $\omega_{1}=\omega / z$ and $\omega_{3}=\omega(1-z) / z$, and we have used that $\omega_{4} \simeq \omega_{2}$. The remaining (energy conserving) delta function yields

$$
\begin{aligned}
\delta\left(\omega_{1}+\omega_{2}\right. & \left.-\omega_{3}-\omega_{4}-\omega\right) \\
& =\delta\left(\left|\boldsymbol{k}_{1}\right|+\left|\boldsymbol{k}_{2}\right|-\left|\boldsymbol{k}_{1}-\boldsymbol{k}+\boldsymbol{q}\right|-\left|\boldsymbol{k}_{2}+\boldsymbol{q}\right|-|\boldsymbol{k}|\right) \\
& \simeq \delta\left(\left|\boldsymbol{k}_{1}\right|-\left|\boldsymbol{k}_{1}-\boldsymbol{k}\right|-|\boldsymbol{k}|-\frac{\boldsymbol{q} \cdot \boldsymbol{k}_{2}}{\left|\boldsymbol{k}_{2}\right|}-\frac{\boldsymbol{q} \cdot\left(\boldsymbol{k}_{1}-\boldsymbol{k}\right)}{\left|\boldsymbol{k}_{1}-\boldsymbol{k}\right|}\right),
\end{aligned}
$$

where, in terms of $q_{z}$ and $q_{\perp}$,

$$
\frac{\boldsymbol{q} \cdot \boldsymbol{k}_{2}}{\left|\boldsymbol{k}_{2}\right|}-\frac{\boldsymbol{q} \cdot\left(\boldsymbol{k}_{1}-\boldsymbol{k}\right)}{\left|\boldsymbol{k}_{1}-\boldsymbol{k}\right|} \simeq q_{z}\left(\frac{k_{2 z}}{\left|\boldsymbol{k}_{2}\right|}-1\right)-\frac{\boldsymbol{q}_{\perp} \cdot \boldsymbol{k}_{\perp 2}}{\left|\boldsymbol{k}_{2}\right|}-\frac{\boldsymbol{q}_{\perp} \cdot\left(\boldsymbol{k}_{1}-\boldsymbol{k}\right)_{\perp}}{\left|\boldsymbol{k}_{1}-\boldsymbol{k}\right|}
$$

This allows us to integrate over $q_{z}$ after turning the integration over $k_{4}$ into an integration over $q$ (cf. Eq. (E.13)). As $\left(\left|\boldsymbol{k}_{2}\right|-k_{2 z}\right) /\left|\boldsymbol{k}_{2}\right| \simeq 1-\cos \theta_{12}$, the integral over $q_{z}$ yields a factor $1 /\left(1-\cos \theta_{12}\right)$. Using $s=2 \omega_{1} \omega_{2}\left(1-\cos \theta_{21}\right)^{2}$ and assuming an isotropic medium the integration over $\theta_{12}$ yields

$$
\int_{-1}^{1} \mathrm{~d} \cos \theta_{12}\left(1-\cos \theta_{12}\right)=2 \text {. }
$$

Finally, one can express the collision integral in the small angle approximation as an effective quasi-collinear 1 to 2 process

$$
I_{2 \rightarrow 3}[n] \simeq I_{\text {"1 } \rightarrow 2 "}[n] \equiv \int \frac{\mathrm{d} z}{z^{3}} \mathcal{K}(z) F_{1 \rightarrow 2}[n],
$$

where

$$
\begin{aligned}
& F_{1 \rightarrow 2}[n] \equiv n_{1}\left(n_{k}+1\right)\left(n_{3}+1\right)-\left(n_{1}+1\right) n_{k} n_{k} \\
& =n\left(\frac{\omega}{z}\right)[n(\omega)+1][n((1-z) \omega / z)+1] \\
& -[n(\omega / z)+1] n(\omega) n((1-z) \omega / z),
\end{aligned}
$$

and

$$
\begin{aligned}
\mathcal{K}(z) \quad \equiv & \frac{4 \alpha_{s} g^{4} N_{c}^{2} I_{a}}{N_{c}^{2}-1} P(z) \int \frac{\mathrm{d}^{2} \boldsymbol{k}_{\perp}}{(2 \pi)^{2}} \int \frac{\mathrm{d}^{2} \boldsymbol{q}_{\perp}}{(2 \pi)^{2}} \\
& \times \frac{(1-z)^{2} \boldsymbol{k}_{\perp}^{2}+z^{2}(\boldsymbol{k}-\boldsymbol{q})_{\perp}^{2}+(\boldsymbol{k}-z \boldsymbol{q})_{\perp}^{2}}{\boldsymbol{q}_{\perp}^{2} \boldsymbol{k}_{\perp}^{2}(\boldsymbol{k}-\boldsymbol{q})_{\perp}^{2}(\boldsymbol{k}-z \boldsymbol{q})_{\perp}^{2}} . \\
& =\frac{4 \alpha_{s} g^{4} N_{c}^{2} I_{a}}{N_{c}^{2}-1} P(z) \int \frac{\mathrm{d}^{2} \boldsymbol{k}_{\perp}}{(2 \pi)^{2}} \int \frac{\mathrm{d}^{2} \boldsymbol{q}_{\perp}}{(2 \pi)^{2}} \frac{1+z^{2}+(1-z)^{2}}{\boldsymbol{q}_{\perp}^{2} \boldsymbol{k}_{\perp}^{2}(\boldsymbol{k}-\boldsymbol{q})_{\perp}^{2}} .
\end{aligned}
$$


with (cf. Eq. (3.5))

$$
I_{a} \equiv \int \frac{\mathrm{d}^{3} k_{2}}{(2 \pi)^{3}} n_{2}\left(n_{2}+1\right) .
$$

In the situation where the gluon 2 scattering elastically into gluon $k$ as shown in Figure E.19, with $\left(k \cdot k_{4}\right) \rightarrow 0$, the measured gluon is not involved in the collinear $1 \rightarrow 2$ branching which can be integrated fully. Is is straightforward to verify that this contribution yields a mild logarithmic divergence and hence can be neglected.

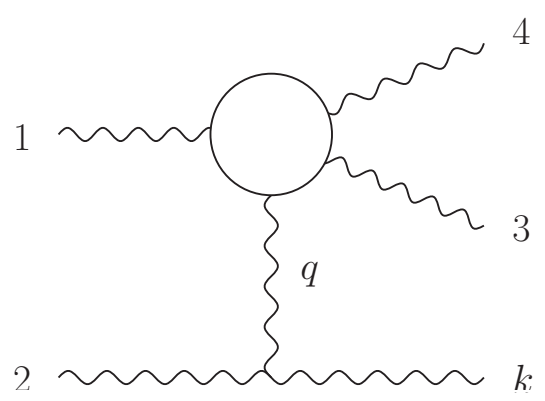

(a)

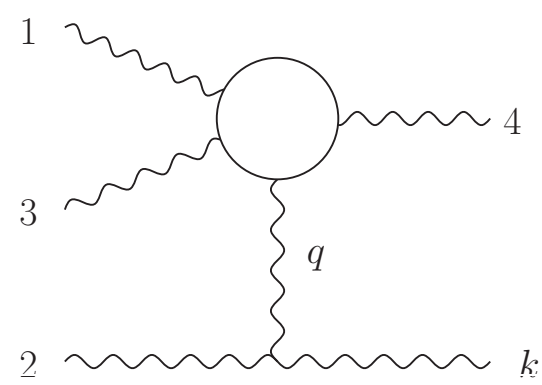

(b)

Figure E.19: Diagrammatic representation of the subdominant contribution to the 2 to 3 (a) and 3 to 2 (b) processes shown in Figure E.17, that corresponds effectively to the NLO correction to the elastic rate for small momentum transfer $q$.

\section{Appendix F. Effective 1 to 2 process}

The probability for a quasi-collinear branching of gluon with energy $\omega$ into two, with energies $z \omega$ and $(1-z) \omega$ respectively, has been calculated in the context of in-medium radiative parton energy loss. This result in the branching rate (4.7). In this appendix, using Eq. (4.7), we will derive explicit expressions for the branching rate in the BH and LPM regimes, respectively, that we use in this paper.

\section{Appendix F.1. The Bethe-Heitler regime}

In this regime the branching time is smaller that the typical elastic mean free path such that at most one scattering participates to the radiating process. This regime can be viewed as the reduction of the inelastic 2 to 3 
process to an effective 1 to 2 process involving a single small angle scattering. The single scattering approximation amounts to expanding Eq. (4.9) to first order in $\mathcal{C}\left(\boldsymbol{q}_{\perp}\right)$. The zeroth order reads

$$
S_{0}\left(\boldsymbol{p}_{\perp}, \boldsymbol{p}_{\perp}^{\prime}, z, \tau\right)=\exp \left[-i \frac{\boldsymbol{p}_{\perp}^{2}+m_{z}^{2}}{2 z(1-z) \omega} \tau\right] \theta(\tau) \delta\left(\boldsymbol{p}_{\perp}-\boldsymbol{p}_{\perp}^{\prime}\right)
$$

but do not contribute to the branching kernel as it yields an imaginary result. For the first order we have

$$
\begin{aligned}
& S_{1}\left(\boldsymbol{p}_{\perp}, \boldsymbol{p}_{\perp}^{\prime}, z, \tau\right)=-\frac{i}{2} \int_{0}^{\tau} \mathrm{d} \tau^{\prime} \exp \left[-i \frac{\boldsymbol{p}_{\perp}^{2}+m_{z}^{2}}{2 z(1-z) \omega}\left(\tau-\tau^{\prime}\right)\right] \\
& {\left[\tilde{\mathcal{C}}\left(\left(\boldsymbol{p}-\boldsymbol{p}^{\prime}\right)_{\perp} /(1-z)\right)+\tilde{\mathcal{C}}\left(\left(\boldsymbol{p}-\boldsymbol{p}^{\prime}\right)_{\perp} / z\right)+\tilde{\mathcal{C}}\left(\left(\boldsymbol{p}-\boldsymbol{p}^{\prime}\right)_{\perp}\right)\right]} \\
& \quad \times \exp \left[-i \frac{\boldsymbol{p}_{\perp}^{\prime 2}+m_{z}^{2}}{2 z(1-z) \omega} \tau^{\prime}\right] . \\
& =z(1-z) \omega \frac{\mathrm{e}^{-i \frac{\boldsymbol{p}_{\perp}{ }^{2}+m_{z}^{2}}{2 z(1-z) \omega} \tau}-\mathrm{e}^{-i \frac{\boldsymbol{p}_{\perp}{ }^{\prime 2}{ }^{2}+m_{z}^{2}}{2(1-z) \omega} \tau}}{\boldsymbol{p}_{\perp}{ }^{2}-\boldsymbol{p}_{\perp}{ }^{\prime 2}} \\
& \times\left[\tilde{\mathcal{C}}\left(\left(\boldsymbol{p}-\boldsymbol{p}^{\prime}\right)_{\perp} /(1-z)\right)+\tilde{\mathcal{C}}\left(\left(\boldsymbol{p}-\boldsymbol{p}^{\prime}\right)_{\perp} / z\right)+\tilde{\mathcal{C}}\left(\left(\boldsymbol{p}-\boldsymbol{p}^{\prime}\right)_{\perp}\right)\right] .
\end{aligned}
$$

Integrating over $\tau$ from zero to $\infty$, we find

$$
\begin{aligned}
& \int_{0}^{\infty} \mathrm{d} \tau S_{1}\left(\boldsymbol{p}_{\perp}, \boldsymbol{p}_{\perp}^{\prime}, z, \tau\right)=\frac{2 z^{2}(1-z)^{2} \omega^{2}}{\left(\boldsymbol{p}_{\perp}^{2}+m_{z}^{2}\right)\left(\boldsymbol{p}_{\perp}^{\prime 2}+m_{z}^{2}\right)} \\
& \times\left[\tilde{\mathcal{C}}\left(\left(\boldsymbol{p}-\boldsymbol{p}^{\prime}\right)_{\perp} /(1-z)\right)+\tilde{\mathcal{C}}\left(\left(\boldsymbol{p}-\boldsymbol{p}^{\prime}\right)_{\perp} / z\right)+\tilde{\mathcal{C}}\left(\left(\boldsymbol{p}-\boldsymbol{p}^{\prime}\right)_{\perp}\right)\right] .
\end{aligned}
$$

Thus,

$$
\begin{aligned}
& \mathcal{K}(z)=4 \alpha_{s} P(z) \int_{\boldsymbol{p}_{\perp}, \boldsymbol{p}_{\perp}^{\prime}} \frac{\boldsymbol{p}_{\perp} \cdot \boldsymbol{p}_{\perp}^{\prime}}{\left(\boldsymbol{p}_{\perp}^{2}+m_{z}^{2}\right)\left(\boldsymbol{p}_{\perp}^{\prime 2}+m_{z}^{2}\right)} \\
& \times\left[\tilde{\mathcal{C}}\left(\left(\boldsymbol{p}-\boldsymbol{p}^{\prime}\right)_{\perp} /(1-z)\right)+\tilde{\mathcal{C}}\left(\left(\boldsymbol{p}-\boldsymbol{p}^{\prime}\right)_{\perp} / z\right)+\tilde{\mathcal{C}}\left(\left(\boldsymbol{p}-\boldsymbol{p}^{\prime}\right)_{\perp}\right)\right] .
\end{aligned}
$$

This result is in fact incomplete because it includes only interference diagrams. Including the missing contributions that correspond to splitting before and after the scattering amouts to making the substitution $\boldsymbol{p}_{\perp}, \boldsymbol{p}_{\perp}^{\prime} \rightarrow$ 
$\left(\boldsymbol{p}-\boldsymbol{p}^{\prime}\right)_{\perp}^{2} / 2$, and together with the change of variables $\boldsymbol{q}_{\perp} \equiv \boldsymbol{p}_{\perp}-\boldsymbol{p}_{\perp}{ }^{\prime}$ and $\boldsymbol{k}_{\perp} \equiv \boldsymbol{p}_{\perp}$, we obtain

$$
\begin{aligned}
& \mathcal{K}(z)=2 \alpha_{s} P(z) \int_{\boldsymbol{k}_{\perp}, \boldsymbol{q}_{\perp}} \frac{\boldsymbol{q}_{\perp}^{2}}{\left(\boldsymbol{k}_{\perp}{ }^{2}+m_{z}^{2}\right)\left((\boldsymbol{k}-\boldsymbol{q})_{\perp}^{2}+m_{z}^{2}\right)} \\
& \times\left[\mathcal{C}\left(\boldsymbol{q}_{\perp} /(1-z)\right)+\mathcal{C}\left(\boldsymbol{q}_{\perp} / z\right)+\mathcal{C}\left(\boldsymbol{q}_{\perp}\right)\right] .
\end{aligned}
$$

Note that the delta function in $\mathcal{C}$ does not contribute to the splitting kernel. In the limit of vanishing thermal masses we recover the divergent result obtained in the previous section Eq. (E.19).

Let us evaluate the integral in the limit $z \rightarrow 0$ ot $(z \rightarrow 1)$. Due to nonlocality of interactions these regions give the dominant contribution to the collision integral. For $z \sim 1$, the splitting kernel yields

$$
\mathcal{K}(z) \simeq 4 \alpha_{s} P(z) \int_{\boldsymbol{k}_{\perp}, \boldsymbol{q}_{\perp}} \frac{1}{\left(\boldsymbol{k}_{\perp}{ }^{2}+m^{2}\right)\left((\boldsymbol{k}-\boldsymbol{q})_{\perp}^{2}+m^{2}\right)\left(\boldsymbol{q}_{\perp}^{2}+2 m^{2}\right)},
$$

where we have used $m_{D}^{2}=2 m^{2}$. The two angular integrations can be performed as follows: one is trivial and yields a factor $2 \pi$, after the change of variables $\boldsymbol{l}_{\perp} \equiv(\boldsymbol{k}-\boldsymbol{q})_{\perp}$, we then have

$$
\begin{aligned}
& \int_{\boldsymbol{k}, \boldsymbol{l}} \frac{1}{\left(\boldsymbol{k}^{2}+m^{2}\right)\left(\boldsymbol{l}^{2}+m^{2}\right)\left[(\boldsymbol{k}-\boldsymbol{l})^{2}+2 m^{2}\right]} \\
& =\int \frac{2 \pi|\boldsymbol{k}| \mathrm{d}|\boldsymbol{k}||\boldsymbol{l}| \mathrm{d}|\boldsymbol{l}|}{(2 \pi)^{4}\left(\boldsymbol{k}^{2}+m^{2}\right)\left(\boldsymbol{l}^{2}+m^{2}\right)} \int_{0}^{2 \pi} \frac{\mathrm{d} \theta}{\boldsymbol{k}^{2}+\boldsymbol{l}^{2}+2 m^{2}-2|\boldsymbol{k}||\boldsymbol{l}| \cos \theta} .
\end{aligned}
$$

Now the integration over $\theta$ yields

$$
\int_{0}^{2 \pi} \frac{\mathrm{d} \theta}{\boldsymbol{k}^{2}+\boldsymbol{l}^{2}-2 m^{2}-2|\boldsymbol{k}||\boldsymbol{l}| \cos \theta}=\frac{2 \pi}{\sqrt{\left(\boldsymbol{k}^{2}+\boldsymbol{l}^{2}+2 m^{2}\right)^{2}-4 \boldsymbol{k}^{2} \boldsymbol{l}^{2}}}
$$

and the remaining double integration over $x \equiv \boldsymbol{l}^{2} / m^{2}$ and $y \equiv \boldsymbol{k}^{2} / m^{2}$, yields the constant

$$
C=\int_{0}^{\infty} \frac{\mathrm{d} x \mathrm{~d} y}{(x+1)(y+1) \sqrt{(x+y+2)^{2}-4 x y}} \simeq 1.83 .
$$

We finally obtain

$$
\mathcal{K}(z)=\frac{4 \alpha_{s} N_{c}^{2} g^{4} I_{a} C}{(4 \pi)^{2} m^{2}} P(z)=\frac{\pi \bar{\alpha}^{2} C T_{*}}{N_{c}} P(z) .
$$


Appendix F.2. The LPM regime

Let us turn now to the LPM regime. In this regime multiple soft scatterings contribute to the branching process. Hence, the typical transverse momentum is larger that the thermal mass and the result will be less sensitive to medium scales. Hence, typical momentum transfer is relatively large, $\boldsymbol{q}_{\perp} \gg m_{D}$, allowing us to expand $\tilde{\mathcal{C}}\left(\boldsymbol{u}_{\perp}\right)$ in Eq. (4.9) to quadratic order in $\boldsymbol{u}_{\perp}$, we have

$$
\tilde{\mathcal{C}}\left(\boldsymbol{u}_{\perp}\right)=\int_{\boldsymbol{q}_{\perp}} \mathcal{C}\left(\boldsymbol{q}_{\perp}\right)\left(1-\mathrm{e}^{i \boldsymbol{x}_{\perp} \cdot \boldsymbol{q}_{\perp}}\right) \simeq \frac{g^{4} N_{c}^{2} I_{a}}{4 \pi} \boldsymbol{u}_{\perp}^{2} \ln \frac{1}{m_{D}\left|\boldsymbol{u}_{\perp}\right|} \simeq \frac{1}{4} \hat{q} \boldsymbol{u}_{\perp}^{2}
$$

where we have absorbed the logarithm in the transport coefficient $\hat{q}$ that we shall treat as a constant. As a result, the integration over $\boldsymbol{x}_{\perp}$ and $\boldsymbol{y}_{\perp}$ are Gaussian and yield

$$
\begin{aligned}
& S\left(\boldsymbol{p}_{\perp}, \boldsymbol{p}_{\perp}^{\prime}, z, \tau\right)=\frac{2 \pi i}{z(1-z) \omega \Omega \sinh (\Omega \tau)} \\
& \times \exp \left[-i \frac{\left(\boldsymbol{p}+\boldsymbol{p}^{\prime}\right)_{\perp}^{2}}{4 z(1-z) \omega \Omega \operatorname{coth}(\Omega \tau / 2)}-i \frac{\left(\boldsymbol{p}-\boldsymbol{p}^{\prime}\right)_{\perp}^{2}}{4 z(1-z) \omega \Omega \operatorname{coth}(\Omega \tau / 2)}\right] .
\end{aligned}
$$

with

$$
\Omega \equiv \frac{1+i}{2} \sqrt{\frac{\hat{q}\left[1+z^{2}+(1-z)^{2}\right]}{z(1-z) \omega}} .
$$

Using Eq. (F.12) in Eq. (4.7) and integrating over $\tau$ and the transverse momenta $\boldsymbol{p}_{\perp}$ and $\boldsymbol{p}_{\perp}{ }^{\prime}$, we obtain the splitting kernel in the LPM regime,

$$
\mathcal{K}(z)=\frac{\alpha_{s} N_{c}}{\pi} P(z) \sqrt{\frac{\hat{q}}{\omega}} \frac{\left(1-z+z^{2}\right)^{5 / 2}}{z^{3 / 2}(1-z)^{3 / 2}} .
$$

\section{Appendix G. Solution of Eq. (4.49)}

Let $P$ be a four-vector such that $\omega \equiv|P|$. We have then

$$
\nabla_{P}^{2} n(|P|)=\frac{1}{\omega^{3}} \frac{\partial}{\partial \omega} \omega^{3} \frac{\partial}{\partial \omega} n(\omega)
$$


Using the Green's function method in 4-dimensions we can write the solution formally as

$$
n(\omega)=\int_{0}^{\infty} \mathrm{d} t^{\prime} \int \frac{\mathrm{d}^{4} P^{\prime}}{(2 \pi)^{4}} G\left(\left|P-P^{\prime}\right|, t-t^{\prime}\right) S\left(\left|P^{\prime}\right|\right) .
$$

where the retarded Green's function $G$ obeys the following equation,

$$
\left[\frac{\partial}{\partial t}-\frac{\hat{q}_{\text {inel }}}{4} \nabla_{P}^{2}\right] G\left(\left|P-P^{\prime}\right|, t-t^{\prime}\right)=(2 \pi)^{4} \delta^{(4)}\left(P-P^{\prime}\right) \delta\left(t-t^{\prime}\right) .
$$

whose solution reads

$$
G\left(\left|P-P^{\prime}\right|, t-t^{\prime}\right)=\frac{16 \pi^{2} \theta\left(t-t^{\prime}\right)}{\left[\hat{q}_{\text {inel }}\left(t-t^{\prime}\right)\right]^{2}} \exp \left[-\frac{\left(P-P^{\prime}\right)^{2}}{\hat{q}_{\text {inel }}\left(t-t^{\prime}\right)}\right]
$$

In the case of interest, $\omega=|P| \gg\left|P^{\prime}\right|=\omega_{f}$, that is away from the injection scale, one can neglect the dependence of the Green's on the momentum $P^{\prime}$, it is then straightforward to integrate over $P^{\prime}$,

$$
\begin{aligned}
n(\omega, t) & \simeq \int_{0}^{t} \mathrm{~d} t^{\prime} G\left(\omega, t-t^{\prime}\right) \int \frac{\mathrm{d}^{4} P^{\prime}}{(2 \pi)^{4}} S\left(\left|P^{\prime}\right|\right) . \\
& =\frac{1}{4} P_{f} \int_{0}^{t} \mathrm{~d} t^{\prime} G\left(\omega, t^{\prime}\right) .
\end{aligned}
$$

Making the change of variable $x=\omega^{2} / \hat{q}_{\text {inel }}\left(t-t^{\prime}\right)$, the last integral yields,

$$
\begin{aligned}
\int_{0}^{t} \mathrm{~d} t^{\prime} G\left(\omega, t^{\prime}\right) & =\frac{16 \pi^{2}}{\hat{q}_{\text {inel }} \omega^{2}} \int_{\omega^{2} / \hat{q}_{\text {inel }}}^{\infty} \mathrm{d} x \mathrm{e}^{-x} \\
& =\frac{16 \pi^{2}}{\hat{q}_{\text {inel }} \omega^{2}} \exp \left(-\frac{\omega^{2}}{\hat{q}_{\text {inel }} t}\right) .
\end{aligned}
$$

Finally, the spectrum reads

$$
n(\omega) \simeq \frac{4 \pi^{2} P_{f}}{\hat{q}_{\text {inel }} \omega^{2}} \exp \left[-\frac{\omega^{2}}{\hat{q}_{\text {inel }} t}\right] .
$$




\section{Appendix H. Numerical procedure}

In the numerical calculations, we use a logarithmic grid in order to have a larger density of points in the infrared where the distribution diverges as a power law, namely,

$$
\ln p(i)=-\ln \left(p_{\max } / p_{\min }\right) \frac{n-i}{n-1}+\ln p_{\max },
$$

where $i=1, n, n=2000, p_{\max } \equiv p(n)=10-1000$ and $p_{\min } \equiv p(1)=$ $10^{-7}-10^{-2}$.

The gluon spectrum at $\tau+\Delta \tau$ is computed as follows

$$
f(\tau+\Delta \tau)=f(\tau)+C[f] \Delta \tau,
$$

with $\Delta \tau \simeq 10^{-2}-10^{-5}$. We use the Runge-Kutta (RK4) method to integrate over $q$ in the inelastic collision integral $C[f]$. As for the elastic part $f_{\mathrm{el}}(\tau)$ we use to the Backward-Time-Centered-Space Method "BTCS" in order to stabilize the diffusion term.

\section{References}

[1] J. Berges, J. P. Blaizot and F. Gelis, "EMMI Rapid Reaction Task Force on 'Thermalization in Non-abelian Plasmas'," J. Phys. G 39 (2012) 085115 [arXiv:1203.2042 [hep-ph]].

[2] X. G. Huang and J. Liao, "Kinetic evolution of the glasma and thermalization in heavy ion collisions," Int. J. Mod. Phys. E 23, 1430003 (2014) [arXiv:1402.5578 [nucl-th]].

[3] K. Fukushima, arXiv:1603.02340 [nucl-th].

[4] A. Kurkela, arXiv:1601.03283 [hep-ph].

[5] S. Mrowczynski, Phys. Lett. B 214, 587 (1988) Erratum: [Phys. Lett. B 656, 273 (2007)].

[6] P. B. Arnold, J. Lenaghan and G. D. Moore, JHEP 0308, 002 (2003) [hep-ph/0307325].

[7] M. Strickland, Braz. J. Phys. 37, 762 (2007) [hep-ph/0611349]. 
[8] P. B. Arnold and G. D. Moore, Phys. Rev. D 73, 025006 (2006) [hep$\mathrm{ph} / 0509206]$.

[9] A. Ipp, A. Rebhan and M. Strickland, Phys. Rev. D 84, 056003 (2011) [arXiv:1012.0298 [hep-ph]].

[10] P. B. Arnold and G. D. Moore, Phys. Rev. D 73, 025013 (2006) [hep$\mathrm{ph} / 0509226]$.

[11] A. H. Mueller, A. I. Shoshi and S. M. H. Wong, Nucl. Phys. B 760, 145 (2007) [hep-ph/0607136].

[12] J. Berges, K. Boguslavski, S. Schlichting and R. Venugopalan, Phys. Rev. D 89, no. 7, 074011 (2014) [arXiv:1303.5650 [hep-ph]].

[13] R. Baier, A. H. Mueller, D. Schiff and D. T. Son, "Bottom-up' thermalization in heavy ion collisions," Phys. Lett. B 502, 51 (2001) [arXiv:hep$\mathrm{ph} / 0009237]$.

[14] A. Kurkela and G. D. Moore, JHEP 1112, 044 (2011) [arXiv:1107.5050 [hep-ph]].

[15] A. Kurkela and G. D. Moore, Phys. Rev. D 86, 056008 (2012) [arXiv:1207.1663 [hep-ph]].

[16] J. Berges, K. Boguslavski, S. Schlichting and R. Venugopalan, Phys. Rev. D 89, no. 11, 114007 (2014) [arXiv:1311.3005 [hep-ph]].

[17] V.E. Zakharov. Springer. V.S. L'vov, and G. Falkovich, Kolmogorov Spectra of Turbulence -Verlag, Berlin, 1992, Vol. 1, p. 264.

[18] S. Nazarenko. 2011. Springer. Wave Turbulence, Lecture Notes in Physics. Vol. 825.

[19] A. V. Kats, V. M. Kontorovich, S. S. Moiseev, and V. E. Novikov, Zh. Eksp. Teor. Fiz. 71, 171 (1976)

[20] V. Mathieu, A. H. Mueller and D. N. Triantafyllopoulos, Eur. Phys. J. C 74, 2873 (2014) [arXiv:1403.1184 [hep-ph]].

[21] P. B. Arnold, G. D. Moore and L. G. Yaffe, JHEP 0301, 030 (2003) [hep-ph/0209353]. 
[22] J. P. Blaizot and E. Iancu, Phys. Rept. 359, 355 (2002) [hep$\mathrm{ph} / 0101103]$.

[23] J. P. Blaizot, F. Gelis, J. F. Liao, L. McLerran and R. Venugopalan, Nucl. Phys. A 873, 68 (2012) [arXiv:1107.5296 [hep-ph]].

[24] Jason Laurie, Victor S. L'vov, Sergey Nazarenko, and Oleksii Rudenko Phys. Rev. B 81, 1045262010

[25] Dyachenko, A., Newell, A.C., Pushkarev, A., Zakharov, V.E.: Optical turbulence: weak turbulence, condensates and collapsing fragments in the nonlinear Schrodinger equation. Physica D 57(1?2), 96 (1992)

[26] R. Micha and I. I. Tkachev, Phys. Rev. D 70, 043538 (2004) [hep$\mathrm{ph} / 0403101]$.

[27] Zakharov, V.E., Sagdeev, R.Z.: Spectrum of acoustic turbulence. Soviet Physics: Doklady 15, 439 (1970) [28]

[28] J. P. Blaizot, J. Liao and Y. Mehtar-Tani, arXiv:1609.02580 [hep-ph].

[29] L.W. Nordheim, Proc. R. Soc. London Ser. A 119 (1928) 689.

[30] Colm Connaughton, Yves Pomeau, C. R. Physique 5 (2004) 91106

[31] E.M. Lifshitz and L.P. Pitaevskii. Pergamon. Physical Kinetics Press, New York, 1981).

[32] J. P. Blaizot, J. Liao and L. McLerran, Nucl. Phys. A 920, 58 (2013) [arXiv:1305.2119 [hep-ph]].

[33] R. Baier, Y. L. Dokshitzer, A. H. Mueller, S. Peigne and D. Schiff, "Radiative energy loss and $\mathrm{p}(\mathrm{T})$ broadening of high-energy partons in nuclei," Nucl. Phys. B 484 (1997) 265 [hep-ph/9608322].

[34] Y. Mehtar-Tani, J. G. Milhano and K. Tywoniuk, Int. J. Mod. Phys. A 28, 1340013 (2013) [arXiv:1302.2579 [hep-ph]].

[35] J. P. Blaizot and Y. Mehtar-Tani, Int. J. Mod. Phys. E 24, no. 11, 1530012 (2015) [arXiv:1503.05958 [hep-ph]]. 
[36] P. Aurenche, F. Gelis and H. Zaraket, JHEP 0205, 043 (2002) [hep$\mathrm{ph} / 0204146]$.

[37] J. P. Blaizot, F. Dominguez, E. Iancu and Y. Mehtar-Tani, JHEP 1301, 143 (2013) [arXiv:1209.4585 [hep-ph]].

[38] P. B. Arnold, Phys. Rev. D 79, 065025 (2009) [arXiv:0808.2767 [hep$\mathrm{ph}]]$.

[39] J. P. Blaizot, E. Iancu and Y. Mehtar-Tani, Phys. Rev. Lett. 111, 052001 (2013) [arXiv:1301.6102 [hep-ph]].

[40] J. P. Blaizot and Y. Mehtar-Tani, Annals Phys. 368, 148 (2016) [arXiv:1501.03443 [hep-ph]].

[41] Kraichnan, R.: J. Fluid Mech. 47, 525 (1971)

[42] F. A. Berends, R. Kleiss, P. De Causmaecker, R. Gastmans and T. T. Wu, Phys. Lett. B 103, 124 (1981).

[43] X. G. Huang and J. Liao, Phys. Rev. D 91, no. 11, 116012 (2015) [arXiv:1303.7214 [nucl-th]].

[44] R. Baier, Y. L. Dokshitzer, A. H. Mueller, S. Peigne and D. Schiff, Nucl. Phys. B 483, 291 (1997) [hep-ph/9607355].

[45] B. G. Zakharov, JETP Lett. 63, 952 (1996) [hep-ph/9607440]. 